\title{
Physics book: \\ CRYRING@ESR
}

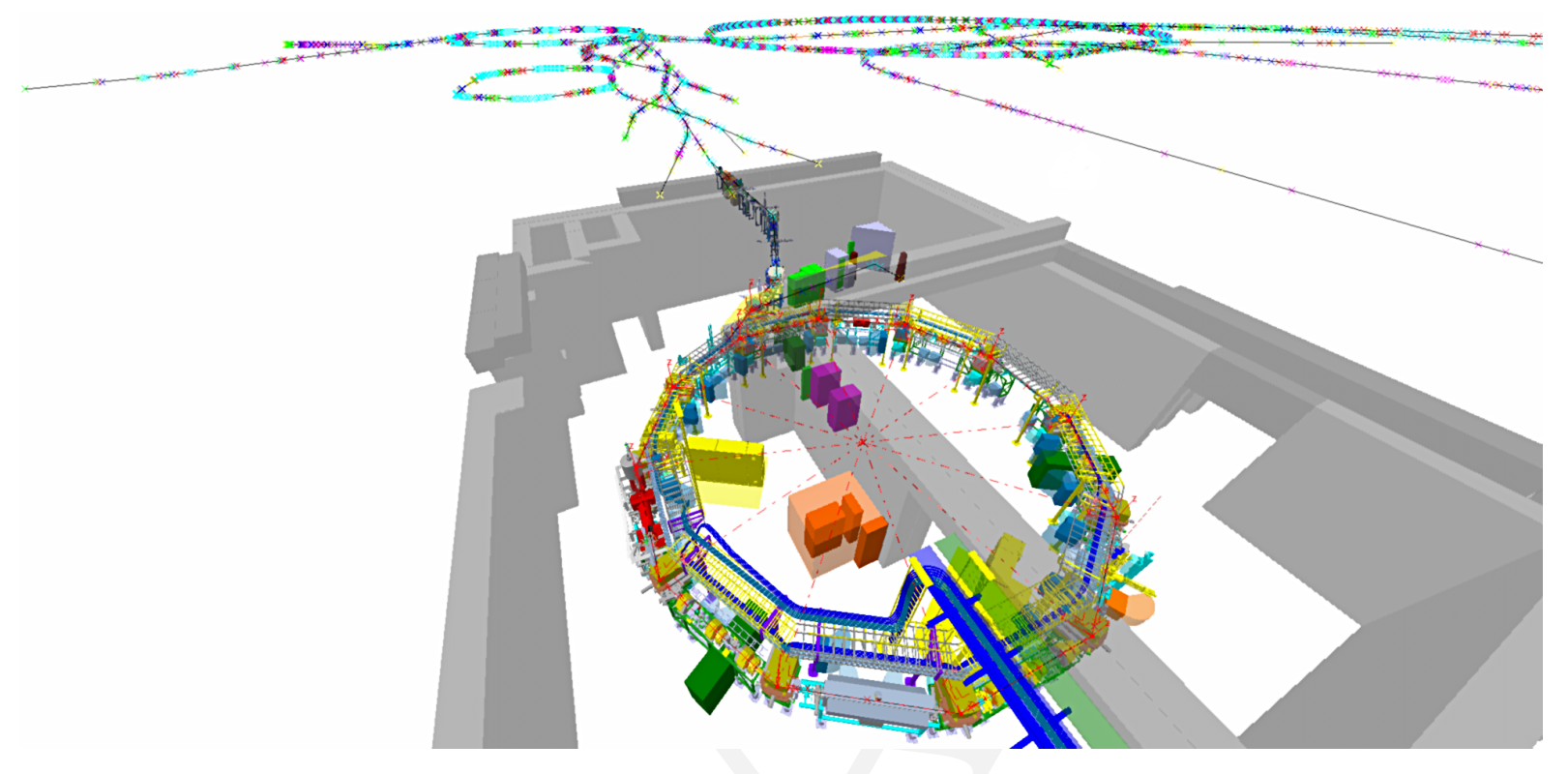

Editors:

M. Lestinsky, Y. Litvinov, Th. Stöhlker m.lestinsky@gsi.de

Atomic Physics Division

GSI Helmholtzzentrum für Schwerionenforschung

D-64291 Darmstadt

April 25, 2016

CRYRING ESR 


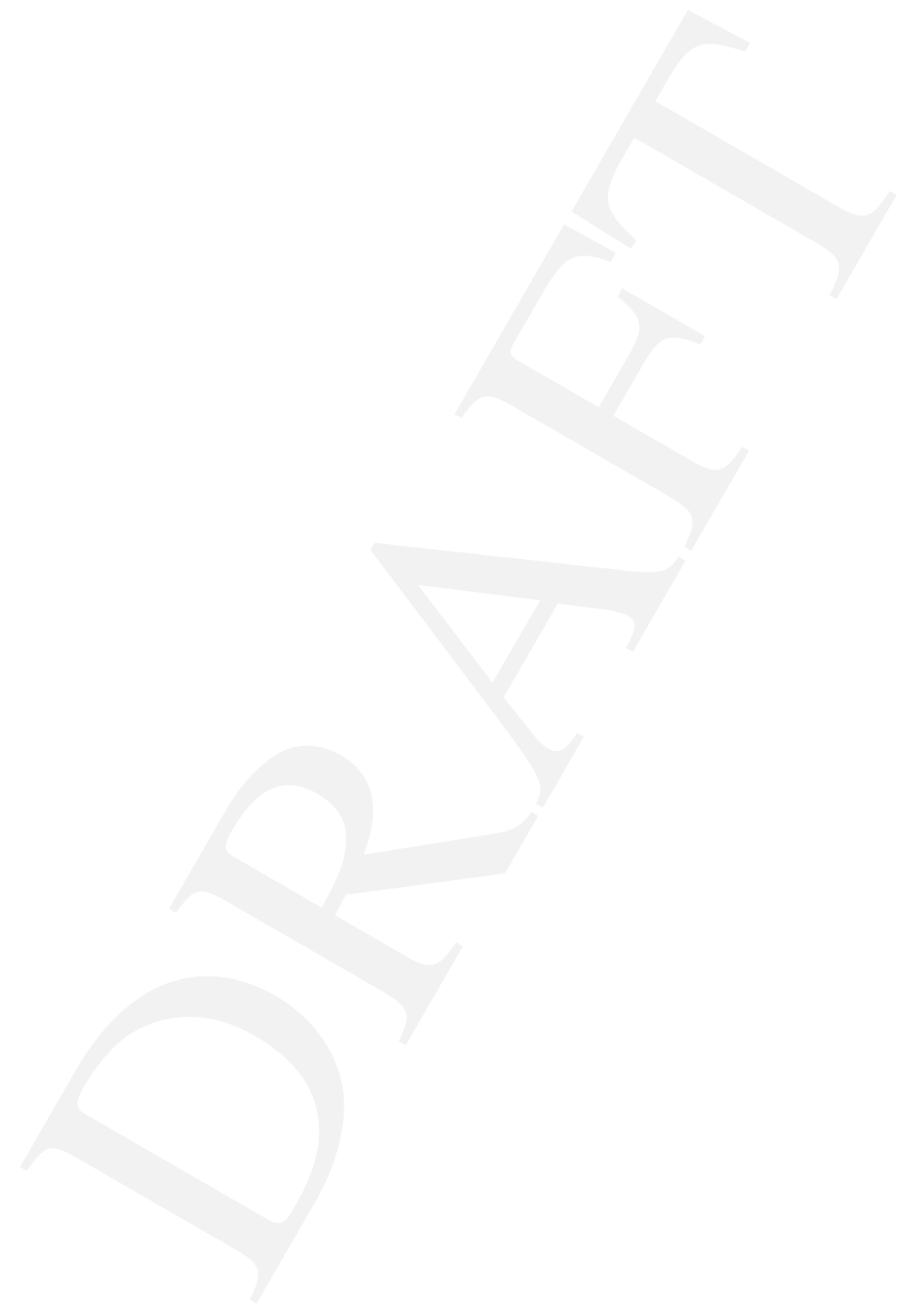




\title{
Physics book: CRYRING@ESR
}

\author{
Authors:
}

M. Lestinsky, ${ }^{1}$ V. Andrianov, ${ }^{1,2,3}$ B. Aurand, ${ }^{1}$ V. Bagnoud,${ }^{1}$ D. Bernhardt ${ }^{3}$ H. Beyer, ${ }^{1}$ S. Bishop, ${ }^{4}$ K. Blaum, ${ }^{5}$ A. Bleile, ${ }^{1,6}$ At. Borovik Jr. ${ }^{7}$ F. Bosch, ${ }^{1}$ C. J. Bostock, ${ }^{8}$ C. Brandau, ${ }^{1,7}$ A. Bräuning-Demian, ${ }^{1}$ I. Bray, ${ }^{8}$ T. Davinson, ${ }^{9}$ B. Ebinger, ${ }^{7}$ A. Echler, ${ }^{1,6,3}$ P. Egelhof, ${ }^{1,6}$ A. Ehresmann, ${ }^{10}$ M. Engström, ${ }^{11}$ C. Enss, ${ }^{12}$ N. Ferreira, ${ }^{5}$ D. Fischer, ${ }^{5}$ A. Fleischmann, ${ }^{12}$ E. Förster ${ }^{13,14}$ S. Fritzsche, ${ }^{13,14}$ R. Geithner, ${ }^{13}$ S. Geyer, ${ }^{16}$ J. Glorius ${ }^{16}{ }^{1}$ K. Göbel, ${ }^{16}$ O. Gorda, ${ }^{1}$ J. Goullon, ${ }^{5}$ P. Grabitz, ${ }^{1,6}$ R. Grisenti, ${ }^{1}$ A. Gumberidze, ${ }^{1}$ S. Hagmann, ${ }^{1}$ M. Heil,${ }^{1}$ A. Heinz ${ }^{21}$ F. Herfurth, ${ }^{1}$ R. Heß,,${ }^{1}$ P.-M. Hillenbrand, ${ }^{1}$ R. Hubele, ${ }^{5}$ P. Indelicato, ${ }^{15}$ A. Källberg, ${ }^{11}$ O. Kester, ${ }^{1,16}$ O. Kiselev, ${ }^{1}$ A. Knie, ${ }^{10}$ C. Kozhuharov, ${ }^{1}$ S. Kraft-Bermuth, ${ }^{3}$ T. Kühl, ${ }^{1}$ G. Lane, ${ }^{17}$ Yu. A. Litvinov, ${ }^{1},{ }^{5}$ D. Liesen, ${ }^{1}$ X. W. Ma, ${ }^{18}$ R. Märtin, ${ }^{14}$ R. Moshammer, ${ }^{5}$ A. Müller, ${ }^{3}$ S. Namba, ${ }^{19}$ P. Neumeyer ${ }^{20}$ T. Nilsson, ${ }^{21}$ W. Nörtershäuser, ${ }^{22}$ G. Paulus, ${ }^{13,14}$ N. Petridis, ${ }^{1}$ M. Reed, ${ }^{17}$

R. Reifarth, ${ }^{1,16}$ P. Reiß, ${ }^{10}$ J. Rothhardt, ${ }^{14}$ R. Sanchez, ${ }^{1}$ M. S. Sanjari, ${ }^{1}$ S. Schippers, ${ }^{7}$ H. T. Schmidt,,${ }^{11}$

D. Schneider, ${ }^{23}$ P. Scholz, ${ }^{3,7}$ R. Schuch, ${ }^{11}$ M. Schulz, ${ }^{24,16}$ V. Shabaev, ${ }^{25}$ A. Simonsson, ${ }^{11}$ J. Sjöholm, ${ }^{11}$ Ö. Skeppstedt, ${ }^{11}$ K. Sonnabend, ${ }^{1,16}$ U. Spillmann, ${ }^{1}$ K. Stiebing, ${ }^{16}$ M. Steck, ${ }^{1}$ T. Stöhlker, ${ }^{1,13,14}$ A. Surzhykov, ${ }^{26}$ S. Torilov, ${ }^{25}$ E. Träbert,${ }^{27} \mathrm{M}$. Trassinelli, ${ }^{28}$ S. Trotsenko, ${ }^{1,14} \mathrm{X}$. L. Tu, ${ }^{1,18} \mathrm{I}$. Uschmann, ${ }^{13,14}$ P. M. Walker, ${ }^{29}$ G. Weber, ${ }^{1,14}$ D. F. A. Winters, ${ }^{1}$ P. J. Woods, ${ }^{9}$ H. Y. Zhao, ${ }^{18}$ Y. H. Zhang, ${ }^{18}$ for the CRYRING@ESR Research Community

${ }^{1}$ GSI Helmholtzzentrum für Schwerionenforschung, 64291 Darmstadt, Germany

2 Institute of Nuclear Physics, Lomonosov Moscow State University, Moscow, Russia

${ }^{3}$ Institut für Atom- und Molekülphysik, Justus-Liebig-Universität, 35392 Gießen, Germany

${ }^{4}$ Physik Department E12, Technische Universität München, 85748 Garching, Germany

${ }^{5}$ Max-Planck-Institut für Kernphysik, 69117 Heidelberg, Germany

${ }^{6}$ Institute for Physics, Johannes Gutenberg University, Mainz, Germany

${ }^{7}$ I. Physikalisches Institut, Justus-Liebig-Universität, 35392 Gießen, Germany

${ }^{8}$ Institute of Theoretical Physics, Curtin University of Technology, Perth, Western Australia

${ }^{9}$ School of Physics and Astronomy, University of Edinburgh, Edinburg EH9 3JZ, UK

${ }^{10}$ Institute of Physics and CINSaT, University of Kassel, 34132 Kassel, Germany

${ }^{11}$ Fysikum, Stockholm University, SE-106 91 Stockholm, Sweden

12 Kirchhoff-Institut für Physik, Universität Heidelberg, D-69120 Heidelberg, Germany

${ }^{13}$ Friedrich Schiller Universität Jena, 07737 Jena, Germany

14 Helmholtz-Institut Jena, 07743 Jena, Germany

${ }^{15}$ Laboratoire Kastler Brossel, École Normale Supérieure, CNRS, Université Pierre et Marie Curie-Paris 6, F-75252 Paris CEDEX 05, France

16 Johann-Wolfgang-Goethe-Universität, 60438 Frankfurt am Main, Germany

17 Department of Nuclear Physics, RSPE, Australian National University, Acton, ACT 2601, Australia

18 Institute of Modern Physics, Chinese Academy of sciences, Lanzhou 730000, China

19 Graduate School of Engineering, Hiroshima University, Hiroshima 739-8527, Japan

${ }^{20}$ ExtreMe Matter Institut EMMI, 64291 Darmstadt, Germany

${ }^{21}$ Department of Physics, Chalmers University of Technology, SE-412 96 Gothenburg, Sweden

${ }^{22}$ Institut für Kernphysik, Universität Darmstadt, 64289 Darmstadt, Germany

${ }^{23}$ Lawrence Livermore National Laboratory, Livermore, CA 94551, USA

${ }^{24}$ Physics dept. and LAMOR, Missouri University of Science \& Technology, Rolla, MO 65409, USA

${ }^{25}$ Department of Physics, Sankt Petersburg State University, St. Petersburg 198504, Russia

${ }^{26}$ Physikalisches Institut, Universität Heidelberg, 69120 Heidelberg, Germany

27 Astronomisches Institut, Ruhr-Universität Bochum, 44780 Bochum, Germany

28 Inst. des NanoSciences de Paris, CNRS UMR7588 and UMPC-Paris 6, F-75015 Paris, France

${ }^{29}$ Department of Physics, University of Surrey, Guildford, GU2 7XH, UK 


\section{Contents}

1 Introduction

2 Atomic Physics $\quad 7$

2.1 Atomic Structure of highly charged ions $\ldots \ldots \ldots \ldots \ldots$

2.1.1 Precision X-ray Spectroscopy at CRYRING@ESR . . . . . . . . . . . . . . 7

2.1.2 Towards supercritical fields . . . . . . . . . . . . . . . . . . . . . 11

2.1.3 Atomic structure: electron-ion collisions . . . . . . . . . . . . . . . . 13

2.1 .4 Atomic lifetimes . . . . . . . . . . . . . . . . . . . 18

2.1.5 Polarization spectroscopy of hyperfine transitions in helium-like ions: A novel approach to study nuclear magnetic moments . . . . . . . . . . . . 20

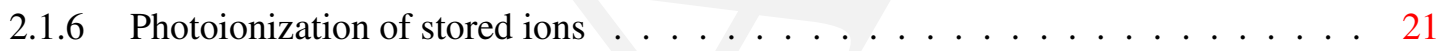

2.2 Atomic collisions dynamics . . . . . . . . . . . . . . . . . . . 25

2.2.1 Magnetic interaction and retardation effects upon the electron emission from HCI 25

2.2.2 Inelastic Electron Scattering in Inverse Kinematics. . . . . . . . . . . . . . 26

2.2.3 RECC and the high-energy endpoint of the $e$-nucleus bremsstrahlung . . . . . 29

2.2.4 Complete Atomic Fragmentation with Topologically Stable Multi-electron Transfer into the Projectile Continuum . . . . . . . . . . . . . . . . . . . 31

2.2.5 Ion-Lithium collisions with a MOTReMi in CRYRING . . . . . . . . . . . . . . 32

2.2.6 Two-center interference in charge transfer at $\mathrm{MeV}$ ion-molecule collisions . . . . 36

2.2.7 Collision of highly-charged ions with a hot, dense plasma . . . . . . . . . 37

2.2.8 Laser-induced recombination: Inverse above threshold ionization . . . . . . . . 39

3 At the borderline of atomic and nuclear physics 41

3.1 Accessing nuclear properties of stable nuclei and radio-isotopes with DR . . . . . . . . . 41

3.2 Search for the NEEC process . . . . . . . . . . . . . . . . . . 43

3.3 Laser-induced polarization of ions in storage rings . . . . . . . . . . . . . . . . 45

3.4 Combining laser spectroscopy with dielectronic recombination $\ldots \ldots \ldots$. . . . . . 47

4 Nuclear physics 49

4.1 Nuclear Structure and Nuclear Reactions . . . . . . . . . . . . . . . . . . . . . . . . . 49

4.2 Reaction measurements for nuclear astrophysics . . . . . . . . . . . . . . . 50

4.3 Direct measurements of nova $(p, \gamma)$ resonant reaction rates $\ldots \ldots \ldots \ldots \ldots$ 
5 Instrumentation and new techniques

5.1 Target section . . . . . . . . . . . . . . . . . . . . . . 59

5.2 Development of a VUV-VIS-Spectrometer for Target Characterisation . . . . . . . . . . 61

5.3 Micro-calorimeters for $\mathrm{x}$-ray spectroscopy . . . . . . . . . . . . . . . . . 64

5.3.1 Silicon micro-calorimeters $-\mathrm{SiM}-\mathrm{X} \ldots \ldots \ldots \ldots$. . . . . . . . . . 64

5.3.2 Metallic magnetic calorimeters $-\operatorname{maXs} \ldots \ldots \ldots 66$

5.4 A free-electron target in transverse collision geometry . . . . . . . . . . . 68

5.5 Detector for nuclear reactions at an internal gas-jet target $\ldots \ldots \ldots \ldots$. . . . . . 70

5.6 The Cryogenic Current Comparator for CRYRING@ESR . . . . . . . . . . . . . . . 71

5.7 Laser-based velocimetry and high-voltage measurements . . . . . . . . . . . . . 73

5.8 FLSR - the electrostatic Frankfurt low energy storage ring $\ldots \ldots \ldots \ldots$

6 Summary and Outlook $\quad 77$

$\begin{array}{ll}\text { Acronyms } & 81\end{array}$

$\begin{array}{lr}\text { List of Tables } & 85\end{array}$

$\begin{array}{lr}\text { List of Figures } & 87\end{array}$

$\begin{array}{lr}\text { Bibliography } & 89\end{array}$ 


\section{Chapter 1}

\section{Introduction}

\$Id: proj_description.tex,v 1.57 2016/04/25 11:19:31 lestinsk Exp \$

The exploration of the unique properties of stored and cooled beams of highly-charge ions as provided by heavy-ion storage rings has opened novel and fascinating research opportunities in the realm of atomic and nuclear physics research. Since the late 1980s, pioneering work has been performed at the CRYRING at Stockholm (Abrahamsson et al., 1993) and at the Test Storage Ring (TSR) at Heidelberg (Baumann et al., 1988). For the heaviest ions in the highest charge-states, a real quantum jump was achieved in the early 1990s by the commissioning of the Experimental Storage Ring (ESR) at GSI Helmholtzzentrum für Schwerionenforschung (GSI) in Darmstadt (Franzke, 1987) where challenging experiments on the electron dynamics in the strong field regime as well as nuclear physics studies on exotic nuclei and at the borderline to atomic physics were performed. Meanwhile also at Lanzhou a heavy-ion storage ring has been taken in operation, exploiting the unique research opportunities in particular for medium-heavy ions and exotic nuclei (Xia et al., 2002).

As described in the present report, the combination of ESR and CRYRING along with the availability of heavy bare, one- and few-electron ions and exotic nuclei will open a new horizon for research in the realm of matter under extreme condition.

Still today, ESR is the only storage ring worldwide which provides access to all naturally occurring elements up to the most extreme case of fully stripped uranium. Charge states and energies ( $4 \mathrm{MeV} / \mathrm{u}$ to $500 \mathrm{MeV} / \mathrm{u}$ ) can be tailored according to the requirements of the experiment (Eichler and Stöhlker, 2007). Challenging experiments in the realm of electron dynamics in extreme electromagnetic fields could be performed exploiting the brilliant, electron-cooled beams of the heaviest one- and few-electron ions such as, e.g., the $1 s$ Lamb shift, the hyperfine-splitting, or the investigation of dielectronic recombination. In nuclear physics and at the borderline to atomic physics, even direct mass measurements of short-lived radionuclides and the study of rare nuclear decay modes such as the bound-state $\beta$-decay or the orbital electron capture decay could be addressed for the very first time (Jung et al., 1992; Bosch et al., 1996; Kienle et al., 2013). Moreover, intense beams of highly charged in-flight synthesized radioisotopes are at the experimentalists disposition. One may note that this tremendous success of ESR in pushing the frontiers of physics into before inaccessible regimes was the reason why heavy-ion storage-rings are an essential pillar of the world's largest heavy-ion project, the international Facility for Antiproton and Ion Research (FAIR) project, currently under construction at Darmstadt. Likewise, CRYRING was operated at the Manne-Siegbahn-Laboratory in Stockholm with great success (Schuch et al., 2005; Schmidt et al., 2002, 2008; Misra et al., 2009; Vigren et al., 2009; Vigren et al., 2010; Orban et al., 2010), however with 
much lighter ions or heavy ions of lower charge state.

CRYRING is optimized for operation in an ion energy range of $\sim 14 \mathrm{MeV} / \mathrm{u}$ down to $\lesssim 300 \mathrm{keV} / \mathrm{u}$ or even lower energies, where the latter is mainly determined by the beam losses. Both rings, ESR and CRYRING operate in complementary ion energy ranges, and CRYRING thus bridges the gap between the lowest stable operation of ESR $(\approx 4 \mathrm{MeV} / \mathrm{u})$ and the HITRAP facility (Kluge et al., 2008; Herfurth et al., 2015) with highly-charged ions close to or even at rest whereby preserving the high luminosity, characteristic for stored ion beams. Moreover, for the low energy regime below $10 \mathrm{MeV} / \mathrm{u}$, the properties of CRYRING along with its instrumentation have several advantages compared to ESR such as its compactness, ultracold electron cooler, and the excellent ultrahigh vacuum conditions of $<10^{-11}$ mbar. By combining ESR and CRYRING, the entire energy range from $500 \mathrm{MeV} / \mathrm{u}$ almost down to rest will be available for experiments dealing with intense beams of highly charged ions and exotic nuclei.CRYRING@ESR will be the only facility world-wide that provides low-energy highly charged stable beams and beams of rare isotopes with a free choice of the charge state, including bare ions. Radioisotopes synthesized in a target after Schwerionensynchrotron (SIS18) can be purified from unwanted contaminants either with the present Fragment Separator (FRS) (Geissel et al., 1992) or alternatively directly in the ESR by employing its high resolving power (Steck et al., 2010; Brandau et al., 2013). Of particular scientific interest are stable ions and artificially synthesized nuclides dressed with few electrons or fully stripped. In such elementary ionic systems the physics topic under investigation is not masked or hampered by many-body effects. Moreover, low-energy phase space of cooled ions provide extremely well-defined experimental conditions for high precision spectroscopy experiments (electrons, ions, and photons). At the same time, compared to ions at rest, orders of magnitude higher luminosity in collision experiments using low-energy ions in a storage ring is achieved due to the repeated interaction of the stored ion beam with a target (gas, free electrons, laser). For atomic collision studies, another appealing aspect is the very strong perturbation to the target atoms due to the impact of the slow highly charged ions with conditions far from equilibrium. In combination with high-luminosities, even the study of heavy quasi-molecules might get accessible for the very first time when heavy highly-charged ions interact with a heavy atomic target, a real terra-incognita. In addition, CRYRING operates in a particularly interesting energy range for nuclear reactions, that is at the Coulomb barrier and in the astrophysically relevant Gamow window of $p$ - and $r p$-processes of nucleosynthesis. The swift low-energy beam also makes the detection and monitoring of primary and secondary ions either in a destructive manner with particle detectors or in a non-destructive manner down to the level of single particles by means of Schottky techniques experimentally much easier. The dynamic range that is covered with the various experimental techniques covers the span from single stored ions, detected by means of Schottky noise analysis up to beams at the space charge limit.

Finally we like to stress the important role of CRYRING@ESR for the portfolio of storage-ring and trapping facilities of the future FAIR facility (see Figure 1.1). This unrivaled combination of storage and trapping facilities is a unique feature of FAIR that distinguishes FAIR from all other planned or operating particle accelerators worldwide. These FAIR facilities cover a beam-energy range of more than 10 orders of magnitude, enabling precision experiments with brilliant and cooled ion beams starting at beam energies of as high as $5 \mathrm{GeV} / \mathrm{u}$ down to basically rest in the laboratory. Consequently, already with the installation of CRYRING@ESR and together with HITRAP, an important part of the heavy ion program as foreseen at FAIR can already be addressed with the synchrotron SIS18, and can been exploited by the various storage-ring collaborations of the international FAIR project with their dedicated 
physics programs (see the related FAIR documents SPARC, 2005; EXL, 2006; ILIMA, 2006). One may note, that even the challenging program of the Facility for Low-energy Antiproton and Ion Research (FLAIR) collaboration might get realized by the CRYRING@ESR facility once antiprotons at FAIR will be available, provided a dedicated transfer beamline from the antiproton-production facility of FAIR to the CRYRING becomes available (Stöhlker et al., 2014; Widmann, 2015; Katayama et al., 2015).

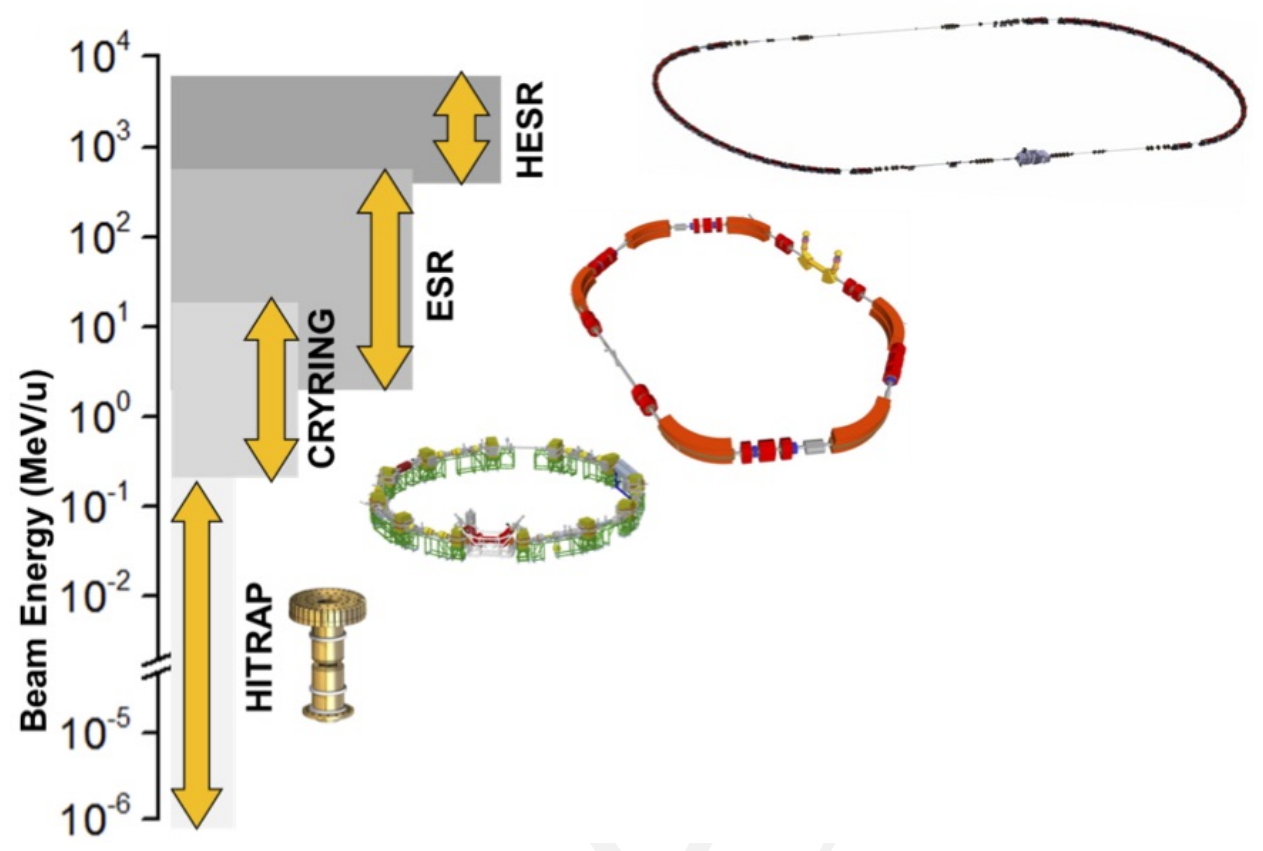

Figure 1.1. Storage facilities at GSI and FAIR compared to their respective energy ranges for highly-charged ions. Adopted from Stöhlker et al. (2015).

We briefly outline the present parameters of CRYRING at the ESR, such as they will be relevant for the discussion of the proposed experiment program. A full technical description of the original CRYRING setup in its original form is given by Danared et al. (2011). The CRYRING@ESR-project required to make some amendments, these have been worked out in Lestinsky et al. (2012). The project is currently under construction and a recent progress report is given by (Lestinsky et al., 2015). Table 1.1 summarizes the expected operation parameters of CRYRING@ESR and Figure 1.2 illustrates the location within a modified GSI storage ring topology. A closer view of the new CRYRING installations may be seen in Figure 1.3. The ring has a diameter of about $18 \mathrm{~m}$ and the circumference on the central orbit is $54.17 \mathrm{~m}$. Its new circumference is slightly increased over the former Stockholm configuration by $\approx 2.5 \mathrm{~m}$ and now matches half the orbit length ESRs extraction orbit. This additional length is evenly distributed to the 'non-magnetic' straight sections. Stored ions are bent into a closed orbit using twelve $30^{\circ}$ dipole magnets connected by twelve straight sections. The ring has sixfold supersymmetry, meaning that the pattern of ion optical elements repeats every second section. Beam focussing is achieved with quadrupole magnets and sextupoles correct for chromaticity. In between each two focussing section are interleaved 'low dispersion' sections, where injection, electron cooling, RF de- and acceleration, extraction, a free straight section for targets, and a diagnostics section are implemented. Ions can be stored at rigidities $B \rho$ between $0.054 \mathrm{Tm}$ and $1.44 \mathrm{Tm}$. CRYRING is capable of fast ramping of the ion beam energy at slew rates of $1 \mathrm{~T} / \mathrm{s}$ or $7 \mathrm{~T} / \mathrm{s}$, which corresponds approximately to $1 \mathrm{~s}$ or $150 \mathrm{~ms}$ ramping times, respectively, through the full dynamic range of achievable ion energies in CRYRING, thus short-lived 
Table 1.1. Operation parameters of CRYRING@ESR with heavy ions from SIS18/ESR facility (collated from Danared et al., 2011; Lestinsky et al., 2012, 2015).

\begin{tabular}{ll}
\hline Circumference & $54.17 \mathrm{~m}$ \\
Bending radius in $30^{\circ}$ dipole magnets & $1.2 \mathrm{~m}$ \\
Rigidity at injection & \\
$\quad$ - for $p, \bar{p}$ & $0.80 \mathrm{Tm}$ \\
$\quad$ - for heavy ions & $1.44 \mathrm{Tm}$ \\
Highest possible injection energy & \\
$\quad$ - for $p, \bar{p}$ & $30 \mathrm{MeV}$ (capped by radiation safety) \\
$\quad$ - for ${ }^{12} \mathrm{C}^{6+}$ & $24.7 \mathrm{MeV} / \mathrm{u}$ \\
$\quad$ - for ${ }^{238} \mathrm{U}^{92+}$ & $14.8 \mathrm{MeV} / \mathrm{u}$ \\
Lowest rigidity & $0.054 \mathrm{Tm}$ \\
Lowest energy & charge exchange limited \\
Ion beam lifetimes & $3 \mathrm{~s}-15$ min \\
Magnet ramping & $1 \mathrm{~T} / \mathrm{s}(\mathrm{upgrade}$ to $7 \mathrm{~T} / \mathrm{s}$ possible) \\
Vacuum pressure ( $\mathrm{N}_{2}$ equiv.) & $10^{-11}-10^{-12} \mathrm{mbar}$ \\
Beam injection & multi-turn and fast \\
Beam extraction & slow and fast \\
Local injector beamline & $40 \mathrm{keV} / q ; 300 \mathrm{keV} / u$ for $m / q \leq 2.85$ \\
Transition energy, $\gamma_{\mathrm{tr}}$ & $2.30 @ Q_{x}=2.42$ \\
\hline
\end{tabular}

species are within reach. The vacuum conditions of CRYRING are excellent with $\sim 10^{-12}$ mbar and long storage times of around 10 to $1000 \mathrm{~s}$ are expected, depending on ion energy and charge state. Besides ion injection from ESR, a local injector beamline is prepared, which allows for continued service even at times, where major shutdowns of the GSI accelerators is e.g. a necessity of FAIR construction. The local injector is equipped with a $300 \mathrm{keV} / \mathrm{u} \mathrm{RFQ}$ (for $m / q \leq 2.85$ ) and a limited reach of available ion species, depending on compatible ion sources.

In the following chapters we sketch out a broad scientific program in the fields of atomic and nuclear physics and at their intersection. The realization will allow for exciting high-precision spectroscopy studies of atomic systems and their dynamics where special emphasis is given to the effects of quantum electrodynamics (QED) and electron-correlation in the strong field domain (Chapter 2). Here, also the intersection of atomic and nuclear physics is addressed where the imprint of nuclear effects on the electronic shell are investigated with spectroscopic methods (Chapter 3), and exploring the nuclear structure, nuclear dynamical processes and quantitative measurements of astrophysically relevant $(p, \gamma)$-reaction rates (Chapter 4). These experiments are of prime interest for testing modern theoretical methods on fundamental processes as well as for applications in astrophysics and for modelling plasmas. In the domain of slow collisions in of heavy ions at highest charge-states where atomic processes are prevailed by large perturbations, these studies are expected to refine substantially our understanding of the physics of extreme electromagnetic fields. Also, CRYRING@ESR will offer extracted high-quality ion beams, thus enabling novel research opportunities for external-target experiments with slow highly-charged ions. The 


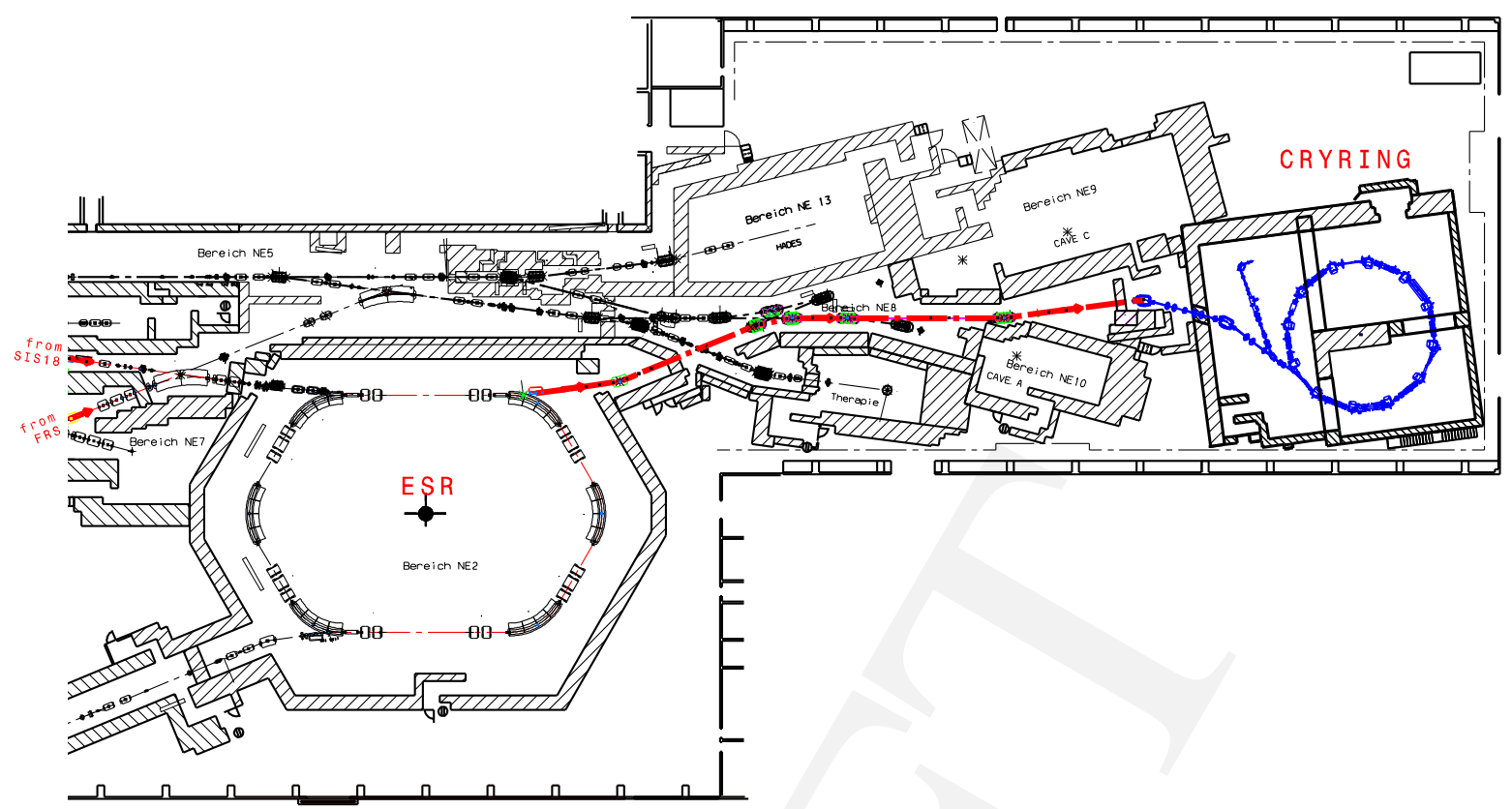

Figure 1.2. The new storage ring topology at GSI with CRYRING@ESR inside the former Cave B in the Target Hall of GSI. The CRYRING is shown with blue lines, the thick red line indicates the transfer beamline from ESR to CRYRING. A detailed view and discussion of the modified CRYRING cave is given in Lestinsky et al. (2012).

experimental boundary conditions and suggested future instrumentation upgrades are outlined in Chapter 5. We conclude this text in Chapter 6. This manuscript largely focusses on research that will be possible with CRYRING@ESR immediately after the project is commissioned and with ion beams that are already presently available utilizing the present GSI accelerator facilities and at ESR and downstream. 


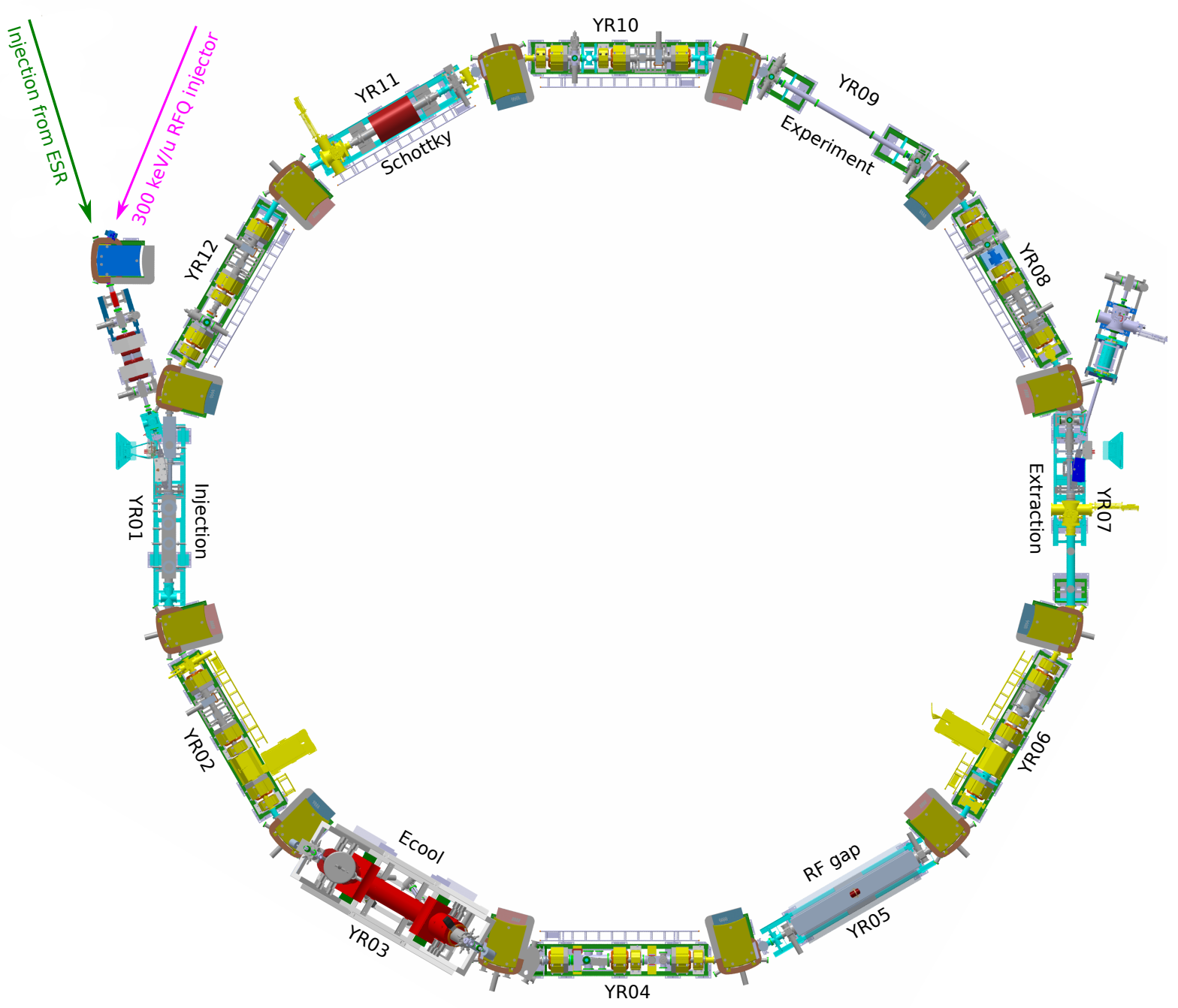

Figure 1.3. Top view of the CRYRING model in the new CRYRING@ESR configuration. Labels indicate the section numbering and the dominant functions of each straight section. Please see text for a further description. 


\section{Chapter 2}

\section{Atomic Physics}

\$Id: atomic_physics.tex,v 1.21 2016/04/08 12:50:49 lestinsk Exp \$

\subsection{Atomic Structure of highly charged ions}

\subsubsection{Precision X-ray Spectroscopy at CRYRING@ESR}

Storage rings for heavy ions provide unique conditions for $\mathrm{x}$-ray spectroscopy of photonic transitions involving the interaction of high- $Z$ few electron ions with particle probes, such as atoms in a gas target or with electron beams (Stöhlker et al., 1995, 1998; Rzadkiewicz et al., 2006; Gumberidze et al., 2010; Reuschl et al., 2008; Eichler and Stöhlker, 2007; Nofal et al., 2007). The high luminosity of storage rings along with very clean, almost background free experimental conditions are a prerequisite for the success of these studies where elementary and fundamental dynamical processes (Stöhlker et al., 1995; Gumberidze et al., 2010; Reuschl et al., 2008; Eichler and Stöhlker, 2007; Nofal et al., 2007; Banaś et al., 2015) as well as the structure properties of high- $Z$ one- and few-electron ions have been addressed in a broad range of experiments by applying various techniques, e.g. angular distribution studies (Stöhlker et al., 1997; Stöhlker et al., 1999, 2001), polarimetry (Tashenov et al., 2006, 2014; Weber et al., 2010; Märtin et al., 2012), and precision x-ray spectroscopy (Stöhlker et al., 1993; Beyer et al., 1995; Stöhlker et al., 2000; Gumberidze et al., 2005, 2004; Trassinelli et al., 2009; Beyer et al., 2004; Chatterjee et al., 2006; Trotsenko et al., 2010). By combining the ESR and CRYRING facilities, together with novel instrumentation as currently developed by Stored Particles Atomic Physics Research Collaboration (SPARC), a large discovery potential is expected to open up in such studies. Precision experiments with utmost accuracy can be anticipated as well as the exploration of new regimes for the study of atomic collision dynamics which have been inaccessible up to now. As an example, for the very first time, transverse electron targets (Section 5.4) will allow experimenters to study the details of the photo-recombination or electron-impact excitation processes in free-electron-ion collisions by measuring the polarization as well as the angular differential cross-sections by means of dedicated x-ray polarimeters (Tashenov et al., 2006; Weber et al., 2010; Märtin et al., 2012) and spectrometers (Beyer et al., 2004, 2015; Chatterjee et al., 2006).

In the following, we discuss two possible experiments at the CRYRING. The first one aims at a most accurate determination of the $1 s$ Lamb shift in high- $Z$ H-like ions. The goal of the second experiment is a high precision measurement of the electron-electron correlation in the ground state of the heaviest Helike systems. These are only prominent examples and the experimental arrangement discussed can easily 

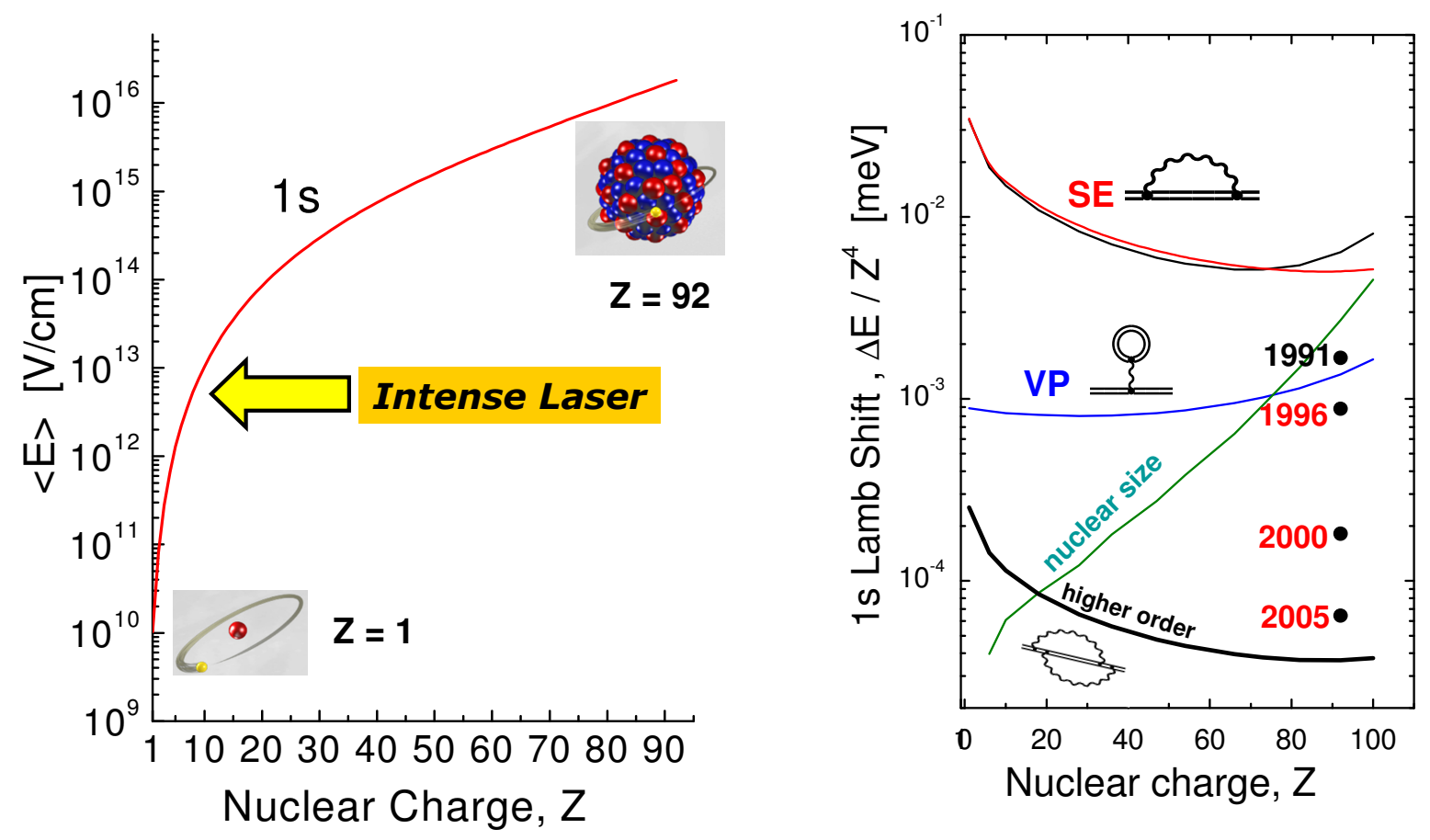

Figure 2.1. Left panel: expectation value of electric field strength for the $1 s$ ground state of hydrogen-like ions in the range of the nuclear charge $Z=1-92$ (Soff et al., 1998). Right panel: various contributions to the $1 s$ Lamb shift as functions of the nuclear charge in comparison to experimental precision achieved at the various experiments.

be transferred to the investigation of Li-like or other few-electron ions, to be conducted in experiments either at the electron cooler or at the internal target of CRYRING.

\section{The ground state Lamb shift in very heavy $\mathrm{H}$-like ions}

One of the frontiers of QED is the study of electrons in intense electromagnetic fields. As such, the $1 \mathrm{~s}$ Lamb shift in the heaviest (stable) one-electron ion available in the laboratory, $\mathrm{U}^{91+}$, poses as the ultimate test bed for precision experiments. In H-like uranium, the electric field strength is already close to the critical value for the spontaneous emission of electron-positron pairs which makes it ideal for testing the limits of our understanding of bound-state QED (see Figure 2.1). Despite the enormous success in the low-field domain (Niering et al., 2000; Beauvoir et al., 2000; Parthey et al., 2011; Matveev et al., 2013), there are still controversial results for exotic atoms (Pohl et al., 2010; Mohr et al., 2008; Antognini et al., 2013; Pohl et al., 2013; Jentschura, 2015; Karshenboim, 2015) and the non-perturbative regime at high $Z$ definitely remains still a challenge for both experiments and theory.

At the ESR, the topic of the $1 s$ Lamb shift has already attracted much attention by a series of experiments performed during the last decade. By exploiting the brilliant beams of stored and cooled ions with well-defined momentum and energy, the experimental accuracy could be improved considerably over the years (see Figure 2.1) and the study of the $1 s$-Lamb shift in high- $Z$ ions can nowadays be regarded as one of the most accurate tests of strong-field QED for one-electron systems. In these experiments, very different approaches have been applied (electron cooler, internal target etc.) and carefully analyzed with respect to their potential for future developments. This provides a solid basis for an anticipated future scenario to push the experimental accuracy to a limit which is necessary for accessing the higher-order 

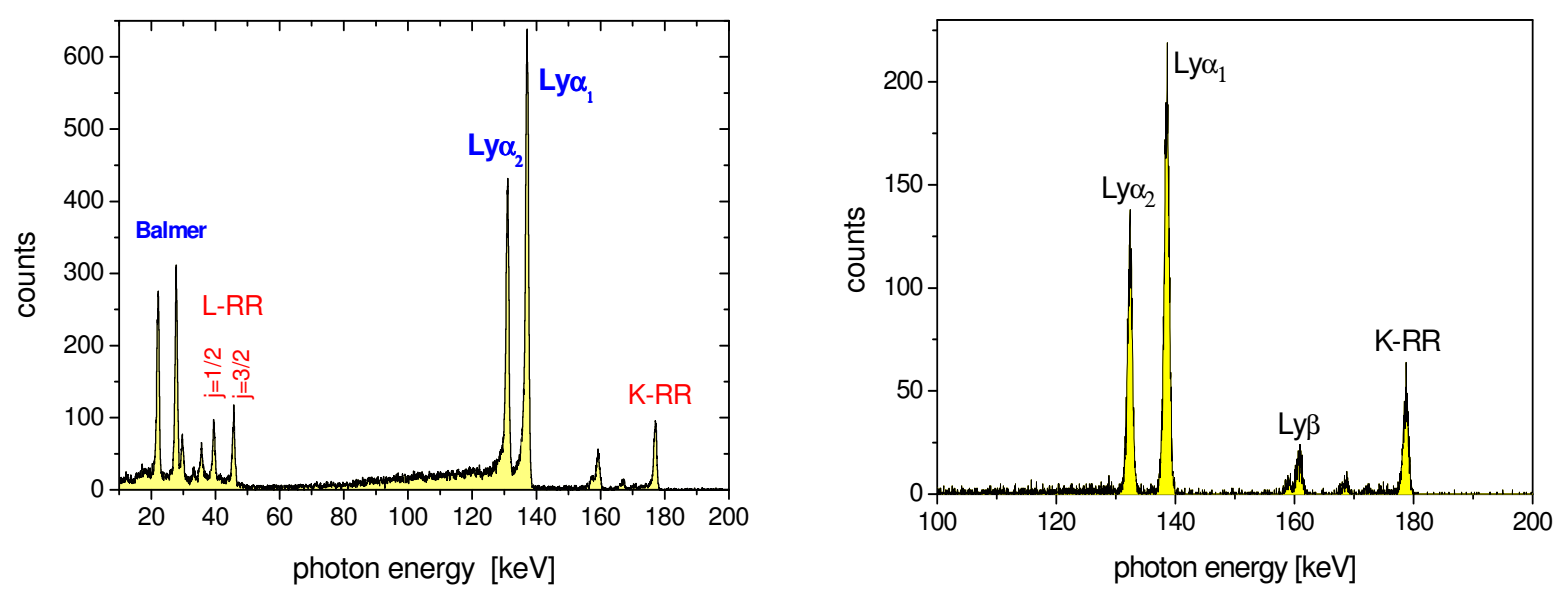

Figure 2.2. Left panel: X-ray spectrum (laboratory frame) following the radiative recombination of electrons with bare uranium ions as measured at the ESR electron cooler with an x-ray detector mounted at close to 0 degree observation angle with respect to the ion beam direction. The beam energy in this experiment amounted to $43.6 \mathrm{MeV} / \mathrm{u}$. Right panel: the same spectrum, but after subtraction of delayed coincidences caused by electron capture into Rydberg states (Reuschl et al., 2008; Gumberidze et al., 2005).

QED effects whose evaluation has been recently completed following very extensive theoretical work (Yerokhin and Shabaev, 2015). A highly desired experimental scenario would be quite similar to the experimental scheme which has been applied very successfully for the study of the $1 \mathrm{~s}$-Lamb Shift at the ESR electron cooler (Beyer et al., 1995; Gumberidze et al., 2005) (see also Figure 2.2) except that, besides $0^{\circ}$ spectroscopy, photon detection at $180^{\circ}$ (Banaś et al., 2015) will be added. Therefore, the new approach includes the combination of $0^{\circ}$ and $180^{\circ}$ spectroscopy at the electron cooler for strongly decelerated ions at energies close to $10 \mathrm{MeV} / \mathrm{u}$. Moreover, in contrast to the previous experiments where semiconductor x-ray detectors were utilized, here, novel high-resolution photon-detection techniques with a large wavelength acceptance will be applied. Furthermore, time coincidences between the photon spectrometers and a particle counter for down-charged ions would provide clean spectra and, therefore, are highly desirable. All of these conditions are expected to be fulfilled in an experiment at the electron cooler of the CRYRING by using novel micro-calorimeters (Egelhof et al., 1996; Bleile et al., 2000; Andrianov et al., 2008; Pies et al., 2012; Hengstler et al., 2015; Kraft-Bermuth et al., 2015), providing excellent energy resolution, timing capability and a reasonable detection efficiency for x-ray energies in the range of 20 to $150 \mathrm{keV}$ (see Figure 2.3). One may note that such detectors are part of the R\&D program of the SPARC collaboration. Such an experimental scheme applied at CRYRING provides several unique features:

1. At the low beam energies of the CRYRING the Doppler corrections and corresponding uncertainties are small. Moreover, as the ion velocity is determined by the electron energy in the electron cooler, which in turn can be measured with very high accuracy using a new dedicated voltage calibration device for the electron cooler (Andelkovic et al., 2015, chap. 9), the Doppler shift can be determined with very good accuracy. Both, the low ion energy and the precise measurement of the ion velocity, are particularly important, as the systematic uncertainty introduced by the velocity determination was one of the main limitations of the $1 s$ Lamb shift accuracy achieved in previous experiments at the ESR electron cooler. 


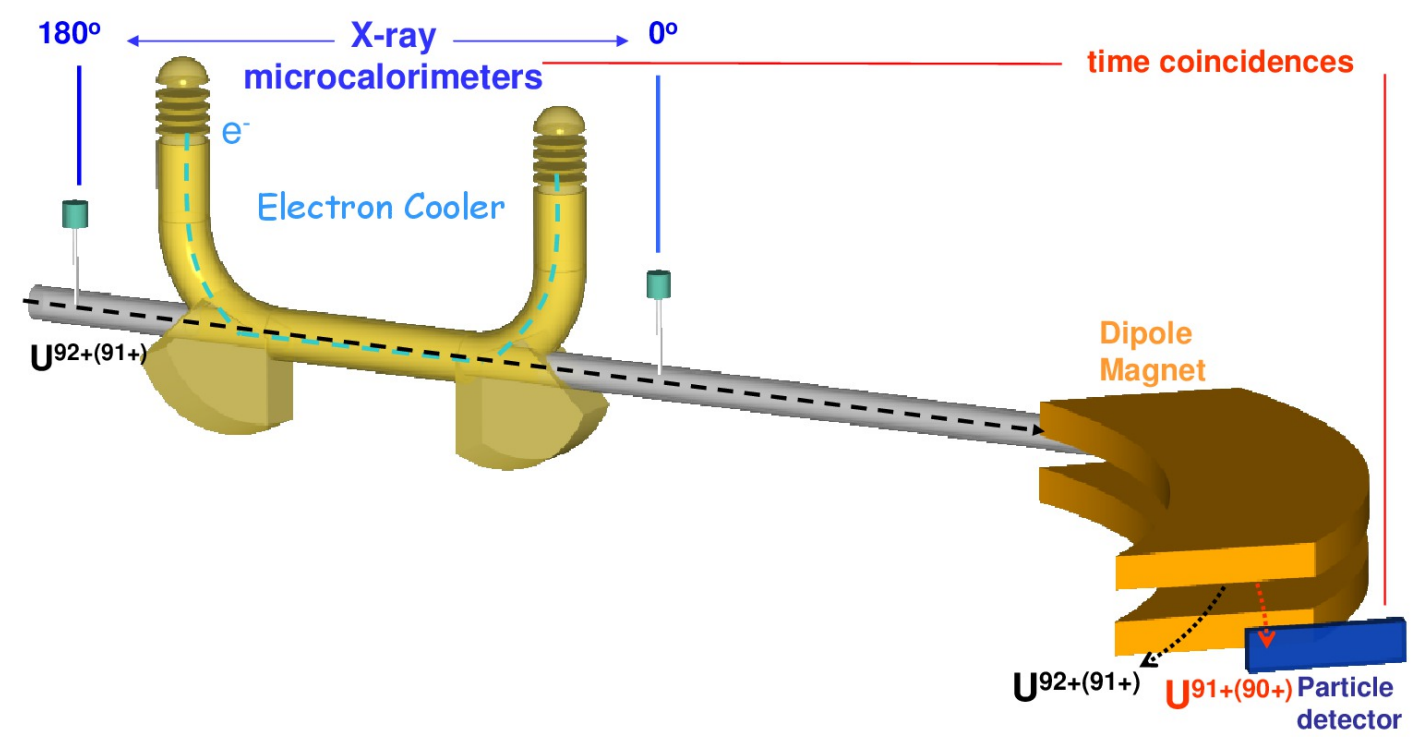

Figure 2.3. Scheme for a possible experimental setup at the electron-cooler of the CRYRING. X-rays will be observed at close to $0^{\circ}$ and $180^{\circ}$ with respect to the beam axis and recorded in coincidence with the down-charged ions $\left(\mathrm{U}^{91+}, \mathrm{U}^{90+}\right)$.

2. By combining 0- and 180-degree spectroscopy it is possible to eliminate Doppler corrections to a very large extent since only uncertainties in the determination of the ion velocity but not of the observation angle will contribute.

3. The large x-ray energy acceptance of the envisaged detection scheme will accommodate the measurement of radiative-recombination (RR) transitions into the $\mathrm{K}$ shell as well as into the $j$ sublevels of the L shell, and the subsequent Lyman and Balmer transitions all at the same time without changing detectors. Moreover, due to the excellent energy resolution even the RR transitions into the $2 s_{1 / 2}$ and $2 p_{1 / 2}$ as well as the $2 s_{1 / 2} \rightarrow 1 s_{1 / 2}$ and $2 p_{1 / 2} \rightarrow 1 s_{1 / 2}$ Lyman transitions can be expected to be energetically resolved. In return, this provides a high level of redundancy which facilitates the simultaneous determination of the $2 s$ together with the $1 s$ Lamb shift.

4. The geometrical boundary conditions at the electron cooler of the CRYRING provide more favorable conditions than those at the ESR electron cooler and, in particular, much larger solid angles become accessible: the effective cooler length at the CRYRING is $0.8 \mathrm{~m}$ compared to $2.5 \mathrm{~m}$ at the ESR; the distance between the detector and the center of the electron cooler is $2 \mathrm{~m}$ compared to $4.1 \mathrm{~m}$. Moreover, the envisaged multi-pixel detectors provide high granularity and, hence, high angular resolution of $\mathrm{x}$-ray observations.

An alternative approach for high precision measurement of the ground-state Lamb shift in high- $Z$ one-electron systems is based on the gas jet target of CRYRING. Here, the advantage will be a higher photon event rate due to higher (bound-)electron densities $n_{e}$ provided by the gas jet target as compared to the free-electron densities available at the electron cooler. In addition, there is the possibility to mount the photon detectors closer to the ion-target interaction zone. A disadvantage of a gas target compared to the scenario at the electron cooler will be that, in addition to uncertainties in the determination of the ion velocity, there will be uncertainties related to the photon observation angle. This, however, can be, at least partially, overcome by observing simultaneously the Lyman and the Balmer transitions. The Balmer 
series is very weakly influenced by QED effects and thus the associated line energies can be calculated with very high accuracy. Thus, the Balmer transitions would provide a means to determine the Doppler transformation factors independently from a measurement of the observation angle and the beam velocity. This will be possible due to the large energy-acceptance range of modern x-ray microcalorimeters.

One more related issue to be considered is the finite size of the gas jet and of the ion beam. For the current setup at the ESR, this leads to an extended interaction volume comparable with a cube of about $5 \mathrm{~mm}$ side length. This, in turn, results in a variation of the photon observation angle which causes a broadening of the observed x-ray lines. In order to reduce this effect, a novel target with a smaller diameter and/or employing an ion beam energy as low as possible with a small energy spread is desirable. CRYRING will offer these conditions. In addition, the high granularity of the microcalorimetric detectors with small pixels (covering small solid angles) will provide an intrinsic high resolution. Therefore, the Lamb shift experiments at CRYRING with small ion-atom/molecule interaction volumes in combination with high-resolution X-ray calorimeters will provide optimal conditions for much improved accuracy.

\section{Strong field QED in He-like heavy ions: electron-electron correlation in extreme fields}

The proposed experimental scheme at the electron cooler of the CRYRING (see Figure 2.3) can also be exploited for precision measurements of differences between ground-state binding energies of hydrogenand helium-like heavy ions (for the same nucleus). These differences can be obtained by accurately measuring energies of $\mathrm{x}$-rays emitted during the K-shell RR into initially bare and H-like ions. This technique, first introduced at the Lawrence Livermore National Laboratory Super-electron-beam iontrap (EBIT) facility (Marrs et al., 1995) has also already been successfully applied at the electron cooler of the ESR (Gumberidze et al., 2004). The proposed experimental scheme with high-resolution microcalorimeters, will enable us to significantly improve the precision of such measurements as compared to the previous attempts. This allows for stringent tests of high-order QED effects on the electron-electron interaction in the presence of extremely strong electromagnetic fields generated by the heavy nucleus. In these strong external fields, the influence of relativistic and QED effects on the bound electrons is significantly enhanced. As a consequence, the correlation (many-body) correction and the specific QED corrections for the electron-electron interaction are of the same order of magnitude. This provides a challenge for an accurate theoretical description. In addition, it is important to note that the influence of the uncertainty introduced by the poorly known extended size of the nucleus is negligible for the theoretical value of the two-electron contribution to the ground-level binding energy in He-like systems Marrs et al. (1995). These distinctive features make this particular kind of studies unique among other QED addressing, e.g., the $1 s$ Lamb shift (in one-electron systems), $g$-factors of bound electrons, or hyperfine splittings in high- $Z$ ions.

In summary, the experimental scheme proposed for the CRYRING is precisely tailored for an accurate determination of the $1 s$ Lamb shift in a very high- $Z$ hydrogen-like ion. If the conditions discussed are realized, an energy uncertainty of even significantly below $1 \mathrm{eV}$ can be anticipated. This will provide a real challenge for our current understanding of the atomic structure within the framework of QED.

\subsubsection{Towards supercritical fields}

The study of transient superheavy quasi-molecules with $Z_{\mathrm{UA}} \geq 173$, where $Z_{\mathrm{UA}}=Z_{\text {proj }}+Z_{\text {target }}$, in adiabatic collisions of very heavy projectiles like $\mathrm{Pb}$ and $\mathrm{U}$ with heavy targets has been a key field of 
investigation at GSI since its beginning (Greiner et al., 1985). In very heavy quasi-molecular collision systems the $1 s \sigma$ molecular orbital (MO) and the evolution of its energy have been in the focus of experimental and theoretical endeavours since the prediction of its diving into the Dirac sea for transient supercritical systems. The predicted very strong dependence of MO-energies on various relativistic and QED effects already for $Z \geq 137$ (effects, whose individual $Z$-dependencies follow $Z^{n}$ with mostly $n \geq 4$ ) have given rise to new questions about the character of the actual diving of molecular orbitals into the Dirac sea.

CRYRING in combination with the existing ESR storage ring will open unprecedented possibilities for investigation of the $1 s \sigma$ molecular orbital in these transient superheavy quasi-molecules. Storage ring technology has now reached the point where intense beams of bare $\mathrm{U}^{92+}$ and $\mathrm{H}$-like $\mathrm{U}^{91+}$ can be produced and stored at energies of well below $10 \mathrm{MeV} / \mathrm{u}$. This possibility to provide H-like and bare projectiles at low collision velocities is the all-decisive step beyond the experimental situation that existed previously. The experiments in the early days of GSI depended on the production of a vacancy in the $\mathrm{K}$ shell of one of the collision partners on the incoming part of the trajectory in order to be able to observe positrons or an $\mathrm{MO}$ x-ray emitted near the turning point of the trajectory. In contrast, storage rings permit experiments to be conducted where every projectile brings a K-shell vacancy into the collision. This facilitates a signal enhancement of up to seven orders of magnitude.

With these new conditions high resolution experiments on electron-, positron- and MO x-ray emission come into reach now. In particular, those phases of the collisions can be studied in detail where for bare and $\mathrm{H}$-like projectiles in the course of a sufficiently close encounter near the turning point $R_{0}$ an empty or singly occupied $1 s \sigma$ MO may be diving into the Dirac sea (see also Figure 2.4). It is emphasized that the CRYRING in combination with ESR is an optimum facility for these experiments with ESR providing the highly ionized ions and CRYRING facilitating their storage at comparatively low energies. For a sensible investigation of quasi-molecules one has to take into account that their 'lifetime' during a collision quickly decreases with increasing collision velocity. This makes CRYRING such a supremely attractive facility for all studies of transient superheavy quasi-molecules.

Experiments in CRYRING will begin with symmetric systems where $\mathrm{Xe}^{54+}-\mathrm{Xe}$ is most favorable, as this system can be directly investigated with existing supersonic gas jets and existing in-ring electron and photon spectrometers and reaction microscopes. All experiments will study collision dynamics and molecular orbital structure of transient superheavy quasi-molecules via the emission of electrons, positrons or $\mathrm{x}$ rays in coincidence with outgoing recoil ions. The measurement of the projectile scattering angle will provide the impact parameter, and hence, the internuclear distance during the collision event (Tupitsyn et al., 2010, 2012). For experiments with superheavy systems with $Z_{\mathrm{UA}}>137$ the system $\mathrm{Hg}^{80+}-\mathrm{Hg}$ is an attractive first candidate: the promising 2D-3D magnetooptical traps (MOTs) for $\mathrm{Hg}$ described in the literature shall be adapted to operation in the storage ring in order to offer the possibility for a symmetric truly superheavy collision system. A homologue 2D-3D MOT for Li could successfully be implemented into the TSR at the Max-Planck-Institut für Kernphysik (MPIK), Heidelberg by D. Fischer (see Section 2.2.5).

The previously developed technique (Tupitsyn et al., 2010) for evaluation of charge-transfer and electron-excitation processes in low-energy heavy-ion collisions has been extended by Tupitsyn et al. (2012) to collisions of ions with neutral atoms. With this method the $\mathrm{K}-\mathrm{K}$ charge transfer and $\mathrm{K}$ vacancy production probabilities for the $\mathrm{Ne}-\mathrm{F}^{8+}(1 s)$ collisions at $\mathrm{F}^{8+}(1 s)$ projectile energies 130 and $230 \mathrm{keV} / \mathrm{u}$ were evaluated. The obtained results were found to be in a good agreement with experimental 


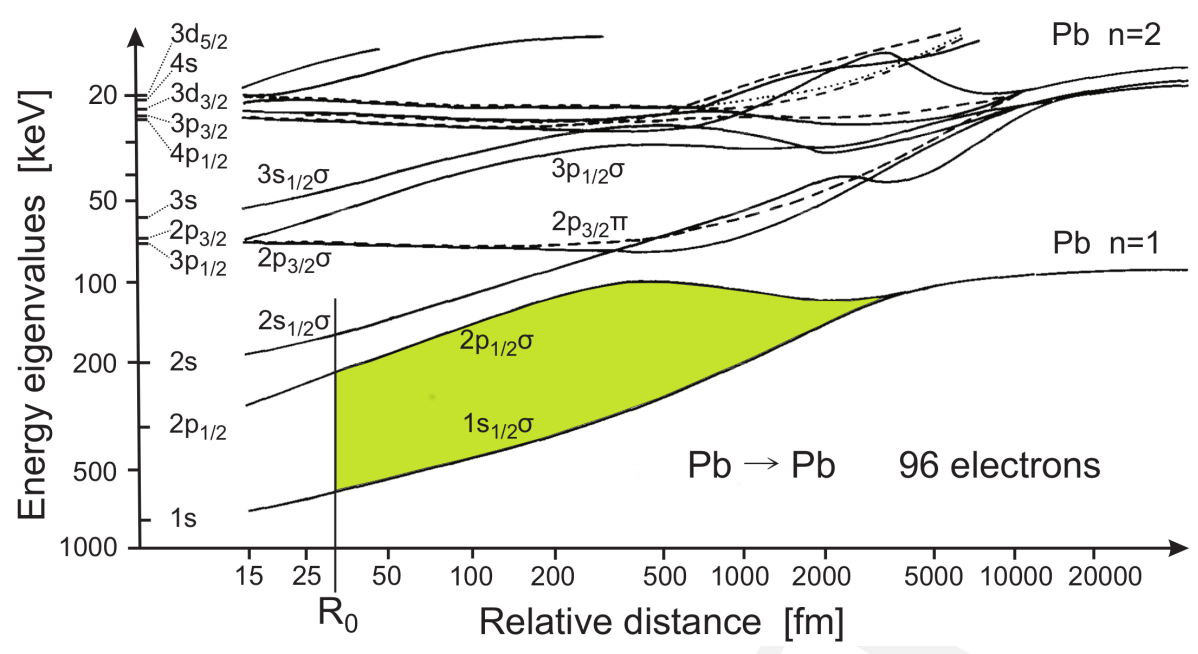

Figure 2.4. Correlation diagram for the symmetric $\mathrm{Pb}-\mathrm{Pb}$ collision system highlighting the area over the energy difference of the two innermost molecular orbitals (MO) $1 s \sigma$ and $2 p \sigma$ along a trajectory whose distance of closest approach is $R_{0}$. Calculations of the relativistic correlation diagram are from Sepp et al. (1981). The $\mathrm{Pb}-\mathrm{Pb}$ system was chosen here for illustration of the effects to be expected. It prominently features the very strong relativistic effects for the innermost MOs, which materialize as a continuous dramatic increase in the binding energy with decreasing internuclear separation $R$ down to very small internuclear distances corresponding to the united-atom limit. This is partly due to the very strong relativistic shrinking of the wave function of the innermost orbitals and is in strong contrast to the levelling off seen for the outer orbits. It is apparent from the logarithmic scale of the energy that — unique for very heavy collision systems like $\mathrm{Pb}-\mathrm{Pb}$ - even small decreases in the impact parameter below $200 \mathrm{fm}$ will contribute considerably to the accumulation of phase, thus providing enhanced sensitivity and accuracy for the derivation of energies of molecular orbitals during the encounter.

data (Abufager et al., 2004; Hagmann et al., 2010). The corresponding calculations were also performed for the $\mathrm{Xe}-\mathrm{Xe}^{53+}(1 s)$ collision system and, in principle, can be extended to higher- $Z$ systems, including, for instance, the $\mathrm{U}-\mathrm{U}^{91+}(1 s)$ collision system. However, the calculations for higher- $Z$ systems and especially in case of the supercritical regime, when the ground-state of the united quasimolecule can dive into the negative-energy Dirac continuum, require a proper treatment of the negative-energy continuum in the framework of the second quantization formalism. Such theoretical work is currently under way and would require benchmarking through precision experimental data. Such data are unavailable now and may become accessible for the first time in experiments at CRYRING@ESR. We expect also that the methods developed can be adopted for calculations of the ionization cross sections in low-energy ion-atom collisions.

\subsubsection{Atomic structure: electron-ion collisions}

Collisions of highly charged ions with free electrons offer numerous possibilities for fundamental studies of the atomic electron shell and of manifestations of nuclear effects on the atomic properties. Experiments on ionization, recombination, and excitation of ions, as well as on resonant scattering provide sensitive observables for stringent tests of modern atomic theory with respect to QED, many-body, and relativistic effects.

They also provide deep insight into processes relevant in astro- and plasma physics as well as in accelerator design (Müller, 2008). Moreover, nuclear properties can be quantitatively accessed by their effects on the electronic shell such as shifts of resonance energies due to nuclear-volume effects (Brandau et al., 2008), level splitting due to the nuclear magnetic moment (Lestinsky et al., 2008), and by their 
impact on the lifetimes of metastable levels via hyperfine quenching (Schippers et al., 2005, 2007, 2012). A direct coupling of the incident electron to the nucleus mediated by resonant nuclear excitation, the nuclear excitation by electron capture (NEEC) process (see Section 3.2), has been predicted theoretically. Investigations employing electron-ion collision spectroscopy are not restricted to ions with stable nuclei but can be extended to radioactive species provided their lifetime is sufficiently long (about $10 \mathrm{~s}$ or longer) for beam cooling and measurements (Brandau et al., 2009, 2010, 2013).

Beams of electrons with small energy and momentum spreads can be readily produced over a very wide range of energies. They provide access to regimes where optical methods are not available, in particular, for directly probing the innermost energy levels in the ionic electron shell of heavy nuclei. Furthermore, electron beams are, compared to foils or gas-jets, dilute targets that ensure single collision conditions, and the electrons themselves are fundamental particles without a known internal structure. Collisions between electrons and stored ions can be realized in two different geometric arrangements, using either merged or crossed beams. The existing electron cooler at CRYRING naturally provides a merged-beams configuration. It was already successfully used in electron-ion collision experiments in the past. A particular strength of the merged-beams arrangement is due to its special kinematics which gives highest energy resolution at small collision energies.

An alternative approach to in-ring electron-ion collisions is an intense crossed-beams electron target which will be discussed in more detail below (Section 5.4). In a transverse electron-target arrangement, the low collision energy domain is not accessible for kinematic reasons. However, much higher electron densities (up to $10^{9} \mathrm{~cm}^{-3}$ ) compared to those available at the electron cooler (typically $10^{7} \mathrm{~cm}^{-3}$ ) can be provided for systematic measurements at high collision energies. The electron-ion interaction region is much more accessible in a crossed-beams arrangement than in an electron cooler which is surrounded by heavy solenoids providing the magnetic guiding field for the cooler electron beam. Therefore, as a further advantage, a dedicated transverse electron target can accommodate comparatively large solid angles for the observation of photons from the electron-ion collision region.

The electron cooler of CRYRING was the first to employ adiabatic magnetic expansion of the electron beam (Danared, 1993). This technique reduces the transverse velocity spread of the electrons inversely proportional to the magnetic expansion factor. Experiments in Stockholm demonstrated transverse and longitudinal electron temperatures of $k_{B} T_{\perp}<1.5 \mathrm{meV}$ and $k_{B} T_{\|} \approx 0.05 \mathrm{meV}$, respectively. With a maximum magnetic expansion ratio of 100 , the transverse electron temperature of the CRYRING cooler is up to two orders of magnitude lower than that of the ESR cooler. It should be noted that in addition to the strongly suppressed transverse electron velocity spread longitudinal temperatures in the CRYRING cooler appear to be a factor of 2-4 lower than those of the ESR cooler. This difference can be explained by suppression of relaxation processes between the transverse and longitudinal degrees of freedom.

Even though negative electron affinity GaAs photocathodes nowadays set a benchmark for low electron-beam temperature (Orlov et al., 2005), the CRYRING electron cooler is a competitive device, in particular, if one keeps in mind that the operation of a GaAs photocathode is much more involved as compared to the operation of a conventional thermionic cathode. Moreover, thermionic cathodes still provide much higher electron current densities than state-of-the-art photocathodes, which strongly helps experiments with low signal-to-noise ratios.

A simulation of achievable resonance widths with the electron temperatures of the ESR electron cooler and of the CRYRING electron cooler illustrates the vastly improved resolving power accessible 

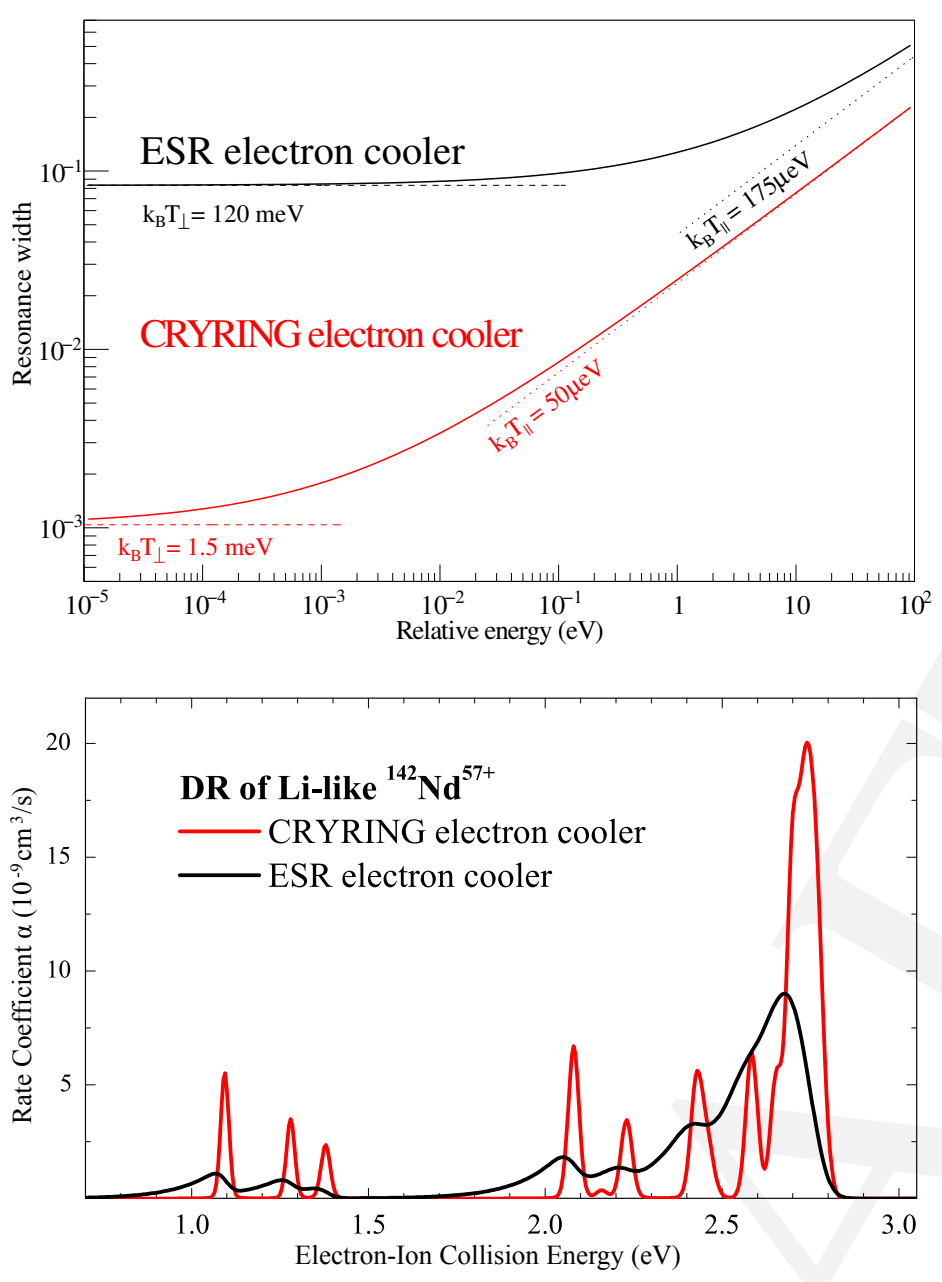

Figure 2.5. Comparison of the obtainable energy resolutions in merged-beams experiments with the electron coolers in CRYRING (red curve) and ESR (black curve). The contributions to the energy spread by the longitudinal electron temperature $k_{B} T_{\|}$ and the transverse temperature $k_{B} T_{\perp}$ are indicated with dashed and dotted lines, respectively. The CRYRING electron cooler offers a resolution gain of almost two orders of magnitude at small relative energies and of still a factor $\gtrsim 2$ improvement at relative energies above $100 \mathrm{eV}$.

Figure 2.6. A calculated DR spectrum of Li-like ${ }^{142} \mathrm{Nd}^{57+}$ (compare Figure 3.1) under the experimental conditions at the electron cooler of the ESR (red line) and at the CRYRING electron cooler with an adiabatically expanded beam. The assumed temperatures of the electron beams are $k_{B} T_{\perp}=120 \mathrm{meV}$ and $k_{B} T_{\|}=175 \mu \mathrm{eV}$ for the ESR cooler and $k_{B} T_{\perp}=1.5 \mathrm{meV}$ and $k_{B} T_{\|}=50 \mu \mathrm{eV}$ for the CRYRING electron cooler.

at the CRYRING cooler (see Figure 2.5). The resolution gain at the CRYRING cooler is particularly pronounced at small electron-ion collision energies. Due to conservation of resonance strength both the signal-to-background-ratio and the total count rate "on resonance" improve with larger resolving power yielding overall significantly enhanced sensitivity. Example experiments benefiting from the improved experimental conditions will be laid out and discussed next.

\section{QED tests employing high-resolution collision spectroscopy of heavy few-electron ions}

The combination of beams of ions up to the highest possible charge states and a cold electron target with a transverse temperature of roughly a factor 100 below that of the ESR cooler and still a factor of 2-4 lower longitudinal temperature will allow for high-resolution dielectronic recombination (DR) measurements and according high-precision collision spectroscopy. One of the decisive benefits of an improved energy resolution is the possibility to more accurately determine resonance positions (compare Figure 2.6). Moreover, as a consequence of the low transverse temperature, according asymmetric line shapes and shifts in energy are almost completely removed. Uncertainties caused by the transverse temperature were one of the largest sources of experimental errors in recent DR spectroscopic studies at the ESR (Bernhardt et al., 2015a,b).

The increased accuracy, resolution and sensitivity of DR collision spectroscopy facilitates Lambshift measurements with heavy highly charged ions, e.g., of $2 s-2 p_{j}$ transitions in Li-like heavy ions with unprecedented precision Brandau et al. (2015). Two alternative approaches are currently pursued. 
The first technique utilizes the fact, that in selected cases, the excitation energy of a core electron and the binding energy of the $n \ell_{j}$ electron (which should preferentially be captured into a shell with a high principal quantum number $n$ ) almost completely cancel. The second technique is based on the regular pattern of Rydberg series of resonances.

When the excitation energy of an initially bound electron almost matches the binding energy of the initially free electron resonances may occur near zero electron-ion collision energy. In this case an exceptional precision can be achieved as demonstrated in various experiments at CRYRING and TSR (Lindroth et al., 2001; Kieslich et al., 2004; Schuch et al., 2005; Lestinsky et al., 2008). We note that the operation of TSR was stopped at the Max-Planck Institute for Nuclear Physics in Heidelberg. The second, more general method is well-suited for the determination of excitation energies of very heavy ions. It exploits the almost hydrogenlike high- $n$ behaviour of Rydberg series associated with a given atomic excitation channel. This method has been developed and established at the ESR electron cooler for $2 s-2 p_{1 / 2}$ transitions in Li-like gold, lead and uranium (Spies et al., 1995; Brandau et al., 2003) and was used recently for determining transition energies of Li-like and Be-like xenon ions (Bernhardt et al., 2015a,b). At CRYRING, for both approaches an improvement in accuracy of at least one order of magnitude compared to the present ESR conditions can be expected, i.e., an improvement to the level of below $10 \mathrm{meV}$. These favorable experimental conditions will facilitate sensitive tests of higher-order QED contributions to atomic transition energies. Electron-ion collision spectroscopy is thus definitely competitive with the most advanced x-ray spectroscopy methods Beiersdorfer et al. (2005). The high resolution that can be expected at CRYRING also opens possibilities to study predicted changes in line shapes of DR resonances due to interference with radiative recombination (RR) or with other DR resonances of identical symmetries (Schippers et al., 1998; Andreev et al., 2008). This is particularly promising for applications to highly charged ions that can be studied at the combined CRYRING@ESR facility.

\section{Spin-flip transitions in the relativistic domain}

In DR of fluorine-like ions the lowest-energy core excitation is between fine-structure levels of the $2 p^{5}$ configuration. Such transitions are forbidden in the LS-coupling scheme and have therefore been neglected in previous theoretical treatments. Experiments with F-like iron ions have, however, shown sizable contributions of $e+2 p^{5}{ }^{2} P_{3 / 2} \rightarrow 2 p^{5}{ }^{2} P_{1 / 2} n l$ processes involving a core spin-flip transition (Savin et al., 1997). With very highly charged ions such as $\mathrm{U}^{83+}$ these processes can be studied in the relativistic domain. The fine-structure splitting within the $2 p^{5}$ configuration of high- $Z$ ions is in the keV-range and thus well suited for high-resolution measurements at the CRYRING cooler.

\section{Hyperfine DR}

While excitations within fine-structure levels of a given electronic configuration feature the lowest possible energies for ions with even-mass nuclei, still much lower excitation energies are possible when the nucleus has non-zero spin and, hence, a non-vanishing magnetic moment. Similar to the finestructure transitions the core excitation involves a spin flip. Theoretical treatment requires the inclusion of relativistic effects. The first calculations of this kind for DR involving hyperfine transitions were carried out by Pindzola et al. (1992) for DR processes $\mathrm{Bi}^{82+}(1 s F=4)+e^{-} \rightarrow \mathrm{Bi}^{81+}(1 s F=5 n l)$. DR resonance energies $E_{n}$ can be estimated from the Bohr formula for the binding energy of the Rydberg electron, i.e., $E_{n}=E_{\text {exci }}-13.6 \mathrm{eV} \times q^{2} / n^{2}$ with the initial ion charge state $q$. The inner-shell 
$(1 s F=4) \rightarrow(1 s F=5)$ hyperfine splitting is only $E_{\text {exci }}=5.08 \mathrm{eV}$ (Klaft et al., 1994). With this value for $E_{\text {exci }}$ and with $q=82$, the lowest Rydberg quantum number which yields a positive resonance energy $E_{n}$ is $n_{\min }=135$. Such highly excited fragile Rydberg states are easily field-ionized in the motional electrical field $\vec{E}=\vec{v} \times \vec{B}$ that the recombined ions are subjected to in the storage ring dipole magnets (Schippers et al., 2001). A classical formula for the lowest quantum number $n_{\text {cut }}$ that is affected by field ionization is $n_{\text {cut }}(F)=\left(5.71 \times 10^{8} \mathrm{~V} / \mathrm{cm} \times q^{3} / F\right)^{1 / 4}$.

Typical ESR experimental conditions are such that $n_{\text {cut }}<n_{\min }$, i.e., the observation of hyperfine DR is prevented by field ionization. However, the condition $n_{\text {cut }}>n_{\min }$ can easily be fulfilled in CRYRING which can store highly charged ions at much lower ion velocities $v$ and correspondingly lower fields $\vec{E}=$ $\vec{v} \times \vec{B}$ as compared to ESR. Electron-ion collision spectroscopy of low-energy hyperfine DR resonances holds the promise to yield experimental values for $(1 s F=4) \rightarrow(1 s F=5)$ hyperfine transition energy on the 5 ppm level (Lestinsky et al., 2008) which would even exceed the performance of laser spectroscopy of such transitions (Nörtershäuser, 2011; Ullmann et al., 2015).

A prerequisite for the accurate determination of hyperfine DR resonance energies is the high experimental resolving power of the CRYRING electron cooler. But even with the energy resolution that is possible at the CRYRING cooler, experiments will be difficult because of the high background cross section of RR However, it is merely a matter of getting sufficiently good statistics to see isolated DR resonances associated with hyperfine transitions. Conditions for lower- $Z$ ions may even be better than for $\mathrm{Bi}^{82+}$ because the lower $Z$ will reduce the $\mathrm{RR}$ cross section and at the same time the Rydberg resonances to be observed become narrower (and thus more distinct) due to their reduced radiative widths. Hydrogenlike ions with atomic numbers down to about $Z=60$ feature ground-state hyperfine splittings of more than $1 \mathrm{eV}$. Even ${ }^{113} \mathrm{In}^{48+}$ still has a predicted hyperfine splitting of $0.91 \mathrm{eV}$. Thus, a wide range of nuclei, including radioactive species, are accessible by hyperfine DR experiments which can be used to measure the splitting with high accuracy. The CRYRING cooler with its low transverse electron temperature is ideal for such studies.

\section{Ionization via resonant and non-resonant inner-shell excitation}

Electron-impact ionization of atoms and ions can proceed via three different channels:

- direct removal of an (outer-shell) electron,

- excitation of an inner-shell electron with subsequent autoionization,

- resonant excitation, i.e., capture of the incident electron with simultaneous inner-shell excitation followed by the emission of two electrons.

The latter process is in competition with DR where the intermediate multiply excited state populated by dielectronic (resonant) capture decays by the emission of radiation. When the atomic number increases, the stabilizing-transition rates can change dramatically which is especially true for radiative transitions which are forbidden in the LS coupling scheme with low- $Z$ atoms or ions. With dependences of transition rates from such states proportional to $Z^{10}$ it becomes clear, that high- $Z$ dielectronic capture with subsequent stabilization by the emission of electrons or photons can dramatically change the cross sections for recombination or ionization or resonant elastic scattering. Chen and Reed (1993) have investigated the influence of relativity and of including 'forbidden' transitions on the resonant contributions to electron-impact ionization of highly charged lithium-like ions and found dramatic effects both in the resonance energies and in the resonance strengths. Such strong effects can already be seen in Li-like $\mathrm{Fe}^{23+}$ where resonances in the ionization cross section occur at energies above $6.8 \mathrm{keV}$. With ESR as an 
injector CRYRING can accommodate even more highly charged ions for such studies. In the electron-ion merged-beams arrangement resonances and excitation-autoionization steps can be observed with good energy resolution providing unique access to possible shifts of energies due to relativistic effects.

Since the electron-ion center-of-mass energy range accessible with the cooler at CRYRING will be limited to only several $\mathrm{keV}$, resonances in electron-impact ionization will be accessible only for relatively low- $Z$ Li-like ions. However, for Na-like ions the energy is sufficient up to about $\mathrm{Xe}^{43+}$ where strong resonances and excitation-autoionization features are predicted (Reed et al., 1990) which are expected to dominate the commonly known direct-ionization channel. Higher collision energies will be available with an in-ring transversal electron target (Sec. 5.4) which will, thus, provide access to electron-impact excitation and ionization of even more highly charged ions.

\subsubsection{Atomic lifetimes}

Radiative transition rates in atomic systems are among the fundamental entities needed for any description of atomic structure and dynamics, for the modeling of any plasma, and for synthetic spectra based on collisional-radiative modeling as a means to interpret and make use of observations of terrestrial and astrophysical plasmas (Träbert, 2014). Accurate measurements of transition rates of, in particular, exotic decay modes such as hyperfine induced (HFI) or two-photon transitions still pose a challenge for state-of-the-art atomic structure theory (Johnson, 2011; Fritzsche et al., 2015).

The most common transition type, electric dipole (E1) transitions, has been observed and studied in many light sources. As far as highly charged ions (HCIs) are concerned, beam-foil spectroscopy is the presently only practical technique to study E1 transition rates. This technique is suitable for level lifetimes in the range from many nanoseconds to a few picoseconds, and it has been employed at GSI in a number of experiments (Möller et al., 1989; Träbert et al., 1994, 1995; Toleikis et al., 2004; Zou et al., 2005). There are excited levels that for atomic symmetry reasons cannot decay by E1 transitions. For example, the lowest excited level in He-like ions, $1 s 2{ }^{3} S_{1}$, decays by a relativistic magnetic dipole (M1) transition; the lifetime of this level in $\mathrm{Ar}^{16+}(200 \mathrm{~ns})$ has been measured employing a beam of recoil ions in the GSI Stripper Hall (Hubricht and Träbert, 1987).

Ion-storage ring provides an ideal environment for measuring even longer atomic lifetimes because of the long ion-storage times and, hence, observation times that can be realized. At CRYRING in Stockholm, research specialized on singly charged ions from a special ion source, employing laser-probing of the level population as a function of time after ion injection into the storage ring and determining mostly very long level lifetimes up to the one-minute range (Mannervik, 2003). At TSR in Heidelberg, lifetimes ranging from one millisecond to many seconds were measured on spin-forbidden E1, E1-forbidden M1 and E2, or HFI transitions by optical detection of fluorescence photons (Träbert, 2000, 2002). In addition, long-lived atomic states associated with rare decay modes, such as M1 (Schmidt et al., 1994; Saghiri et al., 1999) or HFI (Schippers et al., 2007, 2012) were investigated by means of DR spectroscopy (Section 3.1). For some decay types and the millisecond lifetime range, EBITs (especially those at Livermore and Heidelberg) are a viable competition that, in some cases, leads the field with respect to accuracy (e.g., Beiersdorfer et al., 2016). The excitation conditions in all the ion sources are different, and this diversity is necessary for systematic cross checks on the various techniques.

At the ESR, the transition between the hyperfine levels of the ground state of H-like bismuth and uranium ions (Klaft et al., 1994; Seelig et al., 1998; Ullmann et al., 2015) has been induced by laser 
light (massively shifted in the rest frame of the fast ions), and an upper level lifetime in the millisecond range was measured (Klaft et al., 1994). Furthermore, DR spectroscopy was used to explore two-photon transitions in Be-like xenon ions (Bernhardt et al., 2012, 2015b). Hence, GSI has already demonstrated mastering atomic lifetime measurements on very highly charged ions. CRYRING@ESR will open access to a multitude of experiments on longer-lived atomic levels and thus on the specific internal symmetries of various atomic systems. The excellent vacuum conditions of CRYRING allow for long storage times at low kinetic energies such that quenching of the metastable levels through external fields can be largely avoided.

There are several parameter ranges with quite a number of valuable research opportunities which can be addressed in CRYRING@ESR.

1. When CRYRING was operated at the Manne-Siegbahn Laboratory in Stockholm, the ion species $\mathrm{A}^{q+}$ studied were limited to $q=1$ by the chosen source. There are various low charge state ions of heavy elements of astrophysical or fundamental interest. Because of this interest, some species have been studied in conventional ion traps decades ago, but with only moderate accuracy (few to some ten percent) — this might be improved in CRYRING@ESR to the one-percent range which is much more challenging to theory.

2. The injector at TSR in Heidelberg was limited in ion beam energy and in ion species compared to the GSI accelerator facilities. ESR as injector for CRYRING can provide highly charged ions of all elements. CRYRING@ESR will thus open up an entirely new range of lifetime measurements with HCI.

3. Theory on hyperfine-induced decays has been improved recently (Cheng et al., 2008; Andersson et al., 2009; Li and Dong, 2010), improving the agreement with experiment to some extent, but not conclusively. For the ion species of interest, a broader experimental basis is desirable. New measurements will require $\mathrm{x}$-ray detection with sufficiently good energy resolution. The required resolution can be provided, for example, by microcalorimeters.

4. There are moderately high charge state ions in which M2 and E2 decays become important (with increasing nuclear charge). While extreme ultraviolet (EUV) observations are difficult because of the necessary combination of detection efficiency and spectral resolution, this should not be a problem for various $\mathrm{x}$-ray transitions.

5. A particular class of exotic transitions, the two-photon E1M1 decay of Be-like ions in the metastable $2 s 2 p^{3} \mathrm{P}_{0}$ level has attracted considerable attention recently (Bernhardt et al., 2012, 2015b; Fritzsche et al., 2015). The lifetimes are unexpectedly long due to relativistic effects. For the heaviest Belike ions they come into reach of storage-ring measurements. Experiments with species such as $\mathrm{U}^{88+}$ are planned for CRYRING@ESR.

Most of the ions of interest can be provided by the GSI backbone accelerators. A rule of thumb from the TSR passive observation experiments calls for stored ion currents of $I>1 \mu \mathrm{A}$ particles to reach a satisfying signal-to-noise ratio. The passive photon observations or DR spectroscopy do not require any specific ion beam energies, so that it may be convenient to use parasitic beams from the backbone. Some of the suggested measurements can be done without the big machines (for example, during commissioning phases) using CRYRING's stand-alone injector. 


\subsubsection{Polarization spectroscopy of hyperfine transitions in helium-like ions: A novel approach to study nuclear magnetic moments}

Significant progress has been achieved recently in applying atomic spectroscopy methods to investigate the nuclear structure of radioactive as well as stable isotopes. For instance, a number of laser-supported collision experiments are currently prepared at the ESR storage ring and developed for the FAIR facility. In these studies, optical techniques, based on hyperfine structure splitting shall provide precise information about nuclear spins, moments and charge radii of various isotopes. In contrast to these measurements, which mainly deal with the transition energies and total rates, much less attention has been paid until now to polarization-resolved analysis of hyperfine transitions not only in the optical but also in the x-ray regime. Owing to the recent advances in X-ray detector techniques (Märtin et al., 2012) such an analysis may provide an alternative route for probing properties of stable nuclei as well as of exotic isotopes far from stability.

Figure 2.7. Degree of linear polarization of the $F_{i}=$ $3 / 2 \rightarrow F_{f}=1 / 2$ hyperfine line (left panel) and the $1 s 2 p_{3 / 2}{ }^{3} P_{2} \rightarrow 1 s^{2}{ }^{1} S_{0}$ transition (right panel) following radiative electron capture into the $1 s 2 p_{3 / 2}{ }^{3} P_{2}$ state of the finally helium-like thallium ${ }^{205} \mathrm{Tl}^{79+}$ ion with projectile energy $T_{p}=100 \mathrm{MeV} / \mathrm{u}$. Calculations are performed within the magnetic-quadrupole approximation (dashed line) and by taking the hyperfine induced $E 1-$ M2 mixing into account.
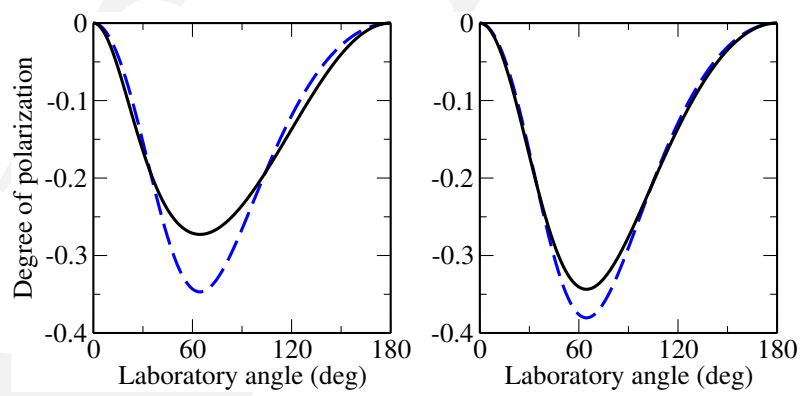

In order to illustrate the potential application of the x-ray polarimetry to the analysis of the nuclear properties, theoretical investigations of the linear polarization of $1 s 2 p_{3 / 2}{ }^{3} P_{2} \rightarrow 1 s^{2}{ }^{1} S_{0}$ decay of heliumlike ions with non-zero spin have been recently performed (Surzhykov et al., 2013; Wu et al., 2014, 2015). Because of the hyperfine interaction, which mixes the excited $2{ }^{3} P_{2}$ state of such ions with the short-lived $2^{1,3} P_{1}$ levels, the $2{ }^{3} P_{2} \rightarrow 1{ }^{1} S_{0}$ decay can proceed via the quenched electric-dipole $E 1$ channel in addition to the leading $M 2$ transition. Owing to the angular momentum selection rules not all individual hyperfine lines, that give rise to the overall $2{ }^{3} P_{2} \rightarrow 1{ }^{1} S_{0}$, are influenced by the $E 1-M 2$ mixing. In the ions with the half-integer nuclear spin, $I=1 / 2$, for example, the hyperfine-quenched $M 2$ channel is allowed only for the $F_{i}=3 / 2 \rightarrow F_{f}=1 / 2$ transition. The linear polarization of this transition:

$$
P_{i f}(\theta)=\frac{\sum_{k=2,4, \ldots} \sqrt{\frac{16 \pi}{2 k+1}} \mathscr{A}_{k}\left(\alpha_{i} F_{i}\right) g_{k} Y_{k 2}(\theta, 0)}{1+\sum_{k=2,4, \ldots} \mathscr{A}_{k}\left(\alpha_{i} F_{i}\right) f_{k} P_{k}(\cos \theta)},
$$

is defined - apart from the alignment $\mathscr{A}_{k}$ of the initial ionic state—by the structure functions:

$$
f_{2} \approx 1-2 \sqrt{3} \frac{a_{E 1}}{a_{M 2}}, g_{2} \approx 1+\frac{2}{\sqrt{3}} \frac{a_{E 1}}{a_{M 2}} .
$$

Here we used the short-hand notation $a_{E 1, M 2}=\left\langle 1{ }^{1} S_{0} F_{i}=1 / 2\left\|H_{\gamma}(E 1, M 2)\right\| 2{ }^{3} P_{2} F_{f}=3 / 2\right\rangle$ for the reduced matrix elements of the hyperfine-quenched electric-dipole and the leading magnetic-quadrupole transitions. These amplitudes depend on the hyperfine-mixing coefficients and, consequently, on the nuclear magnetic dipole moment $\mu_{I}$. The analysis of the interference between the $E 1$ and $M 2$ transitions, as parameterized by Equation 2.2, may be used, therefore, as a tool for probing the nuclear dipole magnetic 
moments.

In the left panel of Figure 2.7 the linear polarization of the individual $F_{i}=3 / 2 \rightarrow F_{f}=1 / 2$ line is displayed following the radiative electron capture (REC) into the $1 s 2 p_{3 / 2}{ }^{3} P_{2}: F_{i}=3 / 2$ excited state of helium-like thallium ${ }^{205} \mathrm{Tl}^{79+}\left(I=1 / 2, \mu_{I}=1.638\right)$ ion. Calculations have been carried out for the projectile ion energy $T_{p}=100 \mathrm{MeV} / \mathrm{u}$ and for the different photon emission angles. Moreover, in order to illustrate the role of the E1-M2 mixing, predictions based on Equation 2.1-2.2 are compared with those obtained within the pure magnetic-quadrupole $M 2$ approximation, i.e. when the hyperfine interaction and, hence, the quenched $E 1$ transition are neglected. As seen from the figure, the admixture of the $E 1$ channel generally results in the reduction of the (degree of) linear polarization (2.1). The effect is most pronounced for an almost perpendicular photon emission, $\theta \approx 70^{\circ}$, where the absolute value of the polarization is maximal. For these angles, $P_{i f}$ decreases by about from $-35 \%$ to $-27 \%$ if apart from the leading magnetic-quadrupole $M 2$, the hyperfine-quenched $E 1$ decay channel is taken into account.

The experimental restrictions make it impossible to directly compare the calculated polarization of the individual $F_{i}=3 / 2 \rightarrow F_{f}=1 / 2$ transition with the measured data. Owing to a relatively small $(\sim 2 \mathrm{eV})$ energy separation between $F_{i}=3 / 2$ and $F_{i}=5 / 2$ states, their radiative decays following the REC can not be distinguished in the current investigations. In the right panel of Figure 2.7 we display, therefore, the linear polarization of the $2{ }^{3} P_{2} \rightarrow 1{ }^{1} S_{0}$ line, 'seen' as the incoherent superposition of its $F_{i}=3 / 2 \rightarrow F_{f}=1 / 2$ and $F_{i}=5 / 2 \rightarrow F_{f}=1 / 2$ components. Such an 'incoherent' summation is justified since the splitting between $F_{i}=3 / 2$ and $5 / 2$ levels is larger than their widths. Similar to before, calculations have been performed within the magnetic-quadrupole approximation and by taking a full account of the interference between the $E 1$ and $M 2$ transitions. Although in contrast to the $F_{i}=3 / 2$ state, the decay of the $F_{i}=5 / 2$ level can proceed only via the magnetic-quadrupole channel, the overall $2{ }^{3} P_{2} \rightarrow 1{ }^{1} S_{0}$ line is still remarkably affected by the $E 1-M 2$ multipole mixing.

The polarization analysis of the $E 1-M 2$ interference remains feasible even in the case when the fine-structure $1 s 2 p_{3 / 2}{ }^{3} P_{2} \rightarrow 1 s^{2}{ }^{1} S_{0}$ transition can not be resolved from the $1 s 2 p_{3 / 2}{ }^{1} P_{1} \rightarrow 1 s^{2}{ }^{1} S_{0}$ one. As we have recently shown, the linear polarization of the overall $K \alpha_{1}$ line is strongly affected by the hyperfine-quenched E1 channel and the effect becomes most pronounced for low-energy collisions (Wu et al., 2015). Based on these results, we argue that the linear polarization of the $\mathrm{K} \alpha_{1}$ line, which can be analyzed with available polarization detectors, may provide a novel tool to study the nuclear magnetic moments of stable as well as radio-active isotopes.

\subsubsection{Photoionization of stored ions}

Photoionization of ions occurs in many cosmic environments and man-made plasmas. An accurate knowledge of photoionization cross sections is mandatory for a detailed understanding of the charge balance in photoionized plasmas and, consequently, of the emitted radiation. In general, the atomic data needs for the modeling of nonequilibrium plasmas are vast. Most of the body of compiled data comes from theory which needs to apply approximations to make calculations tractable even with modern computer technology at hand. Therefore, benchmarking by experiment is vitally needed. The presently most advanced experimental technique for investigating photoionization of ions is the photon-ion merged beam technique (Schippers et al., 2015) where a beam of energetic photons from a synchrotron radiation source is collinearly merged with a beam of mass/charge selected ions from an ion source (Figure 2.8).

An inherent limitation of this method is that the internal state of ions from the ion source cannot be 


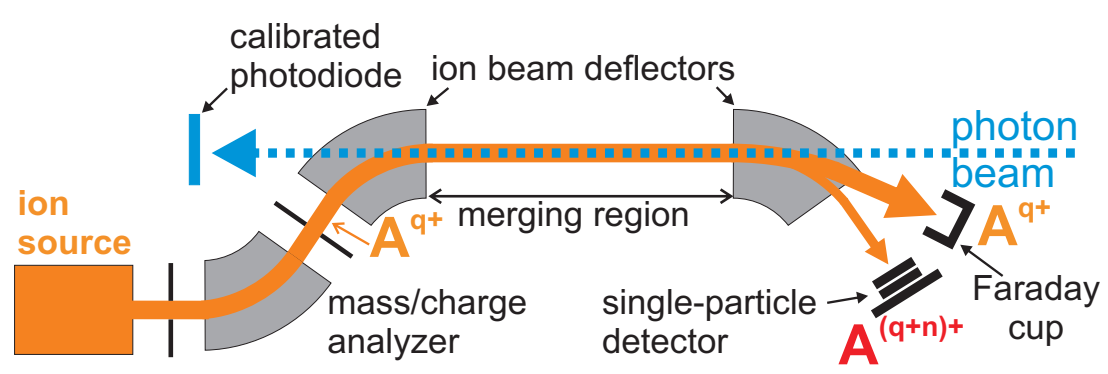

Figure 2.8. Essential features of a photon-ion merged-beams arrangement (Schippers et al., 2015). The reaction products $\mathrm{A}^{(q+n)+}$ result from $n$-fold ionization of the $\mathrm{A}^{q+}$ primary ions by photon impact.

controlled. In particular, long lived metastable levels survive the flight time of typically a few microseconds from the source to the photon-ion interaction region and thus contribute to the ionization signal in unknown quantity. An experimental environment that offers control of the internal degrees of freedom of ions is a heavy-ion storage ring (see, e.g., Schippers, 2015) where ions can be stored for sufficiently long times such that metastable levels can decay to the ionic ground state before data taking is started. The combination of an extreme ultraviolet (XUV) beam with a heavy-ion storage ring would thus be ideal for the measurement of absolute cross sections for the photoionization of atomic ions. In addition, the spectroscopy of photoionization resonances would provide a complementary experimental approach to unraveling the electronic structure of highly charged ions.

The setup of a storage ring at a synchrotron radiation facility would be a major investment and, in addition, would require much more floor space than usually available at a synchrotron light source. Therefore, all existing photon-ion merged-beams experiments at synchrotron light sources are based on singlepass arrangements (Kjeldsen et al., 1999a; Yamaoka et al., 2001; Covington et al., 2002; Gharaibeh et al., 2011; Schippers et al., 2014). We thus propose to install an intense source of XUV photons at a heavy ion storage ring.

Recent advances in laser technology have lead to the development of intense beams of XUV photons from comparatively small-scale lasers. Such laser systems are capable of providing photon fluxes exceeding $10^{12}$ Photon/s in the photon energy range 20-40 eV (Hädrich et al., 2014). Currently, research is focused on reducing the relative energy bandwidth of these sources to below $10^{-3}$ (Rothhardt et al., 2014, 2015). These conditions are almost comparable to what is available from undulator beam lines of 3rd generation synchrotrons. In addition, cooled ion beams in storage rings usually have higher particle densities than ion beams in single pass experiments. Thus, photoionization measurements using intense stored ion beams in combination with a state-of-the-art XUV laser are definitely feasible.

As a first step we will install an intense XUV laser at CRYRING and conduct proof-of-principle experiments using low-charged ions from the CRYRING injector. The experimental section YR09 (Section 5.1) of CRYRING will serve as the merging region. Photo ions can be directed onto a single-particle detector (e.g., Rinn et al., 1982) behind the first CRYRING dipole magnet following the interaction region. The arrangement would be very similar to the single-pass configuration sketched in Figure 2.8.

For the selection of a suitable ion species for a proof-of-principle experiment one has to consider several boundary conditions. First, the ionization energy should be within the accessible photon energy range $20-40 \mathrm{eV}$. Second, the mass-to-charge ratio of the ion and its kinetic energy must match the requirements of the injector and the storage-ring. A closer inspection suggests that $\mathrm{C}^{+}$might be a good candidate for a proof-of-principle experiment. $\mathrm{C}^{+}$ions were already stored in CRYRING, when it was 
still located in Stockholm, and used for electron-ion recombination experiments (Ali et al., 2012).

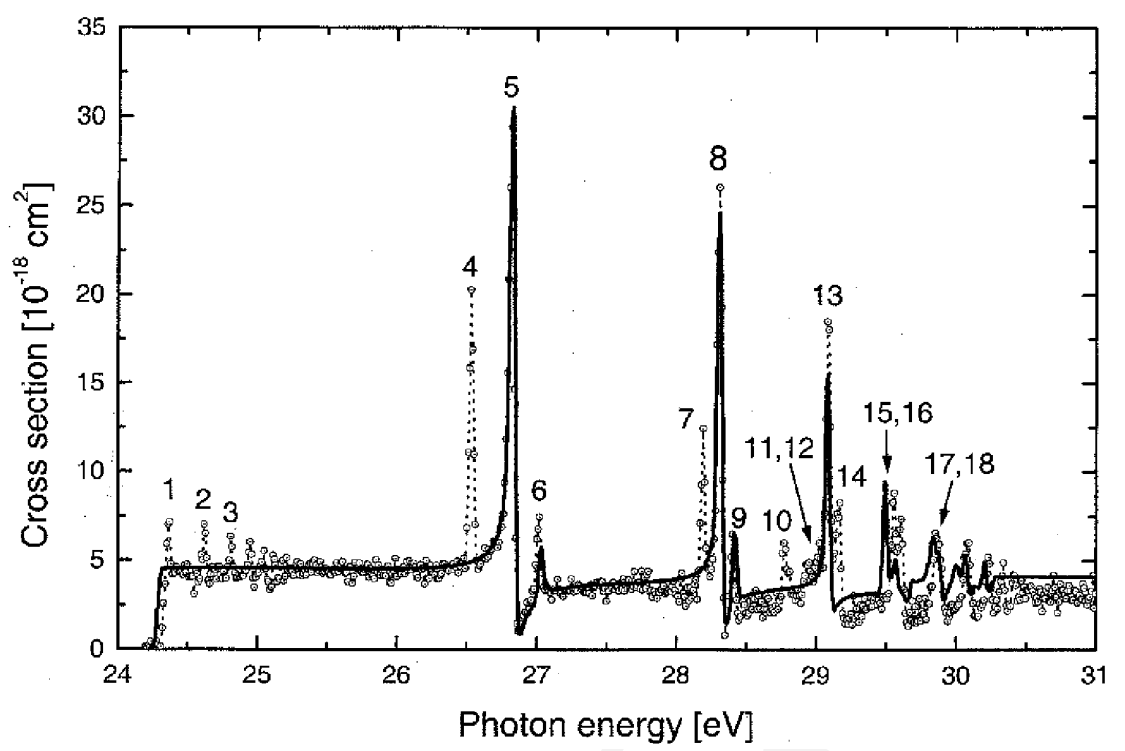

Figure 2.9. Measured (symbols) and calculated (full line) cross sections for photoionization of $\mathrm{C}^{+}$ions (Kjeldsen et al., 1999b). The resonances 4-18 are associated with the excitation of $2 s 2 p n p$ autoionizing resonances. The resonances $1-3$ are associated with the resonant ionization of initially metastable $\mathrm{C}^{+}\left(2 s 2 p^{24} P\right)$ ions.

Cross sections for photoionization of $\mathrm{C}^{+}$were measured at ASTRID storage ring in Århus, Denmark (Kjeldsen et al., 1999b, 2001). Figure 2.9 shows the corresponding results in the energy range around the threshold for the ionization of $\mathrm{C}^{+}$ions in the $2 s^{2} 2 p^{2} P$ ground term. The measured spectrum shows distinct signatures (resonances labelled by 1,2 , and 3) of initially metastable $\mathrm{C}^{+}\left(2 s 2 p^{2}{ }^{4} P\right)$ ions with lifetimes of up to about $100 \mathrm{~ms}$ (Träbert et al., 1999). In a storage ring experiment there would be sufficient time to allow for the decay of the metastable ${ }^{4} P$ levels, and a photoionization measurement could be performed with a pure ground-state beam.

The XUV radiation is created by high harmonic generation (HHG) of an infrared femtosecond fiber laser. In the spectral domain odd harmonics can be observed as shown in Figure 2.11. A single harmonic line can be isolated by a monochromator, thin metal foils or dielectric multi-layer mirrors. The relative energy bandwidth of a single harmonic is typically of the order of $10^{-2}$, depending on the generation

(a)

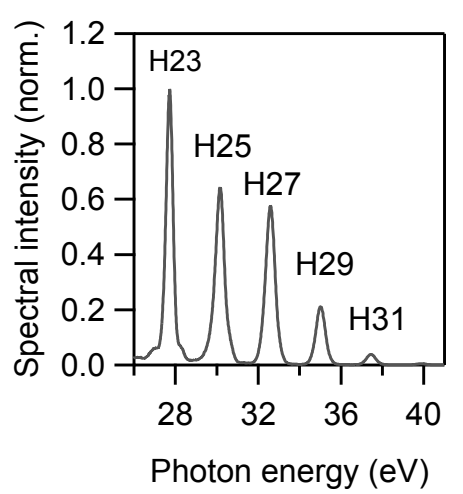

(b)

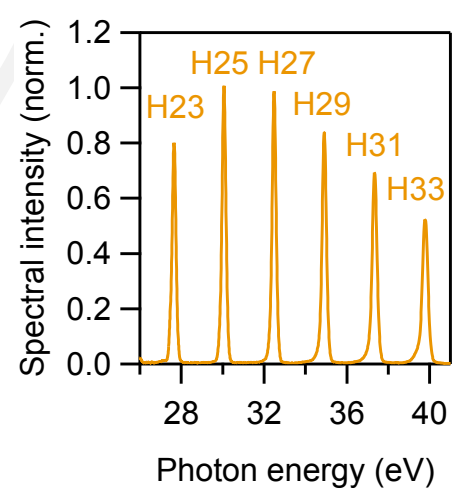

(c)

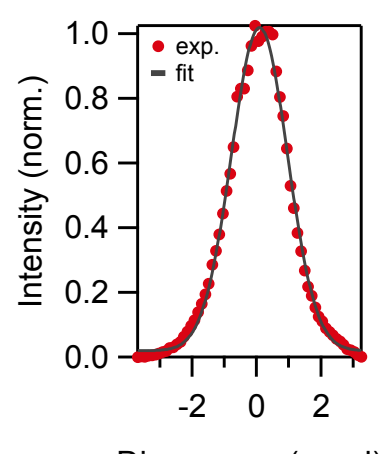

Figure 2.10. Typical HHG spectrum obtained with a) Xenon and b) Krypton as nonlinear medium. c) spatial far field beam profile of a single harmonic (Rothhardt et al., 2015). 
conditions and the driving laser pulse. A reduction of the bandwidth below $10^{-3}$ appears realistic. Such a XUV source is typically operated at $100 \mathrm{kHz}$ to $1 \mathrm{MHz}$ repetition rate and can be synchronized to the revolution frequency of ions in the storage ring. The XUV source is normally not tuneable in photon energy. However, the effective photon energy seen by the ions can be tuned via the ion velocity because of the Doppler shift. Even the moderate ion velocities at CRYRING allow for sufficient tuning of the effective excitation energy. For example, the mode H23 shown in Figure $2.11(27.7 \mathrm{eV})$ could be tuned across resonance 7,8,9 and 10 of $\mathrm{C}^{+}$shown in Figure 2.9. Later a selection of different harmonic orders with adequate filters in combination with Doppler tuning plus parallel and anti-parallel excitation would, in principle, allow for continuous scans of the excitation energy. A portable XUV source to be employed at the FAIR facility is currently under construction. It can be connected to CRYRING via a differential pumping section as illustrated in Figure 2.11.

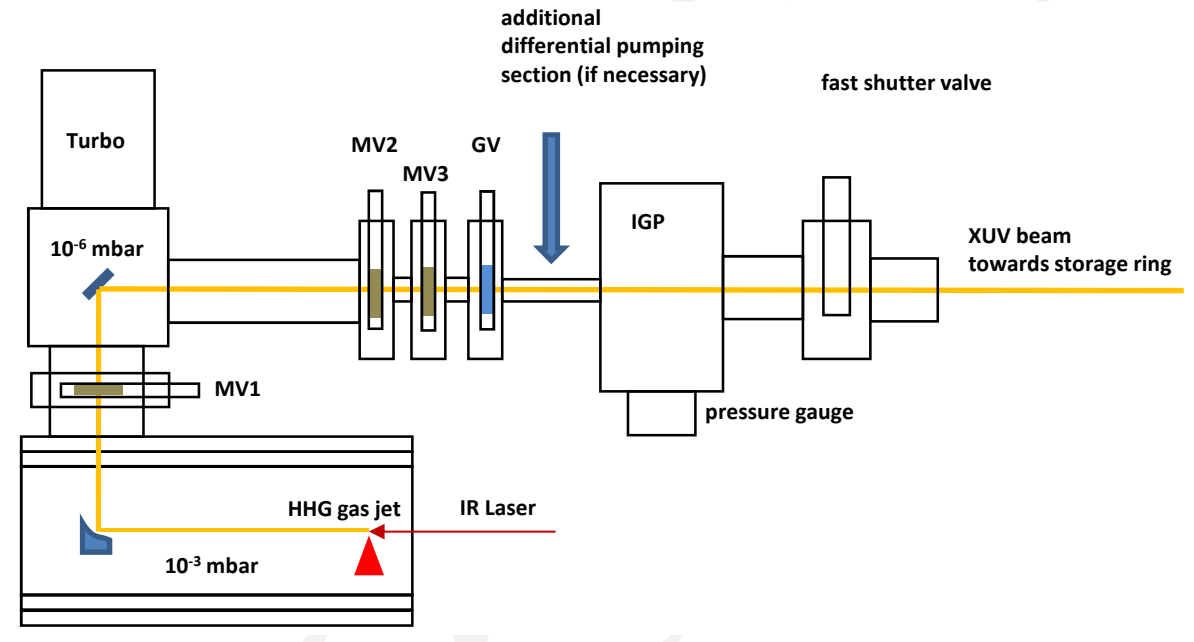

Figure 2.11. Schematic drawing of the XUV beam coupling section to the storage ring. Thin metal filter equipped valves (MV1-MV3) separate the different sections of the vacuum systems, turbomolecular pumps or ion-gether pumps will be employed for differential pumping to step-whise reduce the pressure to $<10^{-9}$ mbar. Fast shutter valves can separate the XUV source from the storage ring in case of broken filters or vacuum leaks.

The $\mathrm{C}^{+}$proof-of-principle experiment will demonstrate the feasibility of our new approach. As the XUV sources mature such experiments have the potential to become superior to the traditional singlepass photon-ion merged-beams approach since ion storage allows for the preparation of pure ground-level ion beams. In addition, resonant photoionization provides access to the atomic structure of ions, in particular, to multiply excited states. In this respect, resonant photoionization is complementary to electron-ion collision spectroscopy (Section 2.1.3 and 3.1) where multiply excited resonance states are formed during photorecombination, i.e., by the time-inverse process of photoionization. As laser technology progresses higher photon energies might be reached as well as photon energy spreads smaller than what is available for electron beams. This would enable the experimental characterization of resonance states in highly charged ions with hitherto unattainable precision. Such measurements would be of utmost interest, e.g., for the study of isotope effects with exotic nuclei from the FAIR accelerators. 


\subsection{Atomic collisions dynamics}

\subsubsection{Magnetic interaction and retardation effects upon the electron emission from HCI}

Studies of X-ray emission from HCIs have been found to be a unique tool for improving our understanding of the electron-electron (e-e) and electron-photon interactions in the presence of strong fields (Fritzsche et al., 2005, 2009). The x-ray spectroscopy of these systems showed that accurate energies and cross sections are obtained only if, apart from the static Coulomb repulsion among the electrons, the magnetic and retardation contributions to the electron-electron interaction as well as the leading quantum-electrodynamical effects are taken into account (Artemyev et al., 2013; Yerokhin et al., 2014; Fritzsche et al., 2015). In contrast to the x-ray spectroscopy, however, much less is known so far about how the magnetic and retarded interaction among the electrons affect their emission and, hence, the dynamics of electrons in strong fields.

To gain further insight into the electron dynamics, the excitation and autoionization of highly charged ions has been re-analyzed with the aim to separate the magnetic and retardation contributions to the electron-electron (e-e) interaction from the static Coulomb repulsion in strong fields. A remarkable change in the electron angular distribution due to the relativistic terms in the e-e interaction is found especially for the autoionization of (initially) beryllium-like projectiles, following a $1 s \rightarrow 2 p_{3 / 2}$ Coulomb excitation in collision with some target nuclei. In this process, the angular distribution of the emitted electron is given by

$$
W(\theta) \propto 1+\sum_{k=2,4, \ldots} \mathscr{A}_{k}\left(\alpha_{r} J_{r}\right) f_{k}\left(\alpha_{r} J_{r}, \alpha_{f} J_{f}\right) P_{k}(\cos \theta)
$$

where $\mathscr{A}_{k}\left(\alpha_{r} J_{r}\right)$ characterizes the alignment of the intermediate state after the excitation, $\theta$ is the polar angle with regard to the beam direction and where the $f_{k}\left(\alpha_{r} J_{r}, \alpha_{f} J_{f}\right)$ are characteristic functions that describe the dynamics of the autoionization. The function $f_{k}$ in Equation 2.3 merely depends on the (reduced) matrix elements $\left\langle\left(\alpha_{f} J_{f}, \varepsilon \kappa\right) J_{r}\|V\| \alpha_{r} J_{r}\right\rangle$ of the (frequency-dependent) e-e interaction

$$
\begin{aligned}
V & =V^{\mathrm{C}}+V^{\mathrm{B}} \\
& =\sum_{i<j}\left(\frac{1}{r_{i j}}-\left(\boldsymbol{\alpha}_{i} \cdot \boldsymbol{\alpha}_{j}\right) \frac{\cos \left(\omega r_{i j}\right)}{r_{i j}}+\left(\boldsymbol{\alpha}_{i} \cdot \nabla_{i}\right)\left(\boldsymbol{\alpha}_{j} \cdot \nabla_{j}\right) \frac{\cos \left(\omega r_{i j}\right)-1}{\omega^{2} r_{i j}}\right)
\end{aligned}
$$
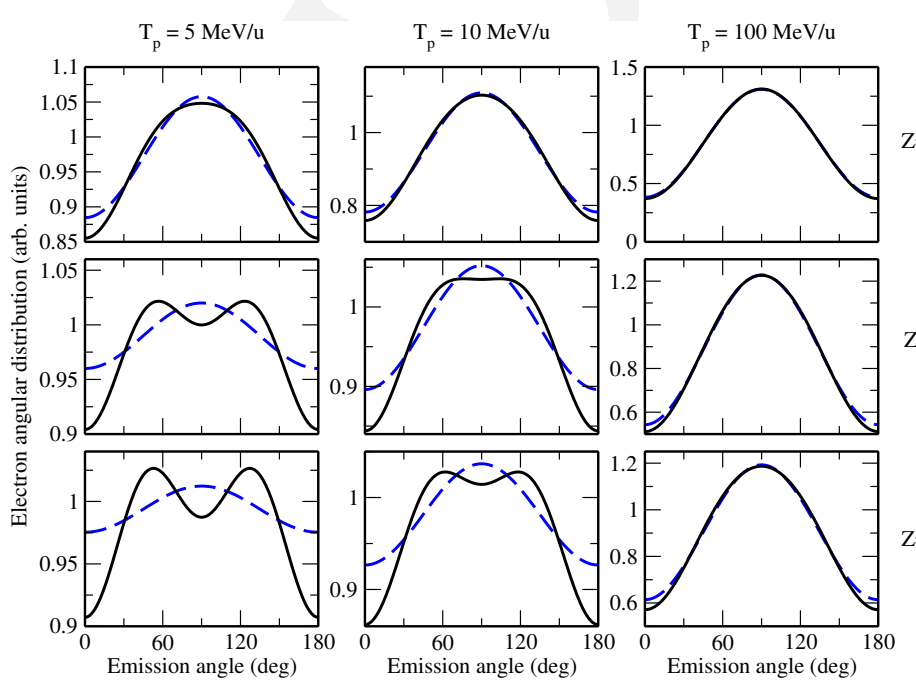

Figure 2.12. Angular distributions of electrons emitted in the $1 s 2 s^{2} 2 p_{3 / 2}{ }^{3} P_{2}-$ $1 s^{2} 2 s^{2} S_{1 / 2}$ autoionization of beryllium-like ions with projectile energies $T_{p}=5,10$ and $100 \mathrm{MeV} / \mathrm{u}$, following the Coulomb excitation from their $1 s^{2} 2 s^{2}{ }^{1} S_{0}$ ground state. Results are shown in the rest frame of three beryllium-like projectiles with charges $Z=$ 54, 79 and 92 and in two approximations. Angular distributions with only the Coulomb repulsion incorporated into the Auger amplitudes (blue dashed lines) in Equation 2.3 are compared with those where the complete e-e interaction is taken into account (black solid lines). 
Figure 2.13. Angular distributions of electrons emitted in the $1 s 2 s^{2} 2 p_{3 / 2}{ }^{3} P_{2}-$ $1 s^{2} 2 s^{2} S_{1 / 2}$ (left panel) and $1 s 2 s^{2} 2 p_{3 / 2}{ }^{3} P_{2}-$ $1 s^{2} 2 p^{2} P_{1 / 2}$ (right panel) autoionization of $\mathrm{U}^{88+}$ projectiles with energy $T_{p}=5 \mathrm{MeV} / \mathrm{u}$. Results are shown in the laboratory frame and by incorporating only the Coulomb repulsion into the Auger amplitude (blue dashed lines) in Equation 2.3 as well as for a full account of the e-e interaction (black solid lines).
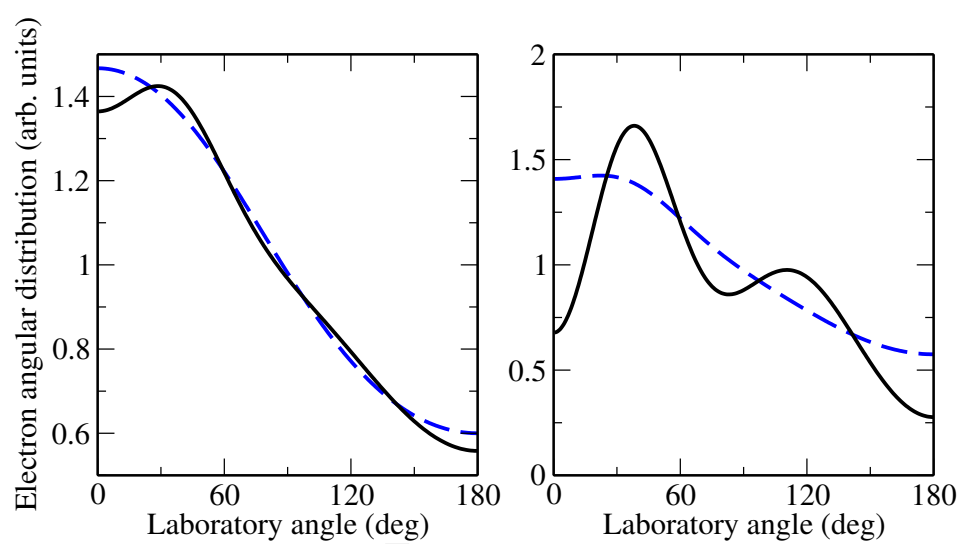

that comprises both, the instantaneous Coulomb repulsion (first term) and the Breit interaction, i.e. the magnetic and retardation contributions (second and third term).

For this excitation-autoionization process via the $1 s 2 s^{2} 2 p_{3 / 2}{ }^{3} P_{2}$ resonance, a diminished (electron) emission in forward direction as well as oscillations in the electron angular distribution due to the magnetic and retarded interactions are predicted especially for the Auger decay into the $1 s^{2} 2 s^{2} S_{1 / 2}$ ground and $1 s^{2} 2 p^{2} P_{1 / 2}$ excited levels of the finally lithium-like ions. This emission pattern is in strong contrast to a pure Coulomb repulsion between the bound and the outgoing electrons. For example, Figure 2.12 displays the angular distribution for the autoionization of the $1 s 2 s^{2} 2 p_{3 / 2}{ }^{3} P_{2}$ resonance into the $1 s^{2} 2 s^{2} S_{1 / 2}$ ground state of the lithium-like ion for three projectile energies $T_{p}=5,10$ and $100 \mathrm{MeV} / \mathrm{u}$ and for beryllium-like ions with nuclear charge $Z=54,79$ and 92 . The angular distributions of the corresponding electron lines is calculated in two approximations with regard to how the e-e interaction is included into the characteristic functions $f_{k}$. Apart from a pure Coulomb repulsion between the bound and outgoing electrons (blue dashed lines), we here consider the full relativistic e-e interaction (Equation 2.4) in this figure (black solid lines). A rather strong interference between the Coulomb and the magnetic terms in the e-e interaction arises especially at low projectile energies, $T_{p} \lesssim 10 \mathrm{MeV} / \mathrm{u}$, and gives rise to a double-peak structure in the angular distributions as well as to a $10 \%$ reduction of the electron yield in forward direction if the nuclear charge of the projectiles is increased from $Z=54$ to 92 . This lowering of the electron emission in forward direction $\left(\theta \approx 0^{\circ}\right)$ is significant and further enhanced in the laboratory frame due to the Lorentz transformation of the energetic electrons, cf. Figure 2.13.

In conclusion, the proposed excitation-autoionization process can be observed at existing storage rings and will provide novel insight into the dynamics of electrons in strong fields (Fritzsche et al., 2012). The most simple signatures of the relativistic contributions to the e-e interaction in high- $Z$ ions is the reduced electron emission in forward direction $\left(\theta \lesssim 5^{\circ}\right)$ as well as the double-peak structure in the expected angular distribution; these signatures arise especially at low projectile energies $\lesssim 10 \mathrm{MeV} / \mathrm{u}$ and for beryllium-like ions with nuclear charge $Z \gtrsim 70$. The electron angular distribution from such projectiles can be analyzed with present-day electron spectrometers and provide complementary information about the electron dynamics in strong fields that is not accessible from x-ray spectra alone.

\subsubsection{Inelastic Electron Scattering in Inverse Kinematics: Spectroscopy and Colli- sion Dynamics at CRYRING and ESR}

In atomic collision physics, $(e, 2 e)$-spectroscopy has itself established as one gold standard and the most significant test of theory for determination of atomic structure, e.g. state specific momentum wave func- 


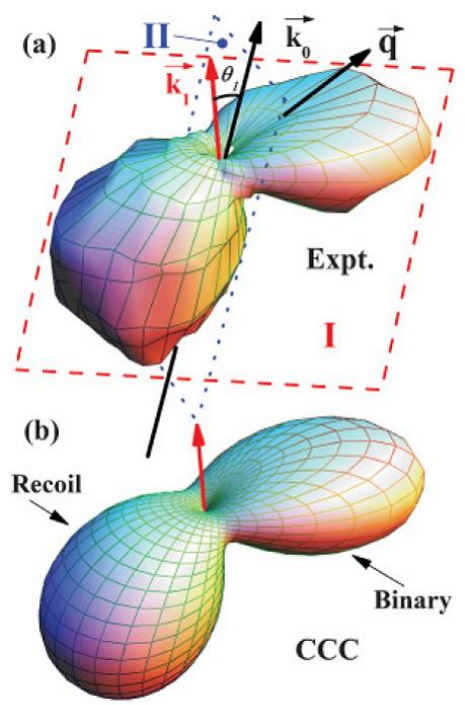

Figure 2.14. Absolute TDCS for $(e, 2 e)$ on He for incident energy $E_{0}=70.6 \mathrm{eV}$, i.e. an excess energy of $46 \mathrm{eV}$ above threshold (Ren et al., 2011a,b, 2010, 2012). The TDCS is displayed as a function of the emission angle of an electron with kinetic energy of $E_{2}=5 \mathrm{eV}$. The emission angle of the other electron $\left(E_{1}=41 \mathrm{eV}\right)$ is fixed to $\Theta_{1}=-20^{\circ}$ (a) Experimental 3D cross section. (b) CCC calculation. The dashed and dotted frames in (a) indicate the cutting planes through the 3D pattern in the scattering (I) and perpendicular plane (II), respectively (for details see Ren et al., 2011a,b, 2010, 2012).

tions, and for the quantitative description of collision dynamics on the atomic scale. The applied experimental techniques, however, are limited to using neutral atomic and molecular targets and are not practically applicable for corresponding studies with ions (McCarthy and Weigold, 1991). In this context we are developing in-ring experiments in CRYRING and ESR to measure kinematically complete triple differential cross sections (TDCS) $\frac{d^{3} \sigma}{d \Omega_{1} d \Omega_{2} d E_{2}}$ for $(e, 2 e)$ electron impact ionization of H-, He-, $\mathrm{Li}$-like and other few-electron ions over a wide $Z$ range and for selected ions of astrophysical relevance,

$$
e^{-}\left(E_{0}\right)+A^{q+} \rightarrow A^{(q+1)+}+e_{\text {fast }}^{-}\left(E_{1}, \theta_{1}\right)+e_{\text {slow }}^{-}\left(E_{2}, \theta_{2}\right)
$$

We have shown, that kinematically complete collisions of the type Equation 2.6 can be very efficiently investigated via inverse kinematic reactions, e.g., for He-like configurations (Kollmus et al., 2002),

$$
A^{q+}\left(1 \mathrm{~s}^{2}\right)+\mathrm{He} \rightarrow A^{(q+1)+}(1 \mathrm{~s})+\mathrm{He}^{+*}+e_{\text {fast }}^{-}\left(E_{a}^{L}, \theta_{a}\right)+e_{\text {slow }}^{-}\left(E_{b}^{L}, \theta_{b}\right) .
$$

Here, the cold He-target produced by an ultrasonic jet serves as a source of quasi-free electrons. A collision according to Equation 2.7 with a specific energy $E_{\mathrm{i}}$ of the incident heavy ion $A^{q+}$ corresponds to an electron impact ionization according to Equation 2.6 with the same collision velocity, when the incident electron collision energy $E_{0}=E_{\mathrm{i}} / \frac{u}{m_{e}} \approx E_{\mathrm{i}} / 1823$.

The $(e, 2 e)$ experiments devised for the CRYRING@ESR storage-ring combination thus permit to cover the TDCS along the isoelectronic sequences of few-electron high- $Z$ ionic targets for a wide range of charge states. This allows to extend these studies well into the relativistic domain. The kinematically complete $(e, 2 e) 1$ s-ionization of ions with very high $Z$ up to 92 will be preferably studied with emphasis on $\mathrm{H}-, \mathrm{He}-$ and Li-like ions. The collision energy range provided by CRYRING makes this storage ring particularly suited to focus on the theoretically very important region just above the ionization threshold: for kinematically complete 1 s-electron impact ionization this applies for intermediate- $Z$ ions up to $Z=36$ and for 2 s- and 2p-ionisation for ions up to $Z=70$ and 74, respectively. For M-shell ionization, all ions up to uranium may be covered by the collision energy ranges available at CRYRING.

These $(e, 2 e)$ experiments constitute critical tests of advanced ab-initio theories exterior complex scaling (ECS) and covergent close coupling (CCC) for highly charged ionic targets (Schubert et al., 1979; Ehrhardt et al., 1971; Dürr et al., 2007; Bray et al., 2012). The configuration of a heavy ion storage 
ring combined with inverse kinematics opens up unique experimental possibilities. The achievable luminosities exceed by many orders of magnitude those available from conventional electron-ion crossedbeams arrangements (which are superior for very high resolution spectroscopic studies, see Section 5.4). High luminosities are a prerequisite, in particular, for kinematically complete ionization experiments on high-Z HCI.

It is important to note that $(e, 2 e)$ experiments with ions (in contrast to those with neutral atoms) which measure the TDCS for inner shell ionization (as opposed to outer shell ionization) access highly critical configurations for ionization: applying inverse kinematics using HCI with vacant outer shells, e.g., the $1 s$ ionization investigated in $\mathrm{Au}^{77+}$ instead of $1 s$ ionization in neutral $\mathrm{Au}\left(\mathrm{or} \mathrm{Au}^{1+}\right.$ ) is the only way to remove essential ambiguities arising from multiple outer shell ionization in measurements of TDCS for inner shell ionization (Keller and Dreizler, 1998; Keller et al., 1999).

The narrow impact energy range of validity of the Wannier theory above the ionization threshold (typically few $\mathrm{eV}$ at low electron collision energies) has been investigated in detail for $\mathrm{H}$ and $\mathrm{He}$ targets, but for ions in various charge states the range of validity is still open to debate, particularly when the $Z$-dependence is considered for HCI. This appears plausible under the following considerations: The momentum transfer vector $\vec{q}$ which is very meaningful at collision energies well above threshold because it provides an orientation axis for most of the emitted electrons, loses its relevance as a single determining parameter close to threshold (see Figure 2.14 for an intermediate collision energy region, where already the direction of $\vec{q}$ deviates noticeably from the center of the lobe characterizing the TDCS) (Ren et al., 2010, 2011a,b). This axis corresponds to the direct ionization amplitude (Ehrhardt et al., 1986) but is meaningless for the other ionization amplitudes (exchange and resonance/capture) which close to threshold in the Wannier region have magnitudes comparable to the direct amplitude. Here data for $(e, 2 e)$ ionization along isoelectronic sequences are needed for a rigorous quantitative understanding of the collision dynamics.

The tremendous success of a-priori theories like ECS and CCC in correctly producing the full TDCS for $\mathrm{H}$ and $\mathrm{He}$ (see for example Figure 2.14) over the entire collision energy range had given rise to hopes for a final theoretical solution of impact ionization (Bray et al., 2010). However, it was found that the validity of these two most advanced theories at this time is indeed restricted to bound $s$-states and $\mathrm{H}$ and He targets. Already for initial $p$ states of Ne they fail to reproduce the experimental TDCS cross sections. It is thus of utmost importance to provide experimental benchmarks for TDCS for electrons from several inner shells, for $1 s$ and $2 s$ ionization for ions of the $\mathrm{H}, \mathrm{He}$, and $\mathrm{Li}$ isoelectronic sequences for medium and high $\mathrm{Z}$ as well as for the $\mathrm{Ne}$ and $\mathrm{Ar}$ isoelectronic sequences for closed $p$-shells and for the $\mathrm{B}$ and $\mathrm{C}$ isoelectronic sequences for one and two-electron open $p$ shells.

In Figure 2.15 we show distorted wave Born approximation (DWBA) calculations which were performed using the code of McCarthy (1995) for TDCS for electrons of $E_{0}=16457 \mathrm{eV}$ ionizing the $1 \mathrm{~s}$ electron of $\mathrm{Fe}^{24+}$ (equivalent to $30 \mathrm{AMeV} \mathrm{Fe}^{24+}+\mathrm{He}$ ). The angular distribution of the ionized electrons is strongly dependent on the scattering angle of the incident electron, i.e., the momentum-transfer vector $\vec{q}$. These angular distributions, when transformed from the projectile frame (= emitter frame) into the laboratory frame, are the distributions to be found on the 2D-position sensitive electron detector in the focal plane of the forward electron spectrometer. The peculiarities of the features observed in the TDCS for high- $Z$ atomic targets and attributed to relativistic terms (Keller and Dreizler, 1998; Keller et al., 1999) can then for highly charged ions be traced quantitatively without ambiguities due to many-electron effects in neutral atoms. A comparison of these TDCS for ions with those for neutral atoms then allows to 


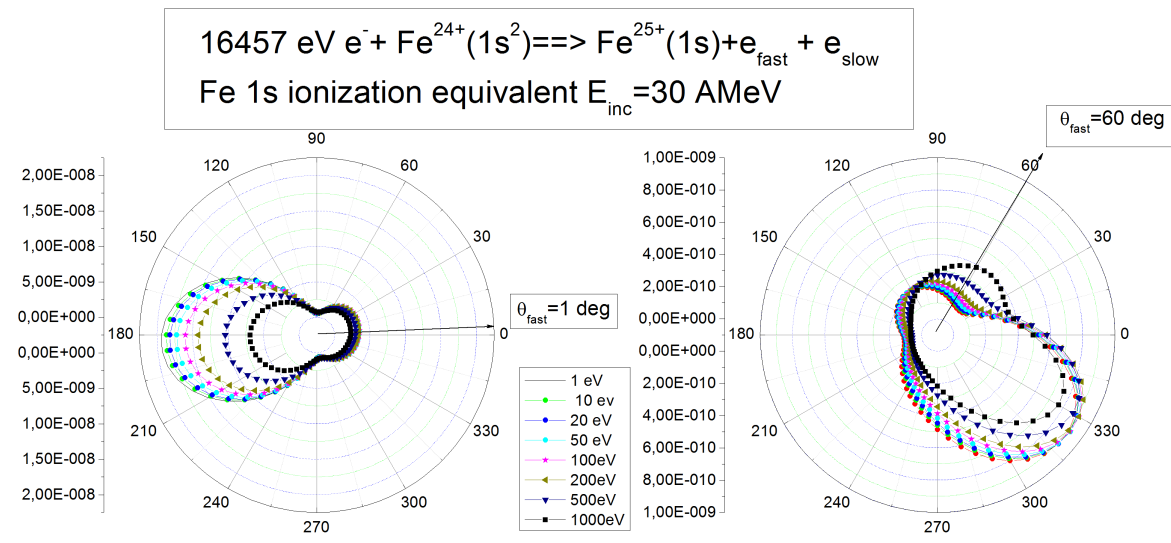

Figure 2.15. TDCS for electron impact ionization of He-like $\mathrm{Fe}^{24+}\left(1 \mathrm{~s}^{2}\right)$ for $16457 \mathrm{eV}$ impact energy in the projectile frame corresponding to a laboratory collision energy $30 \mathrm{AMeV}$. Calculations were performed with the DWBA code of McCarthy (1995). The momentum transfer to the ionizing projectile is 11 a.u. for 1 degree scattering angle and 32 a.u. for 60 degree scattering angle.

quantitatively assess the role of the different mechanisms identified by Keller and Dreizler (1998); Keller et al. (1999). Interestingly enough, kinematically complete experiments for $1 s$ ionization very close to threshold will also permit high precision measurements of the $1 s$ binding energy due to the kinematic relations of ionized electron energies in the emitter and laboratory frames (Ullrich et al., 1996).

For $1 s$ electron-impact ionization of $\mathrm{Fe}$ with a $16.95 \mathrm{AMeV} \mathrm{Fe}^{25+}$ beam corresponding to an incident electron energy of $20 \mathrm{eV}$ above the $1 s$-ionization threshold Figure 2.16 shows the laboratory energy- and angular distribution of the inelastically scattered electrons in the continuum. It is apparent that using the $(e, 2 e)$ technique in inverse kinematics current high energy resolution forward electron spectrometers permit determination of the $1 s$ binding energy, e.g., in the $\mathrm{H}$-like $\mathrm{Fe}^{25+}$ projectile, competitive with those achieved by photoionization. Experiments will be performed with a longitudinal reaction microscope (Ullrich et al., 2003) combined with the existing 2D- position sensitive in-ring forward electron spectrometer in ESR and a new 2D- position sensitive forward electron spectrometer in CRYRING.

\subsubsection{Radiative electron capture to the continuum and the high-energy endpoint of electron-nucleus bremsstrahlung}

It has been shown previously that storage rings offer a supremely well suited environment, where the fundamental process of electron-nucleus bremsstrahlung can be studied in inverse kinematics in kinematically complete fashion at the theoretically important high-energy endpoint of the bremsstrahlung: bremsstrahlung photons from the high-energy endpoint appear in coincidence with electrons emitted under $0^{\circ}$ with respect to the projectile beam in the radiative electron capture to the continuum (RECC) cusp (Nofal et al., 2007; Hillenbrand et al., 2014a). For this highly interesting region, theory has postulated deep lying equivalences of electron-nucleus bremsstrahlung, photoionization, and radiative recombination. In the rest-frame of the nucleus, all energy is transferred from the incoming electron to the emitted bremsstrahlung photon, such that the outgoing electron has (almost) no kinetic energy with respect to the nucleus. This case cannot be investigated in classical configurations of electron beams impinging on a target at rest because of the practical impossibility of detecting an electron, which leaves the target without kinetic energy. 


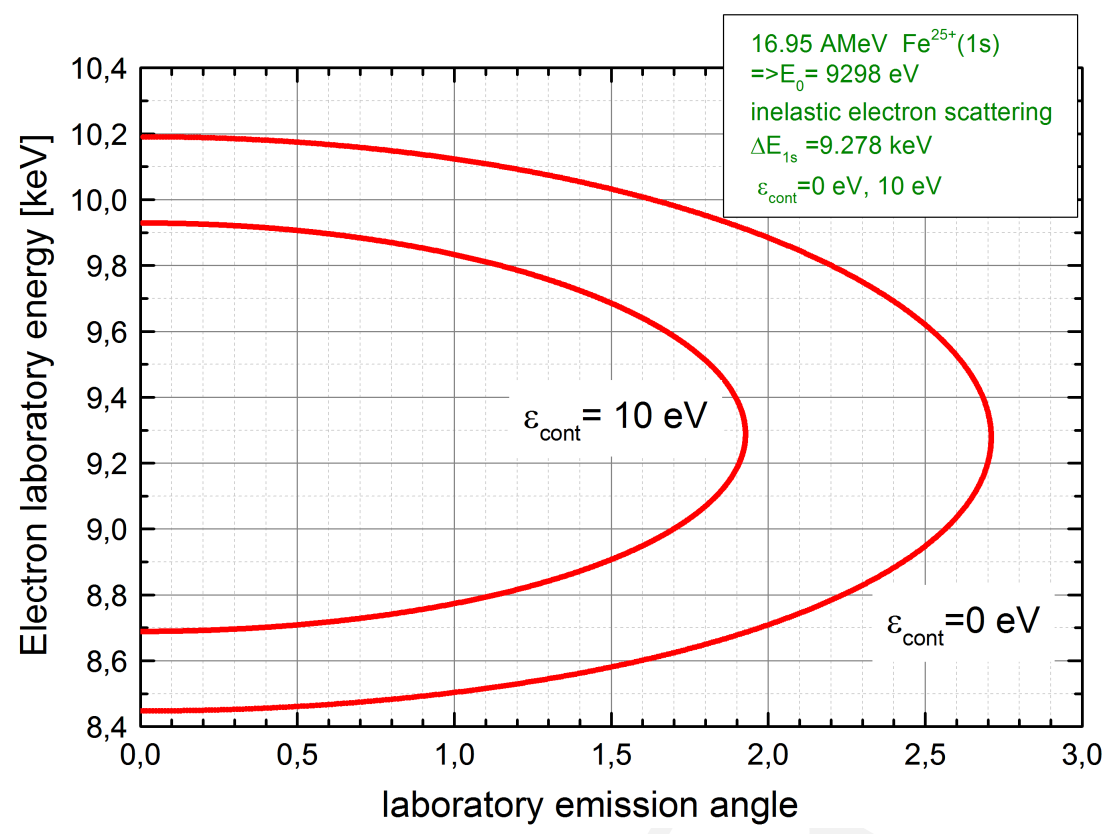

Figure 2.16. Laboratory energy of inelastically scattered electron after ionizing $\mathrm{H}$-like $\mathrm{Fe}^{25+}$ as function of laboratory observation angle.

Figure 2.17. Radiative-electron-capture-to-continuum cusp for the process $\mathrm{U}^{88+}+\mathrm{N}_{2} \rightarrow \mathrm{U}^{88+}+\left[N_{2}^{+}\right]^{*}+$ $e^{-}\left(E_{f}, \vartheta_{f}=0^{\circ}\right)+\gamma\left(\vartheta_{\gamma}=90^{\circ}\right)$ at a collision energy of $90 \mathrm{MeV} / \mathrm{u}$ as a function of the electron emission energy $E_{f}$ : The experimental results (blue dots) are compared with theoretical calculations applying fully relativistic Dirac wave functions (red solid line) and applying semi-relativistic Sommerfeld-Maue wave functions multiplied by 2 (green dashed line). Results taken from Hillenbrand et al. (2014a)

electron energy in projectile system $E_{f}^{\prime}[\mathrm{keV}]$

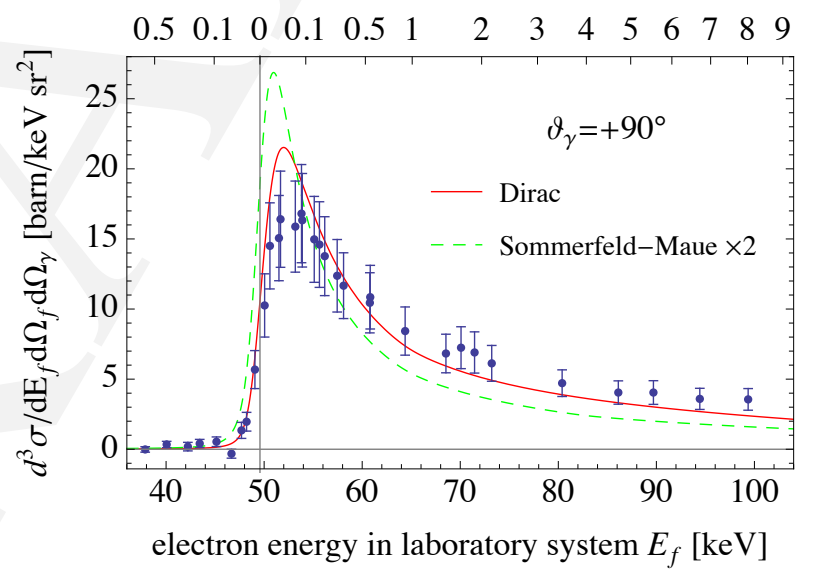

The previous experiment of Hillenbrand et al. (2014a) was performed at the ESR for the collision system $\mathrm{U}^{88+}+\mathrm{N}_{2}$ at a projectile energy of $90 \mathrm{MeV} / \mathrm{u}$. Using a magnetic electron spectrometer and appropriate photon detectors, the energy distribution of electrons emitted under $0^{\circ}$ was measured in coincidence with the angular distribution of bremsstrahlung photons. The results were shown to be in agreement with fully relativistic calculations using Dirac wave functions while refuting the applicability of semi-relativistic Sommerfeld-Maue wave functions for this process (Figure 2.17). The interest of extending this study towards lower collision energies is twofold. Firstly, the RECC cross section increases with decreasing collision velocity approximately as $v_{p}^{-5}$. The associated increase of the observable count rate permits to study the process in finer detail. Secondly, the asymmetry of the RECC cusp, which is seen in Figure 2.17, increases with decreasing collision velocity approximately as $Z_{p} / \gamma v_{p}$ (Jakubassa-Amundsen, 2003), thus making studies of RECC with high projectile charges $Z_{p}$ at low collision velocities most fruitful.

As was predicted theoretically by Jakubassa-Amundsen (2003) and confirmed experimentally by 
Hillenbrand et al. (2015), RECC is competing with the non-radiative electron capture to the continuum (ECC) process where an initial target electron is transferred to the projectile continuum. It was shown that the cross sections of both processes are comparably large for a nitrogen target at $90 \mathrm{MeV} / \mathrm{u}$. Going to lower collision velocities, the collision energy at which ECC and RECC are comparably large, was predicted by Jakubassa-Amundsen (2007) to be around $11 \mathrm{MeV} / \mathrm{u}$ for a hydrogen target, independent of the projectile charge $Z_{p}$. At CRYRING@ESR, where this collision energy is accessible, the previously performed studies on RECC and the high-energy endpoint of electron-nucleus bremsstrahlung can be extended to significantly increased levels of detail and precision. The experimental data will thus provide further stringent tests to most advanced theories of photon-matter interaction, such as the fully relativistic Dirac theory of bremsstrahlung (Müller et al., 2014).

\subsubsection{Complete Atomic Fragmentation with Topologically Stable Multi-electron Trans- fer into the Projectile Continuum}

The future storage ring CRYRING@ESR provides the unique opportunities to store highly charged ions of very high- $Z$ bare $\mathrm{U}^{92+}$ and $\mathrm{H}$-like $\mathrm{U}^{91+}$ at low energies in the $1-10 \mathrm{MeV} / \mathrm{u}$ range. Using the combination of the highest ion charge state $q=92$ with low collision velocities such as $v=6$ a.u., a qualitatively new collision regime will be accessible: The large Sommerfeld parameter $s=q / v \approx 15$ indicates a regime dominated by very strong perturbations, and the low collision velocity further indicates highly adiabatic collisions, at least when considering inner shell processes for heavy ions. At present there is no ab-initio theory available that reliably describes the ionization dynamics in this collision regime. This can be attributed to the fact that the observed multiple ionization is as strong as single ionization. It neither follows simple binomial statistics nor can it be described by perturbation theory. Actually, perturbation theory is not applicable for $s>1$ (Åberg, 1991), and that multiple-ionization distributions here The complications encountered by current theories can to a large extent be attributed to the fundamental difficulty to describe the infinite-range multi-electron Coulomb continuum correctly. So far, theory has failed to quantitatively reproduce the experimental results (Lahmam-Bennani et al., 2002, 2010; Jia et al., 2002).

Experiments in adiabatic, but moderate perturbation conditions $s \geq 1$ like $\mathrm{I}^{23+}+\mathrm{He}$ at $0.35 \mathrm{MeV} / \mathrm{u}$ have shown that in the ionization a topologically stable multi-electron transfer into the projectile continuum dominates (compare Figure 2.18a,b). This is entirely at variance with observations in ionizing collisions of fast ions with atoms at small perturbations $s \ll 1$, e.g. $\mathrm{p}+\mathrm{Ne}$, that single and sparse multiple electron continua are dominated by electrons being removed from the target atom with minimum momentum transfer $q=\Delta E / \hbar v_{\text {proj }}$ into the continuum. The overwhelming number of electrons for small perturbations $s$ is found with low kinetic energies near threshold in the laboratory (slow electron emission (SEE) target cusp at $v_{e} \approx 0$ in Figure 2.18a).

For slow adiabatic collisions with a large Sommerfeld parameter $s$ the multi-electron continuum is dominated by a singularly sharp ECC electron cusp at $v_{e}=v_{\text {proj }}$ (Figure 2.18b), whose half width even decreases with increasing projectile charge. This indicates that some new propensity rule may be active which - one may speculate - leads phenomenologically to a near conservation of the nodes in the electron density profile during transfer. This observation and closely related results from very heavy projectile-recoil coincidence-experiments (Datz et al., 1990) can presently not be described by theory. For CRYRING@ESR, it is intended to investigate in adiabatic collisions of HCI, like $\mathrm{Au}^{79+}$ 


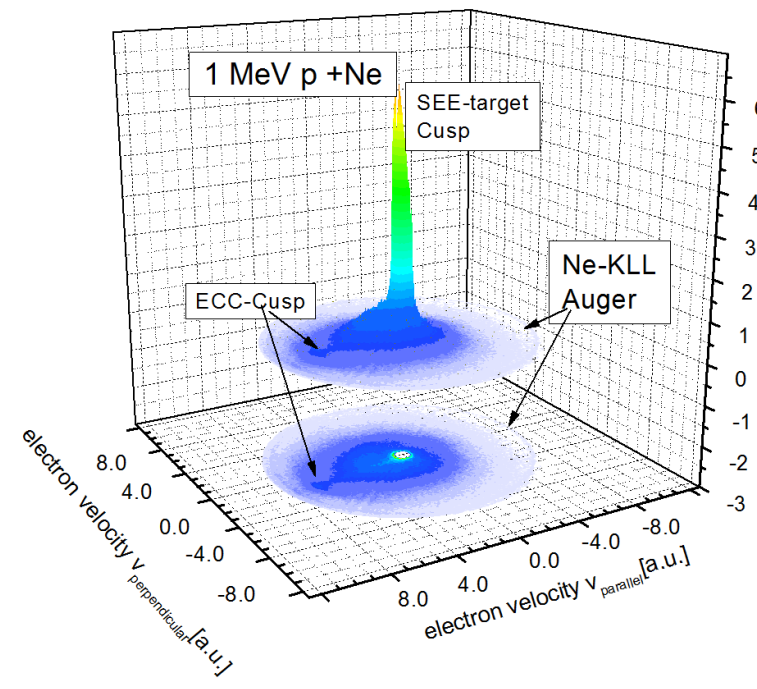

(a)

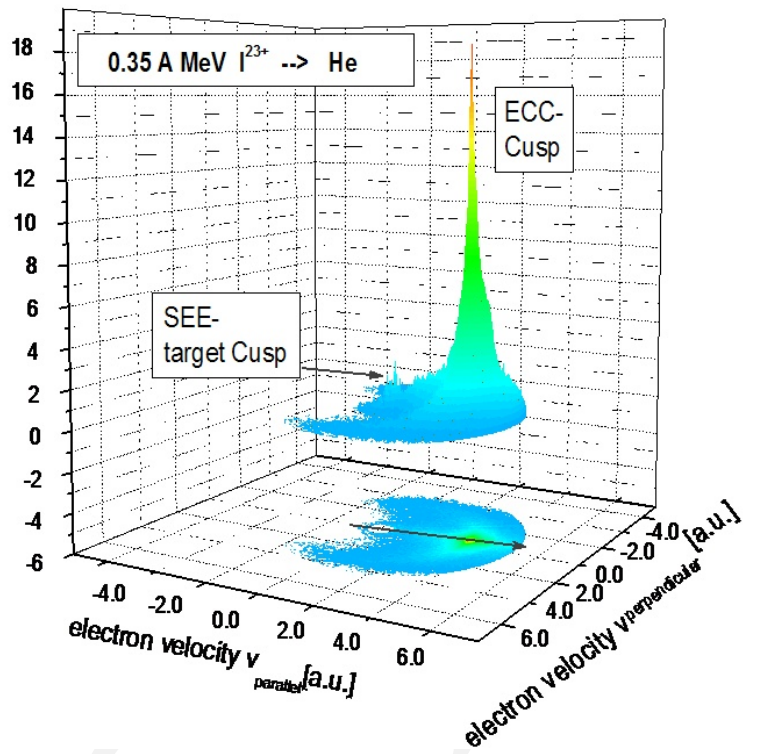

(b)

Figure 2.18. (a) Double differential cross section for electron emission in 1-MeV p+Ne collisions (Hagmann et al.). The prominent features for this small-perturbation collision are the $\mathrm{SEE}$ cusp near $0 \mathrm{eV}$ in the lab frame, the small but perceptible ECC cusp and the ring of Ne-KLL Auger electrons.

(b) Double differential cross section for electron emission in $0.35-\mathrm{MeV} / \mathrm{u} \mathrm{I}^{23+}+\mathrm{He}$ collisions (Hagmann

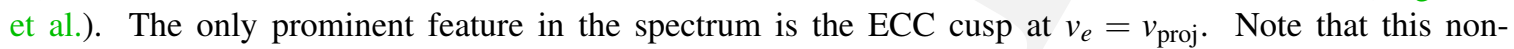
coincident spectrum is dominated by the contributions from multiple ionization of the target. The SEE cusp at threshold in the laboratory around $v_{e}=0$, which is the most prominent feature in fast collisions and for small perturbation $s$ like $\mathrm{p}+\mathrm{Ne}$ is marginal in these high-perturbation adiabatic collisions like $\mathrm{I}^{23+}+\mathrm{He}$.

and $\mathrm{U}^{91+, 92+}$, the electron continua corresponding to multiple ionization up to complete atomic fragmentation with a reaction microscope and the forward electron spectrometer. An example for such an experiment could be

$$
5-\mathrm{MeV} / \mathrm{u}-\mathrm{U}^{91+}+\mathrm{Ar} \rightarrow \mathrm{U}^{(91-q)+}+\mathrm{Ar}^{n+}+(n-q) e^{-}+Q .
$$

The final vector momenta of all ionized electrons will be determined in complete kinematics with particular emphasis on the momentum-transfer dependence of the topologically stable multiple ionization resulting in the sharp electron cusps which dominate the electron distribution in the continuum. It has been found that in such highly adiabatic collisions of highly charged ions (Datz et al., 1990; Hagmann et al.) simultaneously with ionization a large number of electrons is transferred into bound states of the projectile. For understanding this kind of collisions it is therefore important to elucidate the interdependence of continuum energy and bound final $(n, l)$ state distribution in dependence on multiplicity of capture, $q$, and of ionization, $n$, respectively, and endo-/exoergicity $Q$. These experiments are expected to provide benchmarks for advanced ab-initio theories for describing the apparently highly structured multi-electron continua generated in strongly perturbing adiabatic collisions.

\subsubsection{Precision studies of Ion-Lithium collisions with a MOTReMi in CRYRING}

Ion-impact induced ionization of atoms or molecules provides a very well suited testing ground for the investigation of the dynamics of correlated few-particle systems. This is largely due to the very good understanding that was achieved already about the underlying interaction, i.e. the electromagnetic field, 


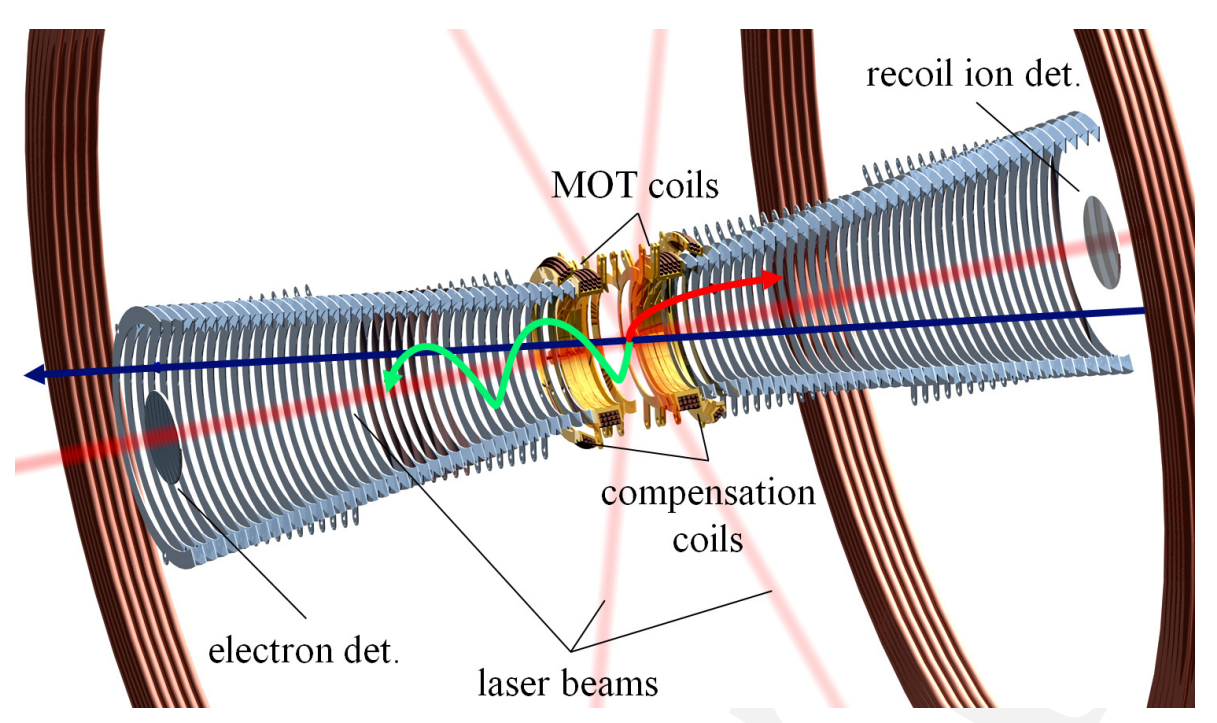

Figure 2.19. The MOTReMi apparatus. Electron, target ion, and projectile trajectories are indicated by the green, red, and dark-blue lines, respectively.

acting between pairs of particles. However, solving the equations of motion is by no means trivial as soon as more than two particles are involved. This is known as the 'few-particle problem' which is one of the most fundamental and, at the same time, challenging theoretical problems in physics (e.g. Rescigno et al., 1999; Bray, 2002; Feist et al., 2008; Guan et al., 2008). Highly charged ions represent a particularly interesting class of projectiles as the interaction strengths can be varied from a photon-like perturbative regime to a strongly non-perturbative region at very small velocities which e.g. for electrons would bring the collision energy below the ionization threshold. Interaction times from femtoseconds down to zeptoseconds in relativistic collisions can be realized corresponding to the shortest, and for very high projectile charges most intense electro-magnetic pulses $\left(>10^{22} \mathrm{~W} \mathrm{~cm}^{-2}\right)$ that are accessible in laboratories. Thus, ion collisions provide benchmarks for theoretical models covering very 'exotic' situations in extremely strong fields (McGovern et al., 2010; Fischer et al., 2003b).

On the experimental side, the most detailed information on ionization reactions can be obtained in kinematically complete experiments, i.e. in studies where the momenta of all involved particles are analyzed in coincidence. For electron or photon impact, such experiments are routinely performed since the 1960s measuring the energy and angular distributions of the free electrons in the final state. However, obtaining highly differential data for ion-atom collisions is much more involved, because the projectile scattering angles and, therefore, the relative projectile momentum changes, are often immeasurable small (at large projectile energies typically $10^{-6} \mathrm{rad}$ ). This became possible only about 20 years ago with the development of 'Reaction Microscopes', often also referred to as COLTRIMS (cold target recoil ion momentum spectroscopy) (e.g. Ullrich et al., 2003). Here, the momentum vectors of electrons and recoiling target ions are measured in coincidence and the projectile momentum change is calculated exploiting momentum conservation.

Recently, a next-generation Reaction Microscope was developed at the MPIK and operated in TSR (Fischer et al., 2012; LaForge et al., 2013; Hubele et al., 2013). In contrast to conventional Reaction Microscopes (ReMi), where the cold target is prepared by supersonic expansion in a gas jet, the novel apparatus uses a MOT for target preparation (Hubele et al., 2015). In this setup, which is referred to as MOT reaction microscope (MOTReMi) (see Figure 2.19), an atomic lithium target is cooled to temperatures of typically $\mathrm{mK}$, which allowed improving the achievable momentum resolution by a factor 
Figure 2.20. $Q$-value spectrum for single electron capture in $63 \mathrm{keV} \mathrm{Ne}^{7+}-\mathrm{He}$ collisions. The lower horizontal scale indicates the level energies in the $\mathrm{Ne}^{6+}$, the upper scale the $Q$-value of the reactions. The lines observed in the spectrum correspond to the electron capture in the $n=4$ subshells of the $\mathrm{Ne}^{6+}$ ion (Fischer et al., 2002).

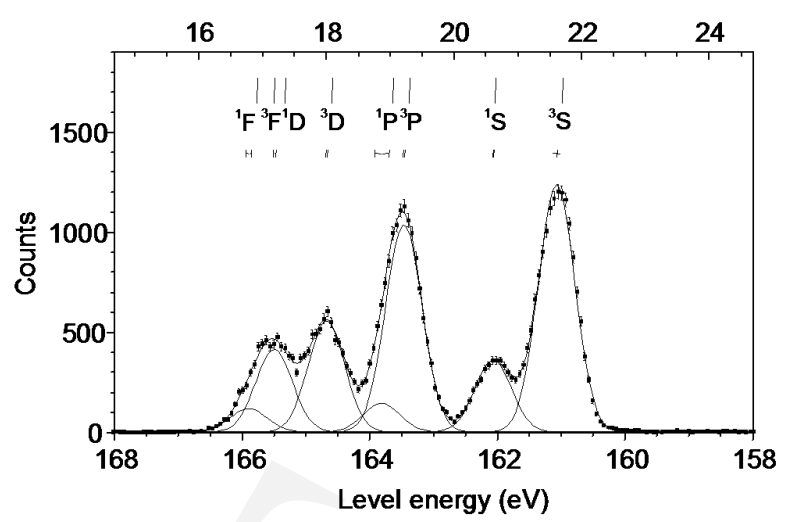

of 2 to 3 compared to conventional Reaction Microscopes. Extending the range of available targets to calcium is planned for the near future. CRYRING, in turn, offers a wide range of projectile species up to fully stripped ions with energies between 1 and $10 \mathrm{MeV} / \mathrm{u}$. That means collision systems covering a broad range of perturbations can be investigated. The reactions to be observed are ionization or excitation of the target or the projectile as well as charge transfer which also can occur in combination with each other. The experiments to be performed in the CRYRING will provide benchmark data in a regime where, if any, so far only limited or kinematically incomplete experimental data is available, and they will allow testing in detail the range of validity of standard theoretical methods and possibly even prompting theorists to develop new models. The high resolution achievable with the new momentum-imaging spectrometer will not only allow to study the dynamics of scattering and fragmentation processes in unprecedented detail. Even information on electronic energy levels of the highly charged projectiles becomes accessible with a resolution that is on a par with state-of-the-art spectroscopic methods.

In the following, selected examples of planned experiments in CRYRING are briefly discussed.

Precision spectroscopy on highly charged ions Precision spectroscopy on highly charged ions is a very active research field at GSI (see also Section 2.1). In earlier in-ring experiments investigating e.g. the $1 s$-Lambshift on highly charged hydrogen-like ions, germanium detectors were used to measure $\mathrm{x}$-ray energies emitted in Lyman transitions and a overall accuracy of about a few $\mathrm{eV}$ has been achieved (e.g. Gumberidze et al., 2005). However, this precision is not sufficient to test higher-order QED effects. Therefore, there are presently few novel x-ray detection schemes in development, based on e.g. crystal spectrometers (Beyer, 1997; Beyer et al., 2015) or micro-calorimeters (e.g. Egelhof et al., 1996; Bleile et al., 2002; Pies et al., 2012; Hengstler et al., 2015; Kraft-Bermuth et al., 2012), that provide substantially higher resolutions than semiconductor detectors. Also momentum imaging has proven already to be suitable for gaining information on the structure of highly charge ions (Fischer et al., 2002, see Figure 2.20). Here, the development of a complementary and completely novel experimental approach is proposed also exploiting kinematic information of the collision in order to obtain high-precision spectroscopic data on highly charged projectiles.

The technique discussed here is based on a similar principle as the conventional electron energy loss spectroscopy (Hillier and Baker, 1944). However, in the scheme to be realized in CRYRING the electrons are not free but loosely bound in an excited lithium atom. Furthermore, the electrons are essentially at rest in the laboratory frame but the 'sample', i.e. the highly charged ion, moves with high velocity, a situation often dubbed as 'inverse kinematics' (see also Section 2.2.2). Seen in the rest frame of the highly charged ion, a quasi-free fast electron collides with the highly charged (but not bare) ion 
in an inelastic collision, thereby exciting or even ionizing the ion. At threshold the electron loses all its energy and appears as a 'cusp electron' in the projectile continuum which can be detected with high resolution (meV in the projectile frame) by a forward magnetic imaging spectrometer (Nofal et al., 2007; Hillenbrand et al., 2014a,b, 2015) or in the MOTReMi itself. Additionally, obtaining high resolution spectroscopic data about the highly charged ion requires the measurement of the momentum change of the recoiling target ion which directly contributes to the total energy balance. Depending on the collision velocity and the observed reaction the achievable resolution can be by a factor of 2-10 higher than with conventional germanium detectors.

This kinematical method will allow obtaining precision spectroscopy data between any ground-state and excited levels of any ion species that can be stored in the ring with an accuracy comparable or even superior to all other optical (cf. Section 2.1.1) or cooler dielectronic recombination methods (cf. Section 2.1.3). It will eventually be limited only by the beam velocity uncertainty. In principle, this is the final limit for most spectroscopic methods applicable in storage rings and is, thus, similar for all methods applied. In the CRYRING this new method shall be tested and pioneered for hydrogen-like ions with nuclear charges up to 23 (vanadium).

Higher order effects in target single ionization At not too large projectile charges and at large collision velocities, the interaction between the projectile and the target is expected to be well described by perturbative first order methods, which is considered to be an 'easy' case for a theoretical treatment. However, in the fully differential cross sections for single ionization of helium colliding with $100 \mathrm{MeV} / \mathrm{u}$ $\mathrm{C}^{6+}$ ions surprising discrepancies between experimental results and state-of-the-art calculations were found (Schulz et al., 2003). For an explanation, a higher order ionization mechanism, involving the additional interaction between the projectile and the target nucleus, was proposed. A simple model neglecting interferences between the two scattering channels enabled to reproduce the experimental results (Schulz et al., 2007). Later on it was found that the interferences might be suppressed due to the small projectile coherence length, which in this experiment was orders of magnitude smaller than the target atom size (Egodapitiya et al., 2011; Wang et al., 2012). At increased projectile charge states and lower velocities, i.e. if the perturbation of the target system by the projectile gets stronger, higher order effects contribute strongly to the collision dynamics. Even though the electron emission characteristic is fairly well reproduced by theory (Moshammer et al., 1999, e.g.), for describing the fully differential cross sections essentially all available theoretical models fail completely (McGovern et al., 2010; Fischer et al., 2003b).

Apart from the correct description of the coherence properties of the incoming projectile wave and of the interaction between the projectile and individual target particles, there are two additional factors to be considered, that complicate the situation: First, the target structure and the correlation between the two electrons which are bound in the target influence the collision dynamics (Fiol and Olson, 2004; Fischer et al., 2003b). The electronic correlation in the ground state of the helium target, however, cannot be fully incorporated in a dynamical calculation and, in most of them, is not even included explicitly. Second, the experimental resolution might strongly influence the structure of the measured cross sections and, hence, may result in an impairment of the significance of the experimental results.

Due to the extremely low target temperatures achievable in magneto-optical traps the impact of the experimental resolution will be decisively reduced as compared to all earlier experiments. Moreover, due to the simple $\mathrm{H}$-like electronic structure of lithium and the helium-like structure of calcium with 
only one and two valence electrons, respectively, effects of electronic correlation can be studied in great detail. Hence, the proposed experiment in the CRYRING will provide an ultimate test for the theoretical description of the projectile-target interaction including possible multiple scattering contributions.

Correlation in target multiple ionization The investigation of multiple electron transitions in atomic targets provides a fundamental test of dynamical electronic correlation (e.g. McGuire, 1997). Previously, the helium atom was the prototype system of choice to study electronic correlations in collisions (e.g. McGuire, 1982; Schulz et al., 2001; Fischer et al., 2003a, note: there is also a vast amount of literature on electron impact, single photon, and strong laser-field induced double ionization of $\mathrm{He}$ ) mainly for two reasons: On the experimental side, it can easily be produced as an atomic target for collision experiments by means of supersonic gas-jets and, on the theoretical side, due to its rather simple structure with only two electrons its description is still manageable.

In experiments at CRYRING@ESR an attempt to study multiple ionization of lithium and calcium can be undertaken. As a three-electron system, lithium represents the next step in complexity after helium. Its loosely bound $2 s$ electron and deeply bound closed inner shell makes it a qualitatively completely different target as compared to the noble gases, which have so far been used in most experiments. From the theoretical side, lithium has attracted considerable attention in the last few years. There are numerous approaches to address the problem of triple electron emission (e.g. Emmanouilidou et al., 2008; Colgan and Pindzola, 2012). Lithium is therefore destined, to become a benchmark atomic system of the future, as helium has been in the past (Colgan et al., 2001). Calcium, in turn, represents a manyelectron system and is in this respect complementary to lithium. Here up to 20 electrons can be emitted simultaneously which allows studying few-body dynamics by a statistical analysis of the many electron continua. Up to now, not a single coincidence experiment on multiple ionization of $\mathrm{Li}$ or $\mathrm{Ca}$ has been reported.

\subsubsection{Two-center interference in charge transfer at $\mathrm{MeV}$ ion-molecule collisions}

When CRYRING was operational at Stockholm University, a significant fraction of the experimental programme involving the internal gas-jet target and its recoil-ion-momentum spectrometer was dedicated to the investigation of the dependence on the relative orientation of the incoming projectile direction and the target molecular axis in $\mathrm{MeV}$ ion-molecule charge transfer collisions. The orientation information can be extracted experimentally for charge-transfer processes that are accompanied by rapid target dissociation so that the momentum vector of one or more charged target fragments can be measured and thereby reveals the molecular orientation at the instant of the collision. For the fundamental system $\mathrm{p}+\mathrm{H}_{2}$ a very strong variation (of about a factor of two for $1.3-\mathrm{MeV}$ protons) was found in the cross section for the transfer and excitation process as a function of the molecular axis orientation (Støchkel et al., 2005). This was found to be caused by the interference of the two components of the outgoing de Broglie wave of the neutralized projectile corresponding to the electron transfer taking place in the vicinity of either of the two target nuclei (Schmidt et al., 2008). Furthermore, an even stronger orientation effect was found when double-electron capture in $\mathrm{He}^{2+}+\mathrm{H}_{2}$ collisions was considered (Misra et al., 2009).

The final experimental project with the CRYRING internal target in Stockholm concerned the transfer of core electrons from $\mathrm{N}_{2}$ to proton projectiles. Here another aspect of possible relevance to the interference behaviour should become important, namely the symmetry of the initial molecular state of the active electron. In $\mathrm{H}_{2}$ both electrons occupy single-electron states of gerade symmetry, whereas the 
core electrons of $\mathrm{N}_{2}$, depending of the preferred choice of basis, will have to be described either as localized to either nucleus or as two electrons of gerade and two of ungerade symmetry. As the expected interference pattern was believed to be inverted for capture of electrons from initial ungerade states the a priori expectation would be that no interference effects should be observed for the transfer of core electrons from $\mathrm{N}_{2}$ to a fast proton. Nevertheless, our first such investigation using 1.04-MeV protons as projectiles indicated an orientation dependence in accordance with what should be expected from the interference model presented by Schmidt et al. (2008) for electron capture from $\mathrm{H}_{2}$ without taking the initial state symmetry into account (Gudmundsson et al., 2011).

With the possible renewed opportunity to study fast ion-molecule collisions with the CRYRING internal gas-jet target at GSI, it would be very interesting to return to this problem for further investigations. One possibility would be to simply repeat the $\mathrm{p}+\mathrm{N}_{2}$ experiment at a higher projectile energy. While the measured cross section at $1.04 \mathrm{MeV}$ varies monotonically with the molecular orientation with maximum for the molecular axis transverse to the beam and a cross section reduced by $30 \%$ for parallel orientation a more dramatic and characteristic orientation dependence is foreseen by the interference model if 2-MeV projectiles are used. Here, a strong variation with angle is to be expected and an intermediate minimum in the cross section is foreseen in the interference picture for an angle between the beam and the molecular axis of about $58^{\circ}$. Hopefully these investigations will show whether the interference model is still relevant for other systems than $\mathrm{H}_{2}$ and one can move on to other targets as $\mathrm{O}_{2}$ or to break the symmetry of the initial state $\mathrm{CO}$ or NO.

Another aspect of the single measurement with molecular orientation information performed on $\mathrm{p}+\mathrm{N}_{2}$ so far, which would also call for further investigations of this collision system, is that we found an increased yield of multiply charged atomic nitrogen fragment ions for molecules oriented along the beam direction. Our interpretation of this finding is that for this orientation there is an increased probability to form target states with two core holes (one at each site) which then leads to the higher atomic charge states through multiple Auger processes. Such two-site double-core hole states are presently being investigated in some of the first x-ray free-electron laser experiments (Berrah et al., 2011) and further measurements with the internal gas-jet target may be helpful in shedding light on this related interesting field of research.

\subsubsection{Collision of highly-charged ions with a hot, dense plasma}

Research on warm dense matter (WDM) having near-solid density and low temperature of $0.1 \sim 10 \mathrm{eV}$, which is relevant to astrophysical phenomena, has been extensively pursued (Koenig et al., 2005) and a comprehensive understanding of this state of matter is a challenging issue. In order to investigate the physical property and atomic physics in WDM, various methods, such as reflected/transmitted light measurement by using an ultrashort IR laser pulse, emission spectroscopy, x-ray Thomson scattering and extended x-ray absorption fine-structure (EXAFS), have been employed so far. Relativistic beams of HCI also provide a useful tool to probe WDM produced in the laboratory, since the penetration depth is relatively long compared with the characteristic scale length of WDM. In particular, the well-defined beam in the CRYRING and the use of droplet targets will allow for a novel approach towards the investigation of the interaction of $\mathrm{HCI}$ with WDM.

A well-defined high energy-density target can be provided by heating a droplet with an ultra-high intensity laser pulse. A proof-of-principle experiment for the creation of WDM has already been per- 


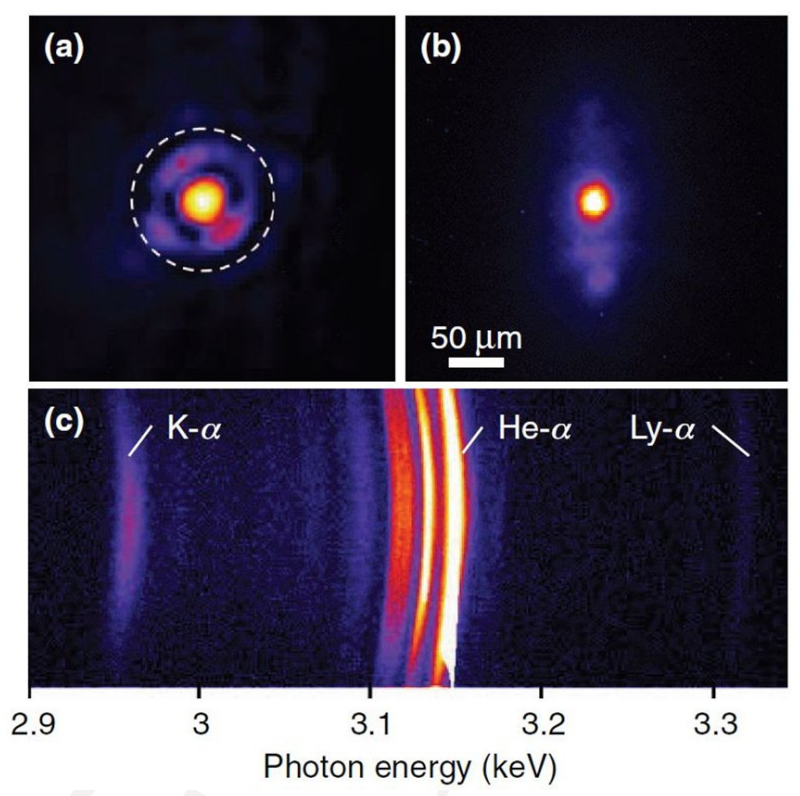

Figure 2.21. (a) Focal laser intensity distribution. The dashed circle indicates the $21 \mu \mathrm{m}$ diameter argon droplet.

(b) Time-integrated XUV image of the droplet's thermal emission. The laser pulse comes from the left.

(c) X-ray emission spectrum showing the strong argon $\mathrm{K}-\alpha$ and $\mathrm{He}-\alpha$ transition lines. The K-shell transitions in intermediate charge state argon ions are visible as satellites between the $\mathrm{K}-\alpha$ and $\mathrm{He} \alpha$ line

formed at the Petawatt high-energy laser for heavy-ion experiments (PHELIX) facility (Bagnoud et al., 2010), in which an argon droplet with a diameter of $21 \mu \mathrm{m}$ was used as a target (Costa Fraga et al., 2012). Laser pulses of $370 \mathrm{fs}$ duration, containing $2.5 \mathrm{~J}$ energy from the pre-amplifier stage, with a repetition rate of about one shot every 3 minutes, were focused onto the droplets by a $90^{\circ}$ off-axis parabola, producing a spot of $3 \mu \mathrm{m}$ in diameter (see Figure 2.21(a)) and peak intensities of around $10^{19} \mathrm{~W} / \mathrm{cm}^{2}$. Temporal synchronization was obtained by triggering the laser amplifier chain with the high-frequency signal that drives the Rayleigh breakup process of the droplet target. For the ring experiments this has to be also synchronized with the ion bunches.

Figure 2.21(b) shows a time-integrated 2D image of the XUV emission in a narrow spectral range around $13.7 \mathrm{~nm}$, evidencing a spherically shaped plasma that remains close to the initial droplet size during the thermal emission stage. This supports the conclusion that the droplets are heated isochorically to high-density plasma conditions. The droplet x-ray emission is spectrally dispersed by a cylindrically curved graphite crystal and is then recorded by a back-illuminated charge-coupled device (CCD) camera. The measured x-ray spectrum is shown in Figure 2.21(c), which covers the energy range from the cold argon $\mathrm{K}-\alpha$ line at $2.96 \mathrm{keV}$ to the $\mathrm{Ly}-\alpha$ transition at $3.32 \mathrm{keV}$. The $\mathrm{K}-\alpha$ line represents the fluorescence following collisional ionization of K-shell electrons by the relativistic electron population produced during the interaction with the intense laser pulse. The estimated conversion efficiency of the laser energy into $\mathrm{K}-\alpha$ radiation of about $10^{-5}$ indicates an efficient coupling to the relativistic electrons. The plasma condition will be maintained for a time window of roughly $1 \mathrm{~ns}$, limited by the duration of heating after the laser pulse by the refluxing electrons, and the expansion speed of the droplet. According to calculations, temperatures in the order of $100 \mathrm{eV}$ will be reached using the pump laser as foreseen for $\mathrm{x}$-ray laser experiments of the ESR, providing up to $10 \mathrm{~J}$ in $500 \mathrm{fs}$ pulses.

Here, it should be noted that a high energy-density plasma itself is a promising subject for the understanding of the interior of a giant gas planet. In fact, although relatively low temperature $(\sim 6000 \mathrm{~K})$ and ultra-high pressure $(\sim 200 \mathrm{GPa})$ condition is predicted for the deep core of Jupiter Saumon and Guillot (2004), the equation of state (EOS) of the transition phase from molecule to metallic hydrogen remains poorly understood.

In combination with the high-quality ion beams in a storage ring, such a WDM target will also allow 
for studies of processes relevant for astrophysical phenomena. For example, since completely stripped uranium ion has an extremely strong Coulomb field, many electrons bound in the bulk matter (atom) undergo the charge transfer into the $\mathrm{HCI}$, that is, $A^{q+}+B \rightarrow A^{(q-1)+}+B^{+}+\Delta E$ ( $\Delta E$ : inelastic energy gain). Once the captured electrons decay into the ground state due to subsequent radiative transition, they can never escape from the deep Coulomb potential of the ions. The interaction of such HCI with the electrons in the WDM, therefore, might be considered as a collision of an electrostatic 'black hole' with the giant gas planet.

In the CRYRING experiment, highly charged ions $\left(\mathrm{U}^{92+}\right)$ with an energy of more than $10 \mathrm{MeV} / \mathrm{u}$ (Table 1.1) will collide with the WDM target (hydrogen and argon) generated by the intense laser pulse, as described above. Then, we measure the cross section of the charge exchange and the energy change $\Delta E$ of the projectile ion $\left(A^{(q-1)+}\right)$, which provides important information on collective effects in WDM. For example, the influence by the lowering of the ionization potential, distortion of the lower electronic energy structure and band structure in the extreme condition can be studied. Moreover, excitation processes of the WDM by HCIs can be explored by x-ray spectroscopy.

\subsubsection{Intense laser-induced recombination: The inverse above-threshold ioniza- tion process}

Laser induced recombination has been extensively studied at TSR, ESR, and CRYRING (Schramm et al., 1991; Borneis et al., 1994; Asp et al., 1996). The situations at TSR (Lestinsky et al., 2008) and CRYRING (Danared et al., 1994) are peculiar, since the quality of the electron-cooler is by far exceeding the parameters reached at the other installations. Due to the extremely low energy spread of the electrons, high-resolution spectra can be expected. In addition the density of electrons in a given band above the bound states is accordingly higher. In this situation, a completely new realm can be reached by utilizing ultra-high intensity lasers to induce the recombination. In this case the inverse above-threshold ionization (IATI) phenomenon will be observable. In the IATI process, the electrons recombine with ions in an intense laser field, and high-order harmonics light corresponding to the incident laser photon energy $(h v)$ is emitted, that is, $A^{q+}+e^{-} \rightarrow A^{(q-1)+}+n h v$. Note that both even and odd harmonics are generated, which is different from the interaction between intense laser pulse and gaseous media. The IATI process has recently been studied theoretically by $\mathrm{Hu}$ and Collins (2004) who revealed that the recombination probability peaks at specific energies of the injected electron. Furthermore, it has been found that recombination into excited states due to the IATI process also occurs with high probability. Figure 2.22 shows the spectrum for the case of the recombination of a proton beam in an intense laser field $(\lambda=800 \mathrm{~nm}$, intensity: $2 \times 10^{14} \mathrm{~W} / \mathrm{cm}^{2}$, pulse width: $50 \mathrm{fs}$, initial electron energy: $\sim 3.4 \mathrm{eV}$ ), which is calculated by solving the time-dependent Schrödinger equation. The high order harmonics up to a cutoff energy at $\sim 60 \mathrm{eV}$ due to the IATI process are clearly seen.

In the experimental scheme, these studies will follow the well-established set up supported by the CRYRING lattice (see Figure 2.23). The charge exchanged $A^{(q-1)+}$ ions, and the emitted harmonics radiation in the direction of the laser will be recorded in coincidence. The novelty of these measurements lies in the fact, that the spectral shape can reveal details of the dynamics of the progress. Of practical interest is also the fact, that due to the ultra-high intensity, much higher recombination rates are to be expected, which might even be useful for non-Liouville extraction of portions of a bunch. 
Figure 2.22. The radiation spectrum for the recombination process (Hu and Collins, 2004).
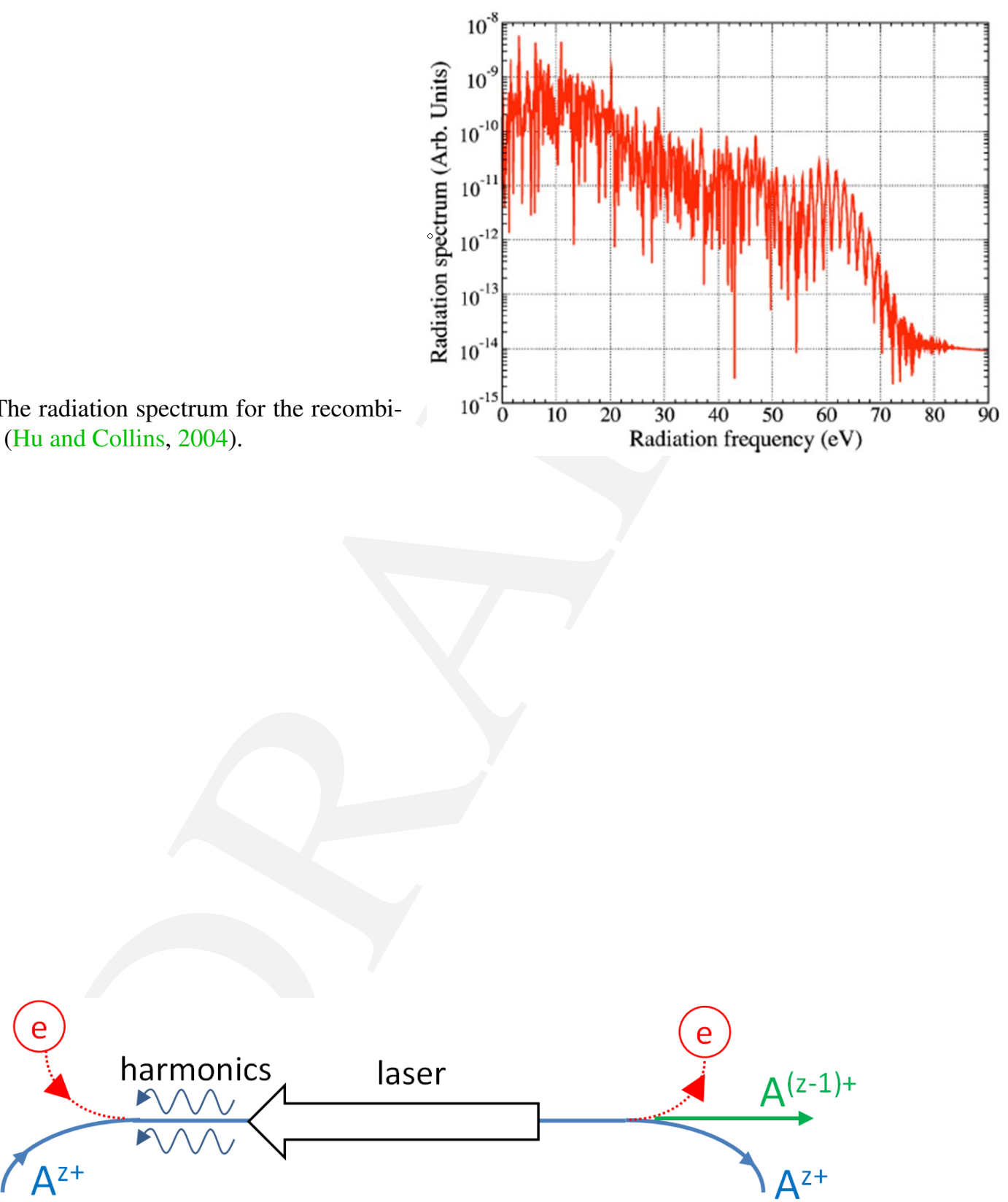

Figure 2.23. Scheme for the experimental arrangement in an IATI experiment. 


\section{Chapter 3}

\section{At the borderline of atomic and nuclear physics}

\$Id: border_physics.tex,v 1.9 2016/04/08 12:23:09 lestinsk Exp \$

\subsection{Accessing nuclear properties of stable ions and artificially in- flight synthesized radioisotopes employing electron-ion collision spectroscopy}

A relatively new application of resonant electron-ion recombination is the determination of nuclear properties from DR spectra (Brandau et al., 2015). Early measurements at CRYRING and TSR that addressed isotope effects in DR used Cu-like and Zn-like configurations (Schuch et al., 2005; Schippers et al., 2005). This choice was dictated by the limited performance of the injectors available at that time. In this respect, GSI is the ideal experimental environment for isotope-shift studies using DR. GSI provides a free choice of charge states, and even the heaviest long-lived natural element, uranium, is available with the spectroscopically preferred three-electron configuration, that, in most cases ideally combines +experimental precision with simplicity of electronic configuration (Brandau et al., 2008; Kozhedub et al., 2008; Lestinsky et al., 2008; Brandau and Kozhuharov, 2012; Bernhardt et al., 2015a).

In contrast to ions with a more complex electron shell, as typically used in isotope shift studies employing laser spectroscopy, the extraction of the nuclear data from atomic spectra of Li-like ions can be carried out at a full QED level (Brandau et al., 2008; Lestinsky et al., 2008; Kozhedub et al., 2008; Zubova et al., 2014). It is not masked by many-body effects and, thus, free of the associated ambiguity. As an example Figure 3.1 provides results of a DR isotope-shift study for the two Li-like Nd isotopes with atomic mass numbers $\mathrm{A}=142$ and $\mathrm{A}=150$. Notably, in this experiment energy shifts $\delta E^{142,150} \approx 40$ $\mathrm{meV}$ of resonances associated with excitations of several hundred $\mathrm{eV}\left[E\left(2 s-2 p_{1 / 2}\right)=139.25 \mathrm{eV}\right.$ and $E\left(2 s-2 p_{3 / 2}\right)=729.12 \mathrm{eV}$ ] were derived with uncertainties of less than $1 \mathrm{meV}$ and $3 \mathrm{meV}$, respectively (Brandau et al., 2008; Kozhedub et al., 2008). In the meantime pioneering experiments with artificially synthesized isotopes of three-electron uranium and protactinium ions could be performed (Brandau et al., 2009, 2010, 2013; Steck et al., 2010) (Figure 3.2) as well as experiments with nuclear isomers (Brandau et al., 2013) illustrating the potential of the technique.

Nuclear volume shifts and hyperfine splittings scale with large powers of the nuclear charge, i.e., 
Figure 3.1. Nuclear volume shift in the DR of the two Li-like isotopes ${ }^{A} \mathrm{Nd}^{57+}$ with mass numbers $\mathrm{A}=142$ (red) and $\mathrm{A}=150$ (black) in the energy range of the ${ }^{A} \mathrm{Nd}^{56+}\left(1 s^{2} 2 p_{1 / 2} 18 l_{j^{\prime}}\right)$ resonances (Brandau et al., 2008). The measurements were performed at the present ESR electron cooler.
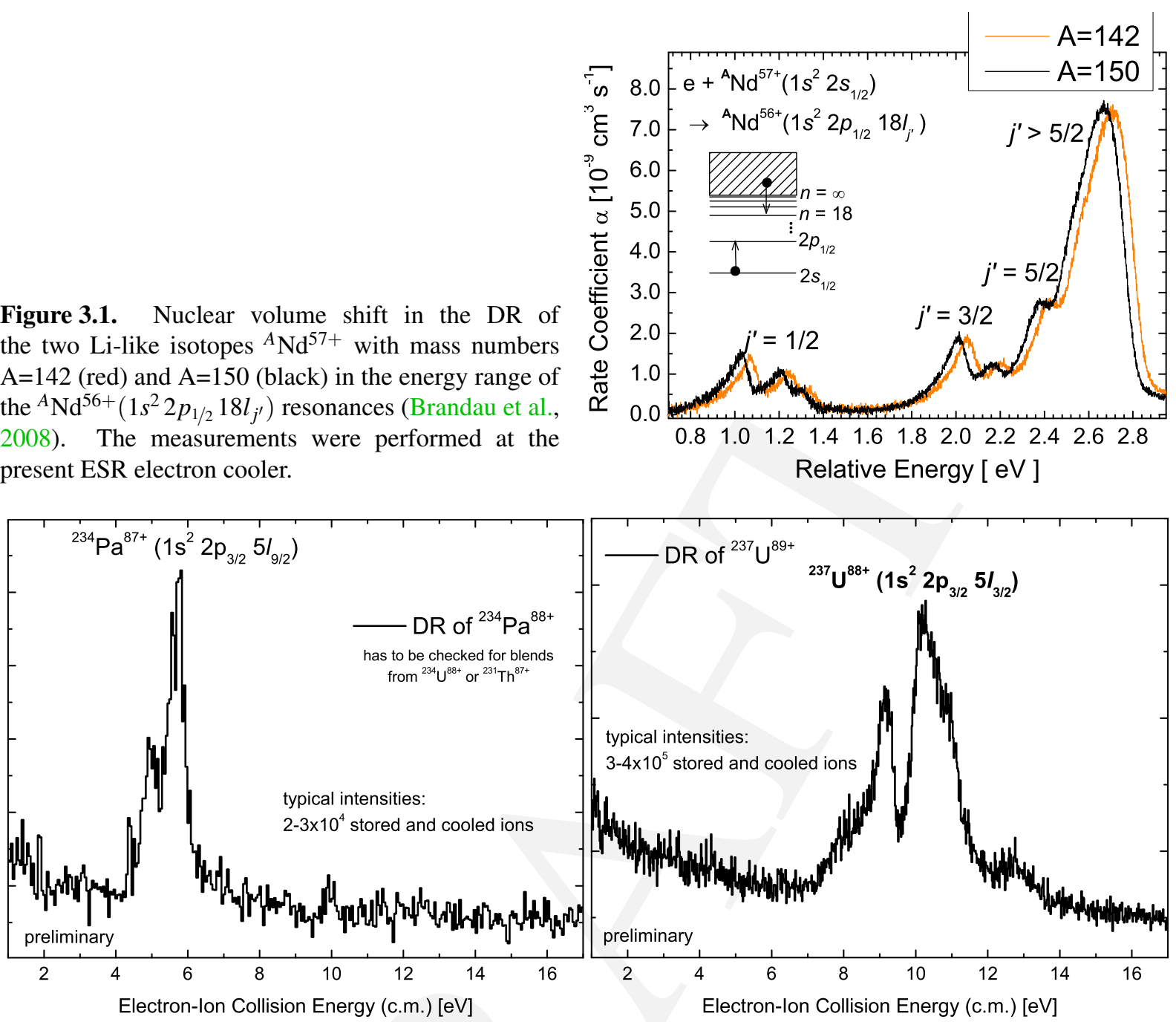

Figure 3.2. DR of the two radioisotopes ${ }^{234} \mathrm{~Pa}^{88+}$ (left) and ${ }^{237} \mathrm{U}^{89+}$ (right). The ${ }^{234} \mathrm{~Pa}$ - spectrum was obtained with intensities as low as $2-3 \times 10^{4}$ stored ions (Brandau et al., 2009, 2010, 2013; Steck et al., 2010).

with $Z^{5 \ldots 6}$ and $Z^{4}$, respectively. Consequently, the DR approach is especially well-suited for the heaviest elements. It is the ideal tool for precision studies with the heaviest highly charged ions uniquely available at the GSI facility. By using the CRYRING@ESR combination and exploiting the high resolution of the CRYRING electron cooler (Figure 2.6) a substantial boost in both, sensitivity and precision, can be expected. This is strikingly evident already from the simulations (Figure 2.6). Important topics that can be addressed using CRYRING at GSI comprise:

- High-resolution high-precision isotopic or/and isotonic energy shifts and hyperfine-splitting studies with few-electron heavy ions. Nuclear charge radii, magnetic moments and nuclear spins of stable isotopes, of radioisotopes with a lifetime $>10 \mathrm{~s}$ and even of nuclear isomers can be derived from the DR spectra. In this context, it is appropriate to highlight once more the uniqueness of such experiments due to:

- the dedicated preparation and control of the ions in ESR (Brandau et al., 2009, 2010, 2013; Steck et al., 2010),

- the possibility of the full QED treatment (three-electron ions) in the analysis of the candidates 
of interest (Lestinsky et al., 2008; Brandau et al., 2008; Kozhedub et al., 2008; Zubova et al., 2014; Bernhardt et al., 2015a).

- The sharp resonance features of DR are ideally suited for monitoring the relative intensity of associated beam admixtures. Such monitoring can be applied, for example, to cocktail beams of different isobars, atomic metastable states, hyperfine-excited states or nuclear isomers. The unique signatures of DR resonances can be exploited for:

- Lifetime measurements of the beam components, i.e., of the long-lived atomic and nuclear states but also of otherwise unresolved isobars.

- Detection and identification of reaction products, e.g., in support of laser-spectroscopy experiments.

- Enrichment, or separation of isobars, isomers or atomic metastable ions (based on the chargechanging nature of DR).

With the argument already addressed above, that the total resonance strength is conserved while the peak cross section is inversely proportional to the experimental energy spread, the contrast between the signal from the state under study and the 'background' arising from other processes with different beam components will be enhanced by up to two orders of magnitude at the CRYRING cooler (Figure 2.5).

- A central topic already in the present DR program at the ESR electron cooler is the investigation of the many facets of the lowest known isomeric states, e.g. in ${ }^{229 m} \mathrm{Th}\left(E^{*}=7.6 \mathrm{eV}, T_{1 / 2}=79 \mathrm{~h}\right.$, Beck et al. 2007), and ${ }^{235 m} \mathrm{U}\left(E^{*}=76.5 \mathrm{eV}, T_{1 / 2} 26 \mathrm{~min}\right)$. The lifetimes given here refer to neutral atoms and are distinctively different for highly charged ions due to the suppression or even absence of internal conversion. In particular, the 'nuclear clock' isomer ${ }^{229 m} \mathrm{Th}$ is in the focus of recent research worldwide due to the overwhelming opportunities that arise as soon as a handle for its dedicated investigation is found (Peik and Okhapkin, 2015; Tkalya et al., 2015; Jeet et al., 2015, and references therein). Again, the combined storage ring facility CRYRING@ESR together with the high-resolution electron target at CRYRING provide an ideal environment for such studies.

\subsection{Search for the Nuclear Excitation by Electron Capture (NEEC) process}

NEEC (Goldanskii and Namiot, 1976; Pálffy et al., 2006; Palffy, 2010) is an atomic collision process where an initially free-electron is captured resonantly into a bound atomic orbital accompanied by simultaneous excitation of the nucleus. This is the exact inverse of the decay of nuclear states by internal conversion (IC). So far, no experimental evidence has been reported for NEEC. Besides the fundamental reasons for studying the NEEC process related to the understanding of atomic-nuclear coupling, NEEC plays an essential role in astrophysical nucleosynthesis processes (Pálffy et al., 2008) and has also been discussed as a process leading to efficient isomeric triggering (Pálffy et al., 2007). Here we focus on a possible application of the planned CRYRING@ESR facility for the search for this process.

One of the often considered ways to detect the NEEC process, is to perform the nuclear excitation on a highly-excited, long-lived isomeric state. In such a case, the de-excitation of the state populated 
Figure 3.3. Known level scheme of ${ }^{129} \mathrm{Sb}$ atoms (NNDC, 2012; Genevey et al., 2003). The $\left(19 / 2^{-}\right), 17.7 \mathrm{~min}$ isomeric state decays via a $722 \mathrm{keV}$ M4 transition to the $\left(11 / 2^{+}\right)$state at $1128 \mathrm{keV}$. There is a $\left(15 / 2^{-}\right)$state which is merely $10 \mathrm{keV}$ higher than the isomer. The dominant decay of this level is via a $732 \mathrm{keV}$ transition to the same $1128 \mathrm{keV}$ level. The possible NEEC transition is indicated with a red arrow.

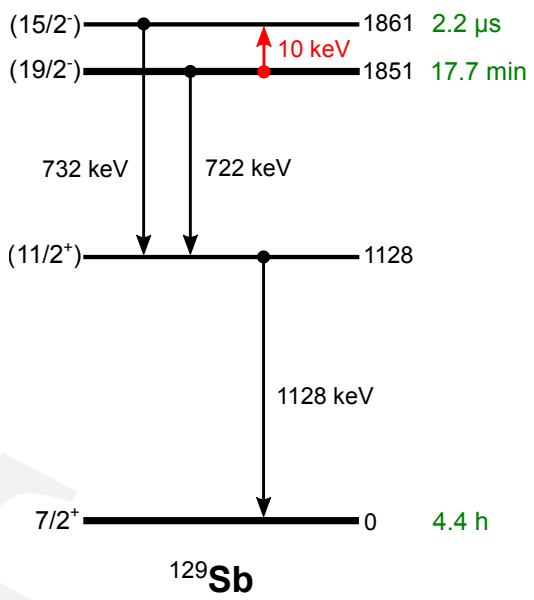

by NEEC can ideally be identified by a prompt high-energy characteristic $\gamma$-transition. During the last few years, several suitable isomeric states have been suggested. It is important to note, that the in-flight production technique employed at GSI provides very flexible conditions with access to essentially all isomeric states in all elements below uranium (Steck et al., 2010).

Here we discuss a possible experimental procedure for the example of the $17.7 \mathrm{~min}\left(19 / 2^{-}\right)$isomeric state at $1851 \mathrm{keV}$ in the neutron-rich ${ }^{129} \mathrm{Sb}$ nuclide (NNDC, 2012). We note however, that this isomer might happen to be not the best choice, but the experimental procedure will change very little if a different isomeric state is considered in a real experiment. In our chosen example, the next excited state lies at $1861 \mathrm{keV}$, that is merely $10 \mathrm{keV}$ above the isomeric state. If one succeeds to induce a NEEC transition from the isomer to this state, it can be identified by an intense $(\sim 100 \%) 732-\mathrm{keV} \gamma$-transition (see Figure 3.3).

A unique feature of the existing GSI accelerator family is that a beam of ${ }^{129 g, m} \mathrm{Sb}$ can be produced in the FRS, purified from all contaminations and then be injected into the ESR as a pure mono-isotopic beam. The high kinetic energies allow for producing ${ }^{129 g, m} \mathrm{Sb}$ as fully-ionized or few-electron ions. In addition, the ultra-high vacuum conditions allow for preserving this charge state for a long time. The ESR is a high-resolution mass spectrometer which can be used for further purification of an isomeric beam of ${ }^{129 m} \mathrm{Sb}$. The latter capability is illustrated in Figure 3.4. The Schottky spectrum reveals storage of an isobar doublet, ${ }^{140} \mathrm{Pr}^{58+}$ and ${ }^{140} \mathrm{Ce}^{58+}$ separated by a Q-value of $3388 \mathrm{keV}$. The two beam components are on different well separated trajectories which facilitates interception of one of the beam components with a mechanical scraper. Only the other component, in this case ${ }^{140} \mathrm{Ce}^{58+}$, can continue circulating the ring. In the case of ${ }^{129 m} \mathrm{Sb}$, the separation from the ground-state ${ }^{129} \mathrm{Sb}$ is slightly less than $2 \mathrm{MeV}$, which makes scraping-out the undesired component still feasible. Moreover, a better location for scraping in the ESR with a higher resolution exists and can be exploited (Scheidenberger et al., 2006).

The pure beam of ${ }^{129 m} \mathrm{Sb}$ can be slowed down in the ESR to about $4 \mathrm{MeV} / \mathrm{u}$ and transported to the CRYRING, where it can further be slowed down to several tens of keV/u. At these energies, the beam of fully-ionized or few-electron ${ }^{129 m} \mathrm{Sb}$ ions will be extracted and implanted onto a movable tape. The low kinetic energy has to be chosen to exclude Coulomb excitation of the isomer in the implantation material. The isomers will neutralize by capturing about 50 electrons. If the NEEC process occurs and populates the $1861 \mathrm{keV}$ state (indicated by the red arrow in Figure 3.3), a $732 \mathrm{keV} \gamma$-line will be emitted, which can easily be measured by a conventional $\gamma$-detector. The neutralization will be accompanied by a huge background of $<50-\mathrm{keV}$ x-rays. A suitable absorber can be applied to prevent this background radiation 

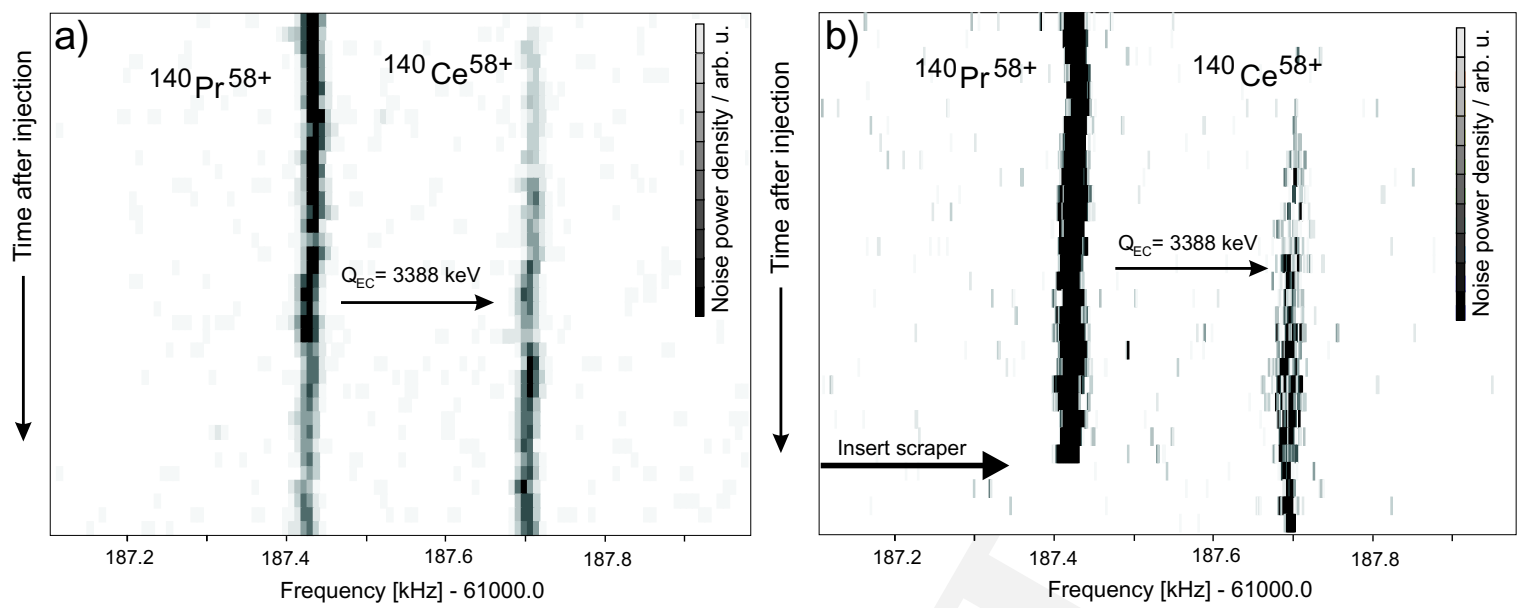

Figure 3.4. Schottky frequency spectra of a well resolved isobaric doublet separated by a Q-value of about 3.4 MeV. Pure mono-isotopic fragment beams can be separated in the FRS and injected and stored in the ESR. Left panel: undisturbed traces in time of hydrogen-like Pr and fully ionized Ce ions recorded for about $520 \mathrm{~s}$. Right panel: $170 \mathrm{~s}$ after the injection into the ESR, the beam of Pr ions was eliminated by mechanical scraping. This demonstrates the feasibility of providing pure isomeric beams in the storage ring (Scheidenberger et al., 2006; Walker et al., 2013).

from swamping the detector. The background from spontaneous decay of the isomer can be reduced by moving the tape after each implantation.

In summary, the CRYRING@ESR will facilitate unique and extremely clean experimental conditions to hunt for the yet unobserved NEEC process. The number of possible candidates for the demonstration of NEEC is huge, and since all elements can be studied at GSI the best isomeric state will be selected for the pioneering experiment. Furthermore, if the pioneering experiments are successful, a systematic investigation of the NEEC as well as nuclear excitation in electron transition (NEET) (Kishimoto et al., $2000,2006)$ processes and their dependence on the excitation-energy, transition multipolarities, atomic charge state, atomic number etc. can be envisaged.

\subsection{Laser-induced polarization of ions in storage rings}

While polarized beams of particles like electrons, protons, and muons are nowadays routinely produced and have found many applications in basic research, beams of polarized light or even heavy ions are not yet available in storage rings even though proposals have been discussed already at the end of the last century (Bosch and Egelhof, 1997). However, optical pumping of ion beams with a few keV beam energy in collinear geometry is applied (Arnold et al., 1987), e.g., at the COLLAPS setup at ISOLDE and the TRIUMF $\beta$-NMR beamline (Levy et al., 2004) in order to polarize nuclei that are afterwards implanted into crystals or other surfaces. The problem at storage rings is the conservation of polarization during the round-trip through the magnetic fields of the quadrupole and dipole magnets. It is not known whether the ion polarization will survive the fast and repetitive transition through the strong magnetic fields and gradients (Prozorov et al., 2003; Bondarevskaya et al., 2011).

The interest in polarized beams is largely motivated by the possibility of parity non-conservation effects (Bondarevskaya et al., 2011; Labzowsky et al., 2001; Zolotorev and Budker, 1997; Ferro et al., 2011). Especially in highly charged helium-like ions these effects can be very pronounced. The information on the standard model that can be extracted from such investigations is complementary to 

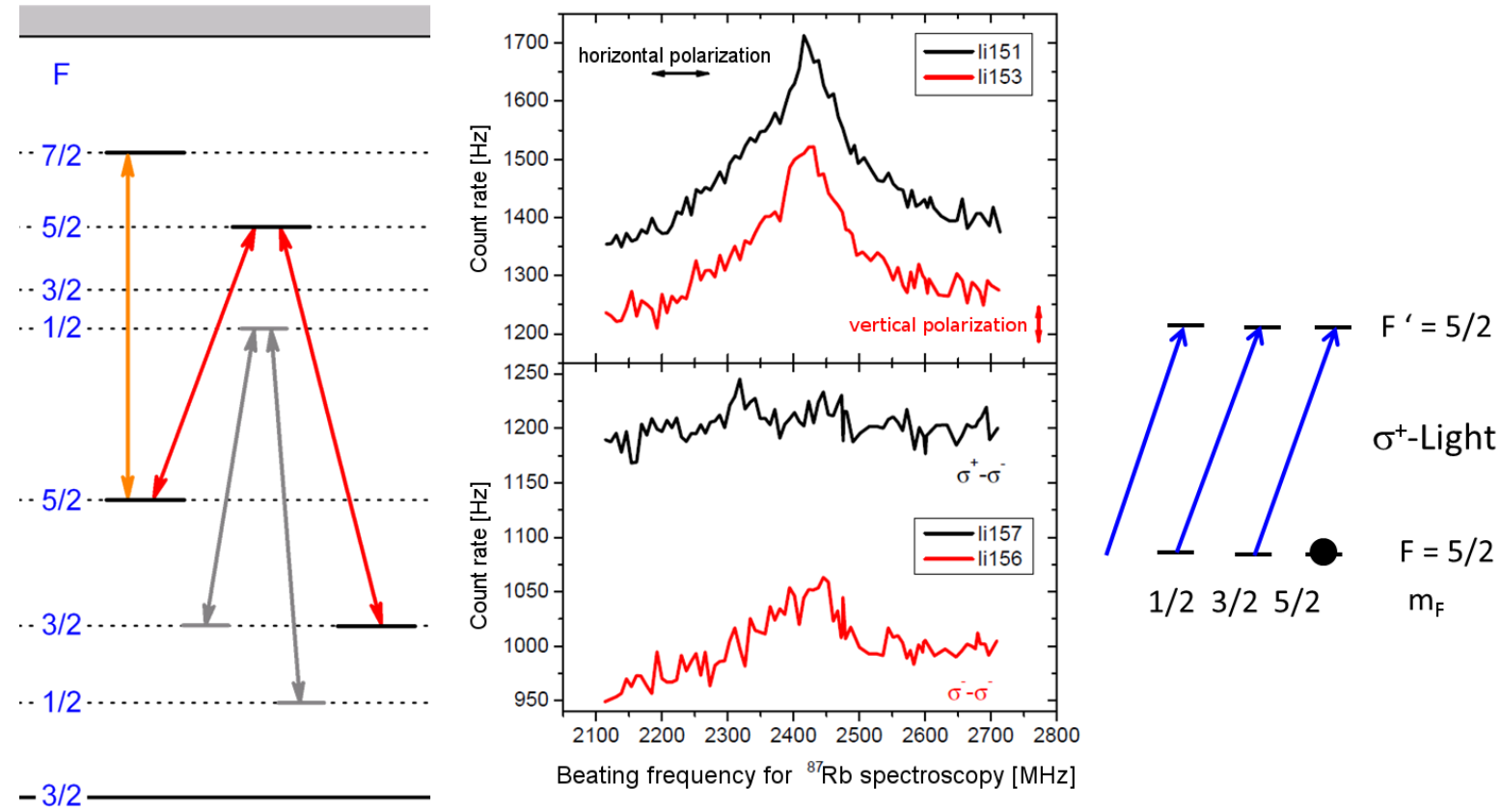

Figure 3.5. Indication for optical pumping was observed in experiments on a $\Lambda$-scheme of $\mathrm{Li}^{+}$ions (left part, red arrows) with $\beta=0.34$ at the ESR. The center frame shows the fluorescence signal observed for different polarizations. Using opposite circular polarizations leads to a disappearance of the signal as shown in the lower trace in the center. It is assumed that the optical pumping into the $m_{F}=5 / 2$ state (right) is maintained within a roundtrip of the ions in the ESR.

information obtained by the investigation of neutral systems or singly charged ions, due to the contribution of radiative corrections that are negligible in neutral atoms. For a polarization of lithium-like ions, optical pumping of hydrogen-like ions with subsequent electron capture that maintains the nuclear polarization was proposed (Bondarevskaya et al., 2011). Special consideration was given to the preservation of the obtained polarization in the storage ring experiment, since experimental information on this subject is not available.

Recently, an indication for optical polarization of an ion beam has been observed at ESR: For a test of time dilation in special relativity (SR), spectroscopy was performed in the $1 s 2 s{ }^{3} S_{1} \rightarrow 1 s 2 p{ }^{3} P_{2}$ transition in $\mathrm{Li}^{+}$ions stored in the ESR (Novotny et al., 2009). Two lasers were used to apply Dopplerreduced $\Lambda$-spectroscopy employing the $1 s 2 s^{3} S_{1} F=5 / 2 \rightarrow 1 s 2 p^{3} P_{2} F=5 / 2$ and the $1 s 2 s^{3} S_{1} F=3 / 2 \rightarrow$ $1 s 2 p{ }^{3} P_{2} F=5 / 2$ hyperfine transitions (Botermann et al., 2014) as shown in the left part of Figure 3.5. In the middle of the figure the resonance signal obtained with linear polarization is shown (top) and its disappearance as soon as one of the laser polarizations is changed to circularly polarized light. This can be understood taking into account optical pumping as depicted in the right part of the figure, where it is illustrated for the $1 s 2 s^{3} S_{1} F=3 / 2 \rightarrow 1 s 2 p^{3} P_{2} F=5 / 2$ transition. Applying $\sigma^{+}$-light, the population is transferred into the $m_{F}$ sub-state with the maximum projection along the quantization axis $m_{F}=+F$. From here, further excitation with $\sigma^{+}$-light is not possible anymore: the resonance disappears. The situation is similar for $\sigma^{-}$-light, just that the population is now transferred into the $m_{F}=-F$ state. However, this is only true, if the polarization induced by the laser, survives the revolution in the ring. To our knowledge, this would be the first time that such an optically polarized ion beam has been established in a storage ring at relativistic energies. However, we have so far only indirect signs of polarization and many more tests have to be carried out to understand the dynamics of the polarization. Moreover, due to 
the fact that the laser addresses only a small sub-population of all ions, it is not a considerable fraction of the beam that has been polarized. At maximum $1 \%$ of the ions are in the metastable ${ }^{3} S_{1}$ state and the narrow-band single-mode laser addresses only a tiny fraction of the Doppler width of about $1 \mathrm{GHz}$.

It might be that the polarization is largely produced during the excitation in front of the optical detection region. But due to the relatively long lifetime of the upper state (30 ns) and the limited interaction length, a full polarization that completely destroys the resonance signal is not likely if not at least a part of the polarization is maintained during the round trip. Since a clear conclusion about the polarization cannot be drawn from the data currently available, this topic deserves further investigation and more detailed studies. CRYRING is an ideal place for such investigations, since it is equipped with an off-line ion source and does therefore not require the full GSI accelerator facilities for such studies.

Singly charged magnesium or beryllium ions would be favorable candidates for these investigations since they provide closed two-level schemes. The most abundant magnesium isotope ${ }^{24} \mathrm{Mg}$ can be used to investigate polarization of the atomic shell, i.e. pumping in the ${ }^{2} S_{1 / 2} \rightarrow{ }^{2} P_{1 / 2}$ state with circularly polarized light should lead to dark pumping into the $m_{J}=+1 / 2$ state for $\sigma^{+}$light and $m_{J}=-1 / 2$ state for $\sigma^{-}$light. Optical polarization could clearly be proven, e.g. by applying another laser on the same transition and using $\pi$-light or the opposite circular polarization in another arm of the ring. If the signal that was pumped away previously, reappears as soon as the second laser is applied, this would manifest a clear indication for optical pumping. Additionally, a high-repetition-rate laser with e.g., picosecond pulses, adapted in the line width to the Doppler width of the electron-cooled ions could be applied to address all velocity classes simultaneously.

Once this has been successfully demonstrated, further tests including isotopes with a nuclear spin can be performed. The isotopes ${ }^{9} \mathrm{Be}$ and ${ }^{25} \mathrm{Mg}$ provide a hyperfine structure and can be used for hyperfine pumping, but this time two lasers are already required to avoid dark pumping in the hyperfine structure of the ground state. The next step would then be to address the hyperfine structure of a highly charged ion.

A particular interesting application would be optical pumping in the hyperfine transition of highly charged heavy ions, like, e.g. ${ }^{209} \mathrm{Bi}^{82+},{ }^{207} \mathrm{~Pb}^{81+}$ or ${ }^{140} \mathrm{Pr}^{58+}$. This is much more sophisticated since the slow transitions require a long durability of the produced polarization. It is of course important to measure the obtained degree of polarization. For ${ }^{140} \mathrm{Pr}^{58+}$, the electron capture decay could be an indicator of the optical polarization: It has been demonstrated at the ESR that the new, high-resolution Schottky system is capable of determining the emission direction of the electron neutrino from the recoil of the daughter ion (Litvinov et al., 2008; Kienle et al., 2013). An unpolarized beam shows the daughter ion appearing statistically with a higher or lower momentum than that expected from the electron cooler voltage. Once the beam is polarized, the daughter ion should appear always on the same side. The direction can be switched by changing the laser polarization from $\sigma^{+}$to $\sigma^{-}$or vice versa. This experiment provides information about the neutrino helicity as it was measured in the Goldhaber experiment (Goldhaber et al., 1958).

\subsection{Combining laser spectroscopy with dielectronic recombination}

Laser spectroscopy on the M1 transition in hydrogen-like (Klaft et al., 1994; Seelig et al., 1998) and lithium-like ions (Nörtershäuser et al., 2013; Sanchez et al., 2015; Nörtershäuser and Sanchez, 2015) is often hampered by the long wavelength of the transition and the very low detection efficiency for the 
emitted fluorescence photons and the strong background that is inherent in the detection of low-energy photons (Hannen et al., 2013). Partially this can be improved by the Doppler shift of relativistic ions but a more versatile technique could be based on low-background ion detection. Here, CRYRING offers the opportunity to test and develop a resonance detection using DR (Section 2.1.3 and Section 3.1). The principal idea is to look for a DR signal with the bound electron being excited in the DR process from the upper hyperfine level. No prior knowledge about the exact value of the hyperfine splitting is needed, however, in order to be able to resolve the different DR resonance structures originating from hyperfine ground and upper states the energy splitting should be larger than $50 \mathrm{meV}$ for stable ions and larger than $100 \mathrm{meV}$ for radioisotopes corresponding to transition wavelengths of approximately $24 \mu \mathrm{m}$ and $12 \mu \mathrm{m}$, respectively. These values apply for the energy resolution of the present DR set-up at the ESR. At CRYRING these limits can still be considerably lower due to the higher energy resolution that can be achieved at its cooler (compare Figure 2.5 and Figure 2.6). The higher DR resolution at CRYRING adds a further decisive benefit to the approach: in DR measurements the area of a resonance is preserved with increasing resolution, which in consequence leads to a significantly improved signal-to-background ratio of the DR-detection process and thus precision and sensitivity. Using DR as a detector for the population of the upper hyperfine state, only a small DR detection background signal will be observed as long as the laser is not in resonance, because the initial population of the upper state is negligible after a sufficiently long waiting for the initial decay of the upper hyperfine level after injection. As soon as the laser is in resonance and transfers a considerable part of the lower state population into the upper state, a DR signal will appear. Fast switching of the DR voltage between the DR energy for a signal and a second voltage in the smooth RR background region will allow for a sensitive detection. Recording the time dependence of this signal would also give access to the lifetime of the upper hyperfine state and allow us to determine even the $g$-factor of the bound electron, which also serves as a test of strong-field QED. 


\section{Chapter 4}

\section{Nuclear physics}

\$Id: nuclear_physics.tex,v 1.9 2016/04/08 12:50:09 lestinsk Exp \$

\subsection{Nuclear Structure and Nuclear Reactions}

Nuclear reactions between light charged particles are an invaluable spectroscopic probe for nuclear properties. They are employed widely in nuclear physics and are a key to understanding the evolution of nuclear matter. In storage rings, experimental conditions are exceptionally clean. Light-ion induced reactions can be performed in inverse kinematics and profit from thin windowless gas targets, repeated use of the latter due to a circulating beam and cooled beams of radioactive ions, which allow for well-defined kinematics at the target position and high energy resolution for recoil detection. The CRYRING@ESR combination provides world-unique access to such reactions in a very large energy regime without restrictions due to the chemical properties of the reaction partners. The physics case and the experimental conditions for such experiments are discussed in Egelhof et al. (2003), FAIR (2006), Grieser et al. (2012), and feasibility studies for the EXotic nuclei studied in Light ion induced reactions (EXL) project were already successfully performed at the ESR storage ring (Ilieva et al., 2007, Moeini, Ilieva, et al.; 2011; Yue et al., 2011, Zamora et al., 2015).

Recently a first reaction experiment with a stored radioactive beam was successfully performed at the ESR, where elastic and inelastic proton scattering on ${ }^{56} \mathrm{Ni}$ was investigated in inverse kinematics (von Schmid et al., 2014; Egelhof et al., 2015; von Schmid et al., 2015). For the energy range accessible at the CRYRING the investigation of one- or few-nucleon transfer reactions is a prominent example. Due to momentum matching criteria for the transferred particle(s) such investigations are most favorably performed at low incident energies of about 5-30 MeV/u.

Direct reactions studied at the internal target of CRYRING provide access to single particle neutron and proton structure in nuclei such as excitation energy spectra, angular momenta and spectroscopic factors, and matrix elements of the nucleon-nucleon effective interaction (Egelhof, 1997; Henning, 1997). These pieces of information are essential for understanding nuclear shell structure especially of neutronrich nuclei, but also for probing, e.g., the influence of the tensor component of the nucleon-nucleon interaction on nuclear structure as function of the isospin (see e.g. Schiffer et al., 2004; Kay et al., 2008). Here, an extension of experiments done with stable beams along $Z=50$ and $N=82$ becomes possible. Examples for other questions of interest are the onset of collectivity near magic numbers in the vicinity of the $N=126$ neutron shell below and above ${ }^{208} \mathrm{~Pb}$. 
The detection of light ejectiles is possible via highly segmented silicon or diamond detectors, while beam-like particles can be momentum-analyzed by the bending magnets of CRYRING, using appropriate position-sensitive detectors downstream from the magnet. Here the availability of fully ionized beams greatly reduces ambiguities due to charge states. Detection systems need to be able to operate under ultrahigh vacuum (UHV) conditions. These challenges can be met; the EXL collaboration tested successfully a number of detector prototypes, which can be employed in storage-ring experiments. Even highly segmented silicon detectors can operate in a UHV environment (Moeini, Ilieva, et al., 2011; Streicher et al., 2011; Mutterer et al., 2015). Reaction cross sections and $l$-selectivity can be optimized by choosing a suitable beam energy.

\subsection{Reaction measurements for nuclear astrophysics}

Fusion reactions of charged particles in stellar interiors are responsible for the creation of the majority of the elements lighter than iron and nickel. It is believed that about one half of the elements from iron to bismuth is produced in the so-called slow neutron capture process, s-process. Another half and all of the thorium and uranium are synthesized through the rapid-neutron capture process, $r$-process. However, these two processes can not explain the production of about 35 neutron-deficient nuclei between Se and Hg (Reifarth et al., 2014). It is presently assumed that these nuclei are the products of the photo-dissociation processes like $(\gamma, \mathrm{n}),(\gamma, \mathrm{p})$, and $(\gamma, \alpha)$ reactions on heavy $s$ - and $r$-process seed nuclei. This is the so-called $p$-process. High temperatures of about $2-3 \cdot 10^{9} \mathrm{~K}$ are required and, therefore, the favoured sites for this process are the explosively burning O/Ne layers in type II Supernovae. The needed temperatures are maintained for about $1 \mathrm{~s}$ at densities of $\approx 10^{6} \mathrm{~g} / \mathrm{cm}^{3}$ (Lambert, 1992). Photodissociation $((\gamma, \mathrm{n}),(\gamma, \mathrm{p})$ and $(\gamma, \alpha))$ and capture $((\mathrm{p}, \gamma))$ reactions involve several hundreds of nuclides, where the capture reactions are more important for lighter nuclei. An example of the reaction networks is illustrated in Figure 4.1. As the temperature decreases after the explosion, the reaction path moves back to the region of stable nuclei.

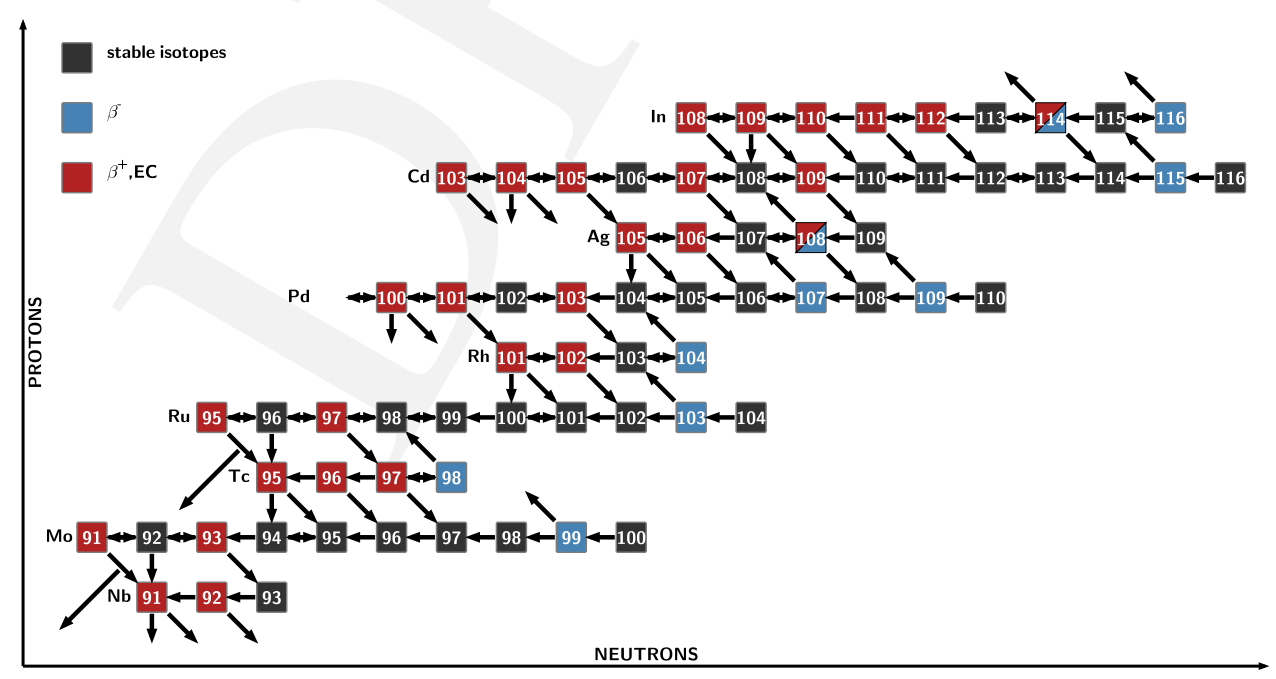

Figure 4.1. Example of the $p$-process reaction network on the chart of the nuclides. Shown is the region between niobium and indium. The assumed temperature is $\mathrm{T}_{9}=2.4$. Main reactions are the $(\gamma, \mathrm{n})$ photodissociations. Other reactions are shown, when they are dominating. The $\beta$-induced decays occur only after the explosion. See Arnould and Goriely (2003) for more details. 
The number of reactions involved in the $p$-process is huge and it will hardly possible to address them all experimentally. Therefore, the $p$-process studies will have to rely on theoretical reaction rates, obtained, e.g., with a Hauser-Feshbach statistical model. However, the calculations have to benchmarked by experimental cross sections. Uncertainties of calculated cross-sections reach several hundred percent even for stable nuclides and rapidly increase when moving away from the stable nuclei.

For the photodissociation $(\gamma, \mathrm{n})$ reactions, there is a sufficient basis of experimental data on stable nuclei, which allows for constraining theoretical calculations such that the theoretical uncertainties for stable nuclei can be reduced to a level of about 30\% (Rauscher and Thielemann, 2000). However, for the $(\gamma, \mathrm{p})$ and $(\gamma, \alpha)$ reactions, the number of measured reaction rates in the Gamow window of the $p$-process is very scarce. Due to this, the calculated rates are uncertain by factors of two to three even for the stable nuclides (Rauscher and Thielemann, 2004). As a consequence, the element abundances obtained from $p$ process models are reproduced within a factor of about three (Arnould and Goriely, 2003). No $p$-process models can explain the relatively large abundances of ${ }^{92,94} \mathrm{Mo}$ and ${ }^{96,98} \mathrm{Ru}$, the so-called Mo-Ru anomaly.

An alternative origin of these Mo-Ru nuclei could be imagined via $(\mathrm{p}, \gamma)$ reactions. One of the possibilities could be the rapid-proton capture process, $r p$-process, which powers novae or x-ray bursters (Schatz et al., 1998). In this process, the hydrogen layer on the surface of a neutron star is explosively burned providing high temperature and density conditions sufficient for the $(\mathrm{p}, \gamma)$ reactions to proceed with significant rates. However, there is presently no understanding how the produced matter could be ejected from the neutron star surface. Another possibility could be the $p$-process during type Ia Supernovae, where recent model calculations suggest the importance of $(\mathrm{p}, \gamma)$ rates for the production of light, neutron-deficient molybdenum isotopes (Howard et al., 1991; Kusakabe et al., 2011; Travaglio et al., 2011).

Photo-dissociation rates of $(\gamma, \mathrm{p})$ and $(\gamma, \alpha)$ reactions can be obtained from the proton and $\alpha$ induced $(p, \gamma)$ and $(\alpha, \gamma)$ reactions via the detailed balance principle (Glorius et al., 2014). Furthermore, direct $(p, \gamma)$ and $(\alpha, \gamma)$ capture reactions are themselves important for the $p$ - and $r p$-process network calculations (Arnould and Goriely, 2003; Schatz et al., 1998).

Addressing $(p, \gamma)$ and $(\alpha, \gamma)$ reactions in the astrophysically interesting energy range, Gamow window, is a challenge. It is difficult already for the stable nuclei. However, the majority of the nuclei involved in the $p$-process are radioactive. The investigation of these reactions is probably the best to be done in inverse kinematics where the produced radioactive beam is impinged into the hydrogen or helium target. At many facilities in the world such experiments are performed/planned with using an external target. A storage ring offers the possibility to re-use the produced exotic nuclei by storing them at a defined energy and to measure the reaction rates of interest by intersecting the beam with the internal hydrogen or helium thin, pure gas-jet target. The bending magnets of the storage ring are used as a magnetic spectrometer to analyze the reaction products, which can be detected with high efficiency. CRYRING@ESR turns to be a versatile tool for such measurements. A proof-of-principle experiment has been performed at the ESR ring of GSI, where the cross section of the proton capture on stable ${ }^{96} \mathrm{Ru}$ nuclei $\left({ }^{96} \mathrm{Ru}(\mathrm{p}, \gamma){ }^{97} \mathrm{Rh}\right)$ was measured (Zhong et al., 2010; Mei et al., 2015).

In order to perform measurements in the Gamow window of the $p$-process, the stored ions have to be slowed down to energies between $2 \mathrm{MeV} / \mathrm{u}$ and $5 \mathrm{MeV} / \mathrm{u}$. The beam life-times of such low-energy ions in CRYRING are much higher than in ESR, which makes the combination of ESR and CRYRING ideal. For many-electron ions, intersection with the gas target will lead to electron stripping, which is the main beam loss mechanism. Furthermore, due to momentum conservation, single electron stripping 


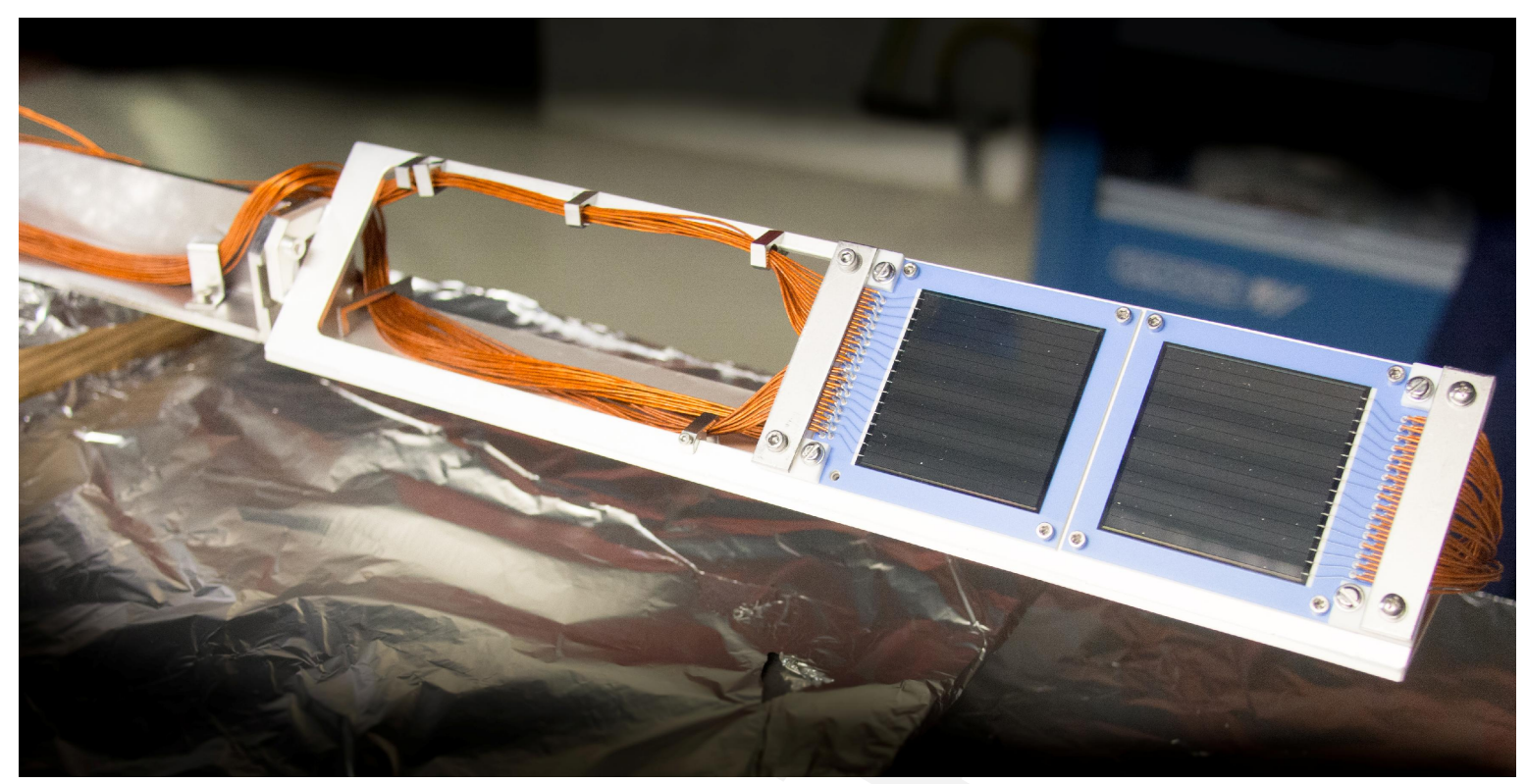

Figure 4.2. double-sided silicon strip detector (DSSD) as used to detect (p, $\gamma$ ) reaction products inside the ESR (Mei et al., 2015). The DSSD were successfully baked and used under the UHV conditions of the ESR.

and proton capture reaction products have identical magnetic rigidities after the target and thus identical positions behind the dipole magnet. Therefore, it is of a clear advantage to employ fully-stripped ions for these studies especially that they can routinely be provided at ESR. In ESR, the fully-stripped ions can be slowed down from about $100 \mathrm{MeV} / \mathrm{u}$ to an energy acceptable for CRYRING. In CRYRING itself, the ions will then be further slowed to the the required low energies of $2 \mathrm{MeV} / \mathrm{u}$ to $5 \mathrm{MeV} / \mathrm{u}$ and the actual $(\mathrm{p}, \gamma)$ and $(\alpha, \gamma)$ measurement will be performed.

The reaction products will be counted by dedicated silicon detectors, Figure 4.2 installed on the inner side of the ring behind one of the dipole magnets. Such detectors can be used under UHV conditions and have very good energy and position resolution. In addition to the position measurements, the dead layer in front of the detectors can be used to discriminate background event. It can be adjusted between about $5 \mu \mathrm{m}$ and about $100 \mu \mathrm{m}$. It is therefore possible to optimize the dead layer in order to increase the energy difference between the desired reaction products and background products. For example, if the above mentioned ${ }^{96} \mathrm{Ru}(\mathrm{p}, \gamma){ }^{97} \mathrm{Rh}$ reaction is investigated at $4 \mathrm{MeV} / \mathrm{u}$, the total kinetic energy $E_{\text {kin }}$ of the ${ }^{96} \mathrm{Ru}$ ion after the pick up of one electron is still $384 \mathrm{MeV}$. However, $E_{\text {kin }}$ of the ${ }^{97} \mathrm{Rh}$ ion after the proton capture is $380 \mathrm{MeV}$. The energy loss in a dead layer is approximately proportional to the square of the ion's charge. Hence after a dead layer of $10 \mu \mathrm{m}$ the energy of the Ru ions would still be $257 \mathrm{MeV}$ while the energy of the $\mathrm{Rh}$ ions would be reduced to $249 \mathrm{MeV}$ corresponding to a difference of $3 \%$. Such a difference can be resolved with silicon detectors.

Ions capturing an electron from the gas-jet atoms move on the outer orbits of the ring and can also be detected by a dedicated counter. The theoretical description of this atomic process is well developed 
and the corresponding uncertainty in the order of a few percent can be expected. Therefore, the capture reaction rate can be used as an in situ calibration of the luminosity.

The number of cases for possible studies is huge, in particular since not only reactions important for the $p$-process can be studied, but also $(\mathrm{p}, \gamma)$ and $(\mathrm{p}, \mathrm{n})$ reactions for the $v p$-process or fusion reactions important for the understanding of the different burning stages of the stars. It is suggested to start the experimental program with stable beams (e.g. ${ }^{86} \mathrm{Kr},{ }^{112} \mathrm{Sn}$ ). The data are hardly available and even the first experiments will make high scientific impact. The cross sections for $(\mathrm{p}, \gamma)$ and $(\alpha, \gamma)$ reactions in the Gamow window of the $p$-process are relatively high. For the first experiments one can select the cases with cross sections in the range of $0.1 \mathrm{mb}$ to $10 \mathrm{mb}$ and, thus, moderate intensities of only a few $10^{5}$ stored ions would be required.

\subsection{Direct measurements of nova $(p, \gamma)$ resonant reaction rates}

Approximately $30 \%$ of main sequence (MS) stars in our galaxy are observed to be within binary star systems (Lada, 2006). Systems containing constituents of disparate masses can evolve to a point where one star has become a white dwarf (WD), while the companion still lies on the MS. Eventually, when the MS companion becomes a red giant (RG), hydrogen-rich material from the outer envelope of the RG can cross over the Roche-lobe and be captured into the WD's gravitational well. Losing angular momentum, this material will spiral down onto the WD's surface; its kinetic energy is thereby converted into heat. Here, under high density, this material forms a hot, partially degenerate envelope surrounding the WD (José et al., 2006), with the material at the base of the envelope having highest degeneracy and density. Nuclear burning will occur, therefore, in a thin radial zone at the interface between the base of the envelope and the surface of the WD. The burning is first initiated by the proton-proton chains which, themselves, drive up the temperature in the burning zone. When the temperature reaches $\sim 50 \mathrm{MK}$, the nuclear energy generation rate becomes dominated entirely by CNO-cycle burning (Starrfield et al., 1972; José and Hernanz, 1998). At this temperature, the CNO-cycle burns with an energy generation rate $\varepsilon(T) \propto T^{13}$, where $T$ is the temperature, which is extremely sensitive to changes in temperature, and it does so while under degenerate conditions. This sets the stage for a thermonuclear runaway (TNR): the ongoing CNO-cycle nuclear burning, combined with the degenerate conditions, rapidly drives up the temperature of the burning shell without it subsequently expanding and cooling. Meanwhile, proton captures onto the seed nuclei comprising the WD surface begin to occur.

When the underlying WD is the oxygen-neon type (ONe type), proton capture processes beginning on the seed nuclei ${ }^{20} \mathrm{Ne}$ allow the throughput of the $(p, \gamma)$ reaction flow to produce a higher abundance fraction of nuclei between $20 \leq A \leq 40$ compared to the abundances produced in the case of a carbonoxygen WD. Some $\sim 100-1000$ s later, with the temperature exceeding $\sim 200 \mathrm{MK}$ (depending on the model), the degeneracy of the envelope is lifted when the temperature within the envelope exceeds the Fermi temperature of the degenerate matter, the envelope luminosity exceeds the Eddington luminosity limit, and the envelope, with freshly forged elements, is explosively ejected into space (José and Hernanz, 1998; Wanajo et al., 1999; José et al., 2001). These events are known as novae.

Within the TNR, resonant proton-capture reactions are responsible for pushing the matter flow up to $A \approx 40$. The resonant $(p, \gamma)$ reaction rate, $\langle\sigma v\rangle$, is given by a sum over contributing resonance states lying in the Gamow window of the compound nucleus. If the resonance energy, $E_{r}$, is known then a direct measurement of the resonance strength, $\omega \gamma$, completely determines the reaction rate. 
Figure 4.3. $\beta$-decay scheme for ${ }^{34} \mathrm{Cl}$ (Firestone, 1996). The ${ }^{34 m} \mathrm{Cl}$ isomeric state at $146 \mathrm{keV}$ decays into excited states of ${ }^{34} \mathrm{~S}$ producing three $\gamma$-rays of astronomical interest. $\beta$-decay branchings are given as percentages.

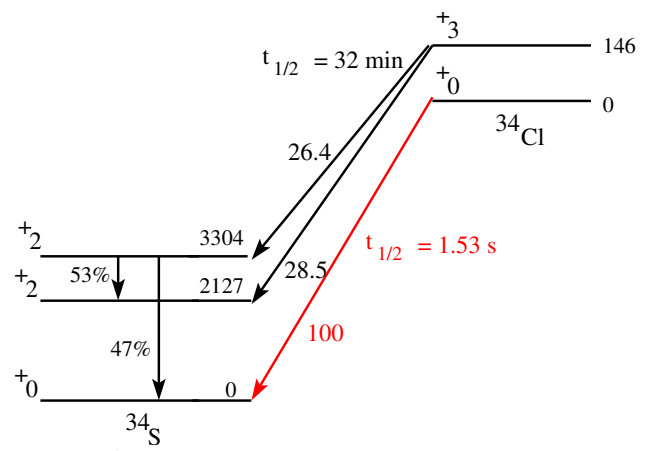

Figure 4.4. The $\mathrm{S}-\mathrm{Cl}$ burning cycle. $\beta$-decays are depicted with blue arrows and $(p, \gamma)$ reactions are depicted with vertical red arrows. The cyclic nature is depicted by the thick outer red arrow for the $(p, \alpha)$ reaction on ${ }^{35} \mathrm{Cl} .{ }^{34} \mathrm{Cl}$ is the relevant $\gamma$-ray emitter.

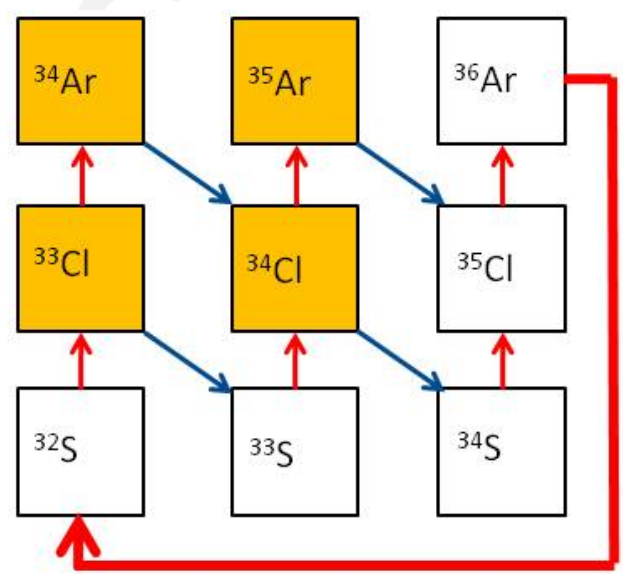

CRYRING@ESR can be used to constrain/measure the resonant $(p, \gamma)$ reaction rate for at least two reactions in the sulfur-chlorine $(\mathrm{S}-\mathrm{Cl})$ cycle of oxygen-neon novae. These reactions are intimately connected to the production of a potential astronomical $\gamma$-ray emitter, the isomeric state of ${ }^{34 m} \mathrm{Cl}$, and the expected isotopic ratios of the sulfur isotopes in presolar grains (star dust).

\section{Gamma-ray astronomy and the ${ }^{34 m} \mathrm{Cl}$ thermonuclear 'Thermometer'}

Theoretical nova models calculating the nucleosynthetic output of the each nova-type show that, within the burning zone of the ONe-type, the composition mass-flow reaches as high as calcium (José and Hernanz, 1998). Within this mass range exist three $\gamma$-ray emitting isotopes which are, themselves, potential targets of $\gamma$-ray astronomy. They are: ${ }^{22} \mathrm{Na}$ with $t_{1 / 2}=2.6 \mathrm{yr},{ }^{26} \mathrm{Al}$ with $t_{1 / 2}=7.2 \times 10^{5} \mathrm{yr}$, and ${ }^{34 m} \mathrm{Cl}$ with $t_{1 / 2}=32 \mathrm{~min}$. In the latter case, ${ }^{34 m} \mathrm{Cl} \beta$-decays into various excited states of ${ }^{34} \mathrm{~S}$ (see Figure 4.3), giving rise to the following $\gamma$-rays (and population fractions): $1.18 \mathrm{MeV}(14 \%$ ), $2.13 \mathrm{MeV}$ $(42 \%)$ and $3.30 \mathrm{MeV}(12 \%)$. Since the $\gamma$-rays originate from the decay of radioactive parent nuclei $\left({ }^{22} \mathrm{Na}\right.$, ${ }^{26} \mathrm{Al}$ and ${ }^{34 m} \mathrm{Cl}$ ) produced within the TNR, the detection of any one of these lines in nova ejecta would provide a direct isotopic abundance determination of the corresponding radioactive parent. This, in turn, would impose demanding constraints on the predictive abilities of theoretical novae models.

The S-Cl cycle is schematically illustrated in Figure 4.4. The production of ${ }^{34 m} \mathrm{Cl}$ proceeds via only one $(p, \gamma)$ path: ${ }^{32} \mathrm{~S}(p, \gamma){ }^{33} \mathrm{Cl}(\beta v){ }^{33} \mathrm{~S}(p, \gamma){ }^{34 m} \mathrm{Cl}$. The ${ }^{34 m} \mathrm{Cl}$ isomeric state is produced via feeding from the $\gamma$-ray decay of resonant states in ${ }^{34} \mathrm{Cl}$ that lie in the Gamow window of the ${ }^{33} \mathrm{Cl}(\beta v){ }^{33} \mathrm{~S}(p, \gamma){ }^{34 m} \mathrm{Cl}$ reaction. The path ${ }^{32} \mathrm{~S}(p, \gamma){ }^{33} \mathrm{Cl}(p, \gamma){ }^{34} \mathrm{Ar}(\beta v){ }^{34} \mathrm{Cl}$ entirely by-passes the ${ }^{34 m} \mathrm{Cl}$ isomeric state because the ${ }^{34} \mathrm{Ar} \beta$-decay feeds almost entirely into the ${ }^{34} \mathrm{Cl}$ ground state $(94 \%)$, while the remaining $\sim 6 \%$ of the decay feeds into excited states which, themselves, $\gamma$-decay with branches that by-pass the isomer (Endt and Firestone, 1998; Firestone, 1996). Thus, the production of the three astronomical $\gamma$-ray 
lines [1.18 MeV (14\%), $2.13 \mathrm{MeV}(42 \%)$ and $3.30 \mathrm{MeV}(12 \%)]$ arising from the $\beta$-decay of ${ }^{34 m} \mathrm{Cl}$ comes purely from $(p, \gamma)$ capture on ${ }^{33} \mathrm{~S}$. Within these reaction paths the $\beta$-decay rates of ${ }^{33} \mathrm{Cl}$ and ${ }^{34} \mathrm{Ar}$ are both known (Endt and Firestone, 1998); however, it must be stressed that both the ${ }^{33} \mathrm{Cl}(p, \gamma){ }^{34} \mathrm{Ar}$ isomericbypass reaction is entirely unknown at the present, as is the ${ }^{34 g, m} \mathrm{Cl}(p, \gamma)$ (g = ground state) destruction rate. Both are presently modelled employing the Hauser-Feshbach statistical model for their reaction cross-sections. The level densities of both ${ }^{34} \mathrm{Ar}$ and ${ }^{35} \mathrm{Ar}$, within the ONe-nova Gamow window, do not justify this treatment, as can be seen in Figure 4.5, where the vertical red bars indicate the Gamow window for the labelled temperature (in units of GK). Those states lying within the excitation energy range spanned by the 0.1 and $0.3 \mathrm{GK}$ lines can contribute to resonant $(p, \gamma)$ capture at ONe nova temperatures. Additionally, the ${ }^{33} \mathrm{~S}(p, \gamma){ }^{34 g, m} \mathrm{Cl}$ reaction rate and the subsequent model yields of ${ }^{34 m} \mathrm{Cl}$ and ${ }^{34 g} \mathrm{Cl}$ will require revision owing to work done at the Maier Leibnitz Tandem Laboratory in Munich; seven new states within the Gamow window have been found in ${ }^{34} \mathrm{Cl}$ (Parikh et al., 2009); their $(p, \gamma)$ resonance strengths have yet to be determined. Presently, their decay branchings into the ${ }^{34 m} \mathrm{Cl}$ isomeric state only have estimated upper limits, in terms of partial strengths (resonance strength times decay branching), of $\sim 1 \mathrm{meV}$ (Freeman et al., 2011).

With its relatively short half-life of $32 \mathrm{~min},{ }^{34 m} \mathrm{Cl}$ will undergo $\beta$-decay predominantly during the opaque phase of the expanding ejecta. Furthermore, it has been calculated (Coc et al., 2000) that the isomeric level can be destroyed via photo-excitation to higher levels which subsequently branch, via $\gamma$ decay, to the ${ }^{34} \mathrm{Cl}$ ground state with larger branchings than that to return back to the isomer. This effect is highly temperature dependent, with the result that the effective ${ }^{34 m} \mathrm{Cl}$ half-life is reduced to $\sim 1 \mathrm{~s}$ at temperatures around $200 \mathrm{MK}$ (Coc et al., 2000), greatly reducing the survival probability of this isotope into the ejecta phase (unless, of course, convection serves to transport a sizable fraction of it out of the peak temperature zone to the cooler surface zones of the envelope). Thus, it may well be that the $\gamma$-ray flux from this isotope may go undetected unless there is a nearby ONe nova event (Leising and Clayton, 1987). However, should it ever be detected, the subsequent ${ }^{34 m} \mathrm{Cl}$ abundance derived therefrom could help, using a backward iterative method with nova models, to place empirical constraints on the maximum TNR temperature. But this possibility can only be meaningful with improved nova models in which the aforementioned $(p, \gamma)$ reaction rates in this mass range are also improved.

\section{Sulfur isotopes in nova presolar grains}

In addition to $\gamma$-ray astronomy, presolar grains ('stardust') potentially offer a chance to constrain nova models based on measurements of isotopic ratios of key elements locked within the grains. In the last decade, a handful of presolar grains have been discovered that possess the signatures of $\mathrm{ONe}$ novae parentage (Amari et al., 2001; José et al., 2004). This conclusion is based on a comparison of their measured ${ }^{14} \mathrm{~N} /{ }^{15} \mathrm{~N}$ to ${ }^{12} \mathrm{C} /{ }^{13} \mathrm{C}$ ratios (which are related to the $\mathrm{CNO}$ cycle in the early stages of the TNR) along with the measured ${ }^{16} \mathrm{O} /{ }^{18} \mathrm{O}$ to ${ }^{16} \mathrm{O} /{ }^{17} \mathrm{O}$ ratios, with the results of ONe nova models in which the WD mass and degree of mixing were both varied (Amari et al., 2001; José et al., 2004). However, the parentage interpretation is not without problems: to achieve reasonable agreement between these measured grain ratios to the nova model predictions, a mixing factor $>95 \%$ of solar-like material from the companion star must be used (Amari et al., 2001) to effectively dilute the model ratios to match those of the grains. A theoretical model that explains this is still absent.

Sulfur abundance results, $X_{i}$, from several one-dimensional hydrodynamical ONe nova models indicate that ${ }^{32,33,34} \mathrm{~S}$ will have overproduction factors $f_{i} \equiv X_{i} / X_{\odot}$ of $\sim 100,150$ and 11 , respectively, in the 


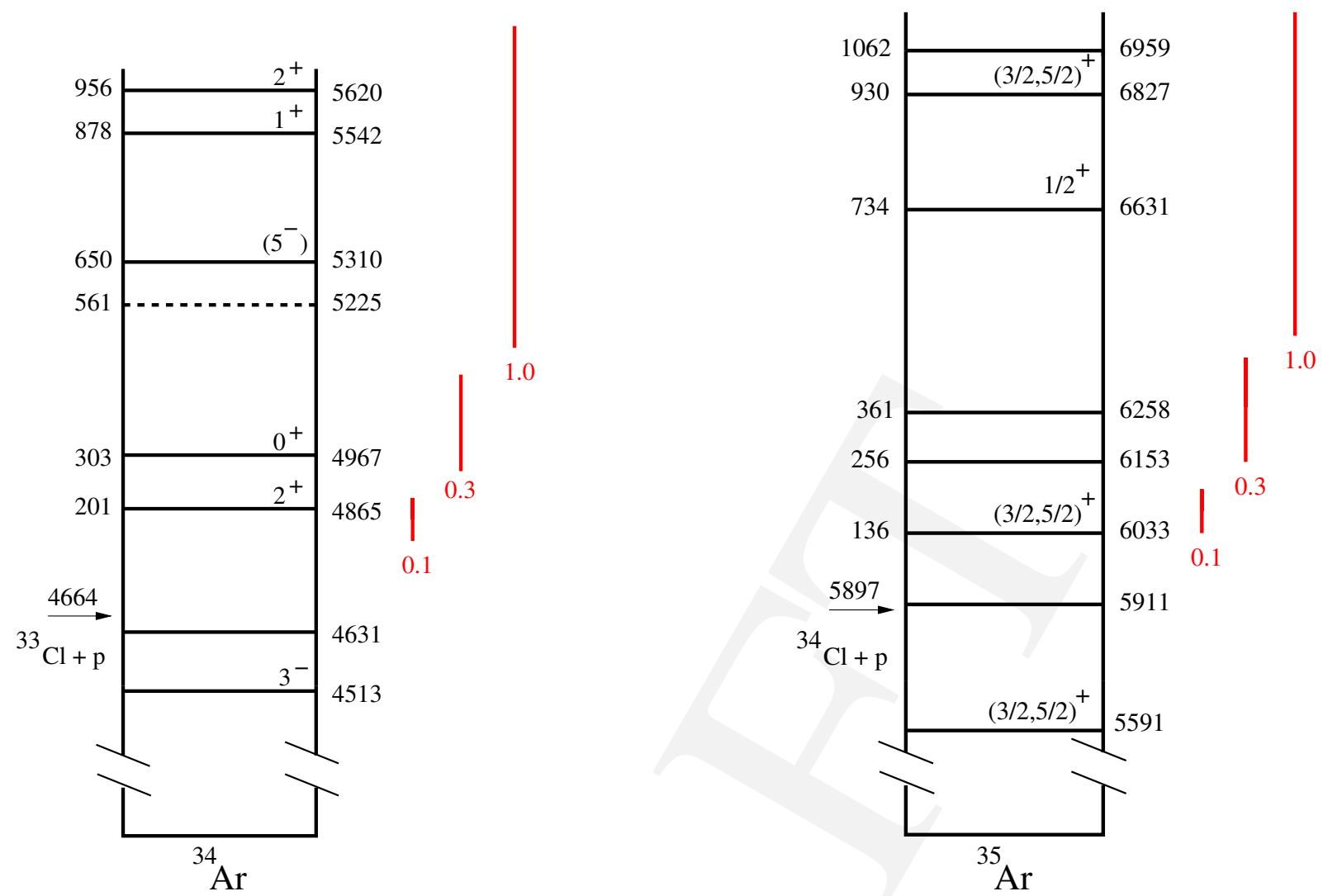

Figure 4.5. Level schemes of ${ }^{34} \mathrm{Ar}$ (Endt, 1990) and ${ }^{35} \mathrm{Ar}$ (Endt and Firestone, 1998) showing the states of astrophysical interest along with their associated excitation energy, resonance energy and spin-parity assignments. The vertical red lines indicate the Gamow windows for $(p, \gamma)$ capture with temperatures indicated in units of $10^{9} \mathrm{~K}$. The state at $E_{x}=5225 \mathrm{keV}$ in ${ }^{34} \mathrm{Ar}$ is only tentatively assigned (Grawe et al., 1974). Spinparity assignments are shown nested inside on the right, while resonance energies and excitation energies are, respectively shown on the outer left and outer right of each scheme.

ejecta (see José et al., 2001; José et al., 2004)). Here, $X_{\odot}$ is the solar abundance of each isotope. Alternatively, the model results imply isotopic ratios of ${ }^{32} \mathrm{~S} /{ }^{33} \mathrm{~S} \approx 94$ and ${ }^{33} \mathrm{~S} /{ }^{34} \mathrm{~S} \approx 2$ and ${ }^{32} \mathrm{~S} /{ }^{34} \mathrm{~S} \approx 200$. These model results, however, are again based on unmeasured reaction rates in the $\mathrm{S}-\mathrm{Cl}$ cycle. Referring back to Figure 4.4, it is clear that the production of ${ }^{33} \mathrm{~S}$ will depend on the unmeasured ${ }^{33} \mathrm{Cl}(p, \gamma){ }^{34} \mathrm{Ar}$ reaction rate, along with its own unmeasured destruction rate ${ }^{33} \mathrm{~S}(p, \gamma){ }^{34} \mathrm{Cl}$. Neither of these rates have been measured and, moreover, these sulfur abundance predictions are based on a theoretical ${ }^{33} \mathrm{~S}(p, \gamma){ }^{34} \mathrm{Cl}$ rate (José et al., 2001) computed from the then known ${ }^{34} \mathrm{Cl}$ level scheme. As mentioned above, seven new, hitherto unknown, levels above the proton threshold of ${ }^{34} \mathrm{Cl}$ and within the ONe nova Gamow window were discovered recently (Parikh et al., 2009). Upper limits for the ${ }^{33} \mathrm{~S}(p, \gamma){ }^{34} \mathrm{Cl}$ derived from this work suggest that the production of ${ }^{33} \mathrm{~S}$ could be reduced by a factor of $\sim 12$ over that predicted in the model of José et al. (2001). Such a reduction would clearly affect the ${ }^{32} \mathrm{~S} /{ }^{33} \mathrm{~S}$ ratio to be expected in ONe nova presolar grains. Finally, ONe nova production of ${ }^{34} \mathrm{~S}$ can only occur through the $\beta$-decay of ${ }^{34 m, g} \mathrm{Cl}$, which depends on the aforementioned unknown rates; while ${ }^{34} \mathrm{~S}$ destruction is determined by its own unmeasured destruction rate via ${ }^{34} \mathrm{~S}(p, \gamma){ }^{35} \mathrm{Cl}$. This rate, too, is presently modelled using the Hauser-Feshbach formalism (Iliadis et al., 2001). To exploit any future measurements of sulfur isotopic ratios in $\mathrm{ONe}$ nova candidate grains requires that those measurements can be interpreted within the framework of ONe nova models.

Thus, it is essential to begin experimental efforts to measure and/or constrain these $(p, \gamma)$ reaction 
Table 4.1. ${ }^{34} \mathrm{Ar}$ states and resonance strength and yield estimates. Theoretical and experimental data were collected from Endt (1990), Endt and Firestone (1998), and Iliadis (1997).

\begin{tabular}{cccccccc}
\hline${ }^{34} \mathrm{~S}$ Mirror & $J_{r}^{\pi}$ & $E_{x}$ & $E_{r}$ & $\Gamma_{\gamma}(\mathrm{meV})$ & $\Gamma_{p}(\mathrm{meV})$ & $\omega \gamma(\mathrm{meV})$ & Yield $\left(\mathrm{day}^{-1}\right)$ \\
\hline 4889 & $2^{+}$ & 4865 & 201 & $>16$ & $<5.7 \times 10^{-4}$ & $<3.6 \times 10^{-4}$ & 20 \\
5228 & $0^{+}$ & 4967 & 303 & 25 & $2.9 \times 10^{-3}$ & $3.6 \times 10^{-4}$ & 10 \\
5322 & $2^{-}$ & 5225 & 561 & 25 & 38 & 9.5 & $1.2 \times 10^{5}$ \\
5689 & $5^{-}$ & 5310 & 650 & $1.9 \times 10^{-3}$ & $8.5 \times 10^{-2}$ & $2.6 \times 10^{-3}$ & 28 \\
5381 & $1^{+}$ & 5542 & 878 & 10 & 8500 & 3.8 & $3.1 \times 10^{4}$ \\
\hline
\end{tabular}

rates. Limited information exists for the excited states in both ${ }^{34} \mathrm{Ar}$ that are of interest for this study. For example, only crude upper and lower limits on the decay branches of the 4865, 4967 and $5225 \mathrm{keV}$ states in ${ }^{34} \mathrm{Ar}$ exist (Grawe et al., 1974). Much more information on the ${ }^{34} \mathrm{Ar}$ mirror nucleus ${ }^{34} \mathrm{~S}$ exists and this information can be used to make some estimates for the resonance strengths for the states of interest in ${ }^{34} \mathrm{Ar}$. Table 4.1 shows a listing of the ${ }^{34} \mathrm{Ar}$ levels of interest along with their associated $T=1$ mirror counterparts in ${ }^{34} \mathrm{~S}$.

CRYRING@ESR will offer a unique opportunity to address the $(p, \gamma)$ reactions of interest. Intense beams of radioactive nuclei will be slowed down in the ESR and then transferred to CRYRING where they will be slowed down to the required energy of below $1 \mathrm{MeV} / \mathrm{u}$. Since we aim at measuring resonant reaction rates, the energy of the beam has to be well defined, which will be done by the electron cooling available in the CRYRING. The existing internal gas-jet target will be employed as a windowless hydrogen target. Analogous to the experiment performed in ESR on the ${ }^{96} \mathrm{Ru}(p, \gamma){ }^{97} \mathrm{Rh}$ reaction (Mei et al., $2015)$, the $(p, \gamma)$ reaction products can be intercepted by a particle detector with about $100 \%$ detection efficiency. Particle detectors will have to be placed in the CRYRING ultra-high vacuum. Suitable detectors are being presently prepared for ring experiments at FAIR. Their possible employment in CRYRING will facilitate their successful development. The radiative recombination rate in the target will be used for the in situ luminosity monitor.

The experimental studies will be started with stable $\mathrm{S}$ or $\mathrm{Cl}$ isotopes to establish the technique before going to exotic nuclei. The yields for the ${ }^{33} \mathrm{~S}(p, \gamma){ }^{34} \mathrm{Cl}$ reaction of interest in Table 4.1 were determined assuming a stored ${ }^{33} \mathrm{Cl}$ beam of $10^{6}$ atoms circulating at $100 \mathrm{kHz}$ frequency and assuming $100 \%$ detection efficiency of the ${ }^{34} \mathrm{Ar}$ recoils. 


\section{Chapter 5}

\section{Instrumentation and new techniques}

\$Id: instrumentation.tex,v 1.34 2016/04/25 11:19:31 lestinsk Exp \$

In Chapter 2 - Chapter 4, we have highlighted a rich research programme in atomic and nuclear physics which may realistically keep CRYRING@ESR busy for decades. Pursuing the exciting new opportunities simultaneously requires a set of novel installations for probes and targets, for radiation and recoils detectors, and for quantifying small numbers of stored ions produced through exotic processes. Hence, this chapter will focus on relevant technical aspects and developments. We first give a description of available and planned infrastructure of the CRYRING sections dedicated to experiments (Section 5.1). Then, a discussion of vital experimental instrumentation follows, i.e., detectors for radiation from the visible to x-ray regime (Section 5.2 and 5.3), new targets (Section 5.4) and beam probes, and detectors for nuclear fragments (Section 5.5). Moreover, as the precise determination of cross sections and spectroscopy inherently depend on the ability to quantify the energy and the number of stored ions, in particular, at low, coasting ion currents, latest developments for a new cryogenic current comparator (Section 5.6) and laser-based velocimetry (Section 5.7) are highlighted. Finally, and in preparation for FLAIR, an extraction of the stored ion beam from CRYRING towards the even lower-energetic storage ring Frankfurt Low-energy Storage Ring (FLSR) is sketched in Section 5.8.

The subsequent descriptions largely outline the new experimental installations briefly and summarize their key parameters and specific design choices. For some of these installations already first steps of realization have already been made, and their details were given in corresponding technical design reports (Andelkovic et al., 2015; Kraft-Bermuth et al., 2014; Hengstler et al., 2013).

Even though we do not touch on the specifics of their experiments, one may also mention research programs pursued elsewhere, where synergies with CRYRING@ESR may be utilized. One of them would e.g. be Fast-Ion Slow-Ion Collisions (FISIC) as a French and German cooperation at Spiral2 (Lamour et al., 2010). Parts of their setup is generally mobile and may be transported to Darmstadt for joint campaigns.

\subsection{Target section}

One of the straight sections in CRYRING, 'YR09', is used for installation of targets. The nature of the targets is not restricted, as long as the performance of the storage ring is not compromised. One candidate is the original gasjet target section of CRYRING (Schmidt et al., 1997), but MOTReMi experiments (Section 2.2.5), XUV lasers (Section 2.1.6), and transverse electron targets (Section 5.4) for storage 


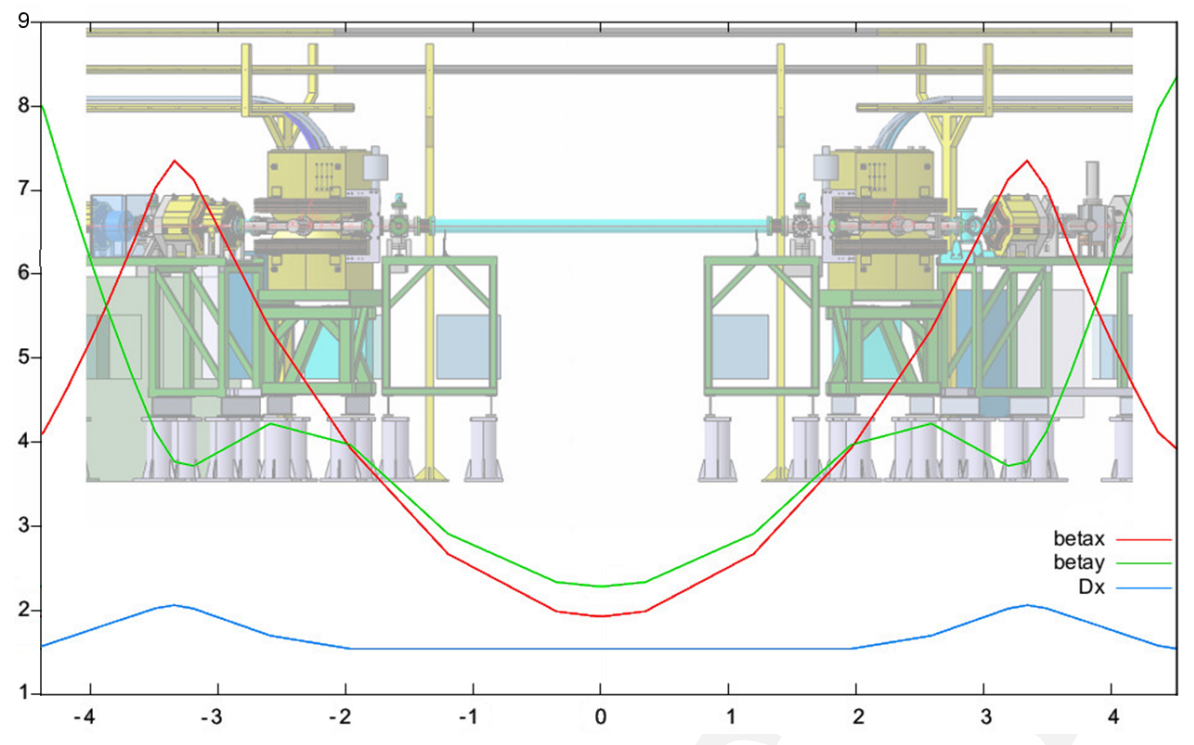

Figure 5.1. Ion beam parameters for one superperiod of CRYRING, centered around the middle of a nonmagnetic section (Gorda et al., 2015). The horizontal and vertical $\beta$-functions are shown with red and green curves, respectively, the dispersion using a blue curve. All units are in meters. The shaded background image is projection of the $3 \mathrm{D}$ model of this segments and aligned to scale.

rings are under consideration as well. Generally, experimentalists may devise any kind of setup to be installed in this section, provided the proposed setup meets the following criteria:

The beam axis is at a nominal height of two meters. The maximum length of the setup is limited to $\approx 3.43 \mathrm{~m}$. Laterally, the experiments may extend up to $1.2 \mathrm{~m}$ from the beam axis and the total height is limited to $2.8 \mathrm{~m}^{1}$. The ring vacuum is segmented and two CF100 gate valves on the entrance and the exit of this section are part of the ring vacuum control system. The experiments can utilize the volume between these valves under the following conditions. In particular, the section must be equipped with sufficient pumping capabilities in order to reach vacuum levels of below $10^{-11}$ mbar by itself. Standard pumping segments are available and may be integrated into the experimental setup. Alternatively, the experiments need to provide their own systems if the standard pumping units are incompatible with the demands of the experiment. Within the section, one CF40 flange must be foreseen for a UHV pressure gauge which is known to and characterized by the GSI vacuum experts and will be integrated into the vacuum control system. The specifications for providing a baking infrastructure in the experiment section are still under discussion. The closed orbit of the circulating stored ion beam must not be destroyed through uncompensated magnetic or electric fields. Moreover, the acceptance of the storage ring must not be compromised by any obstructions in the beamline. As the CRYRING-cave will be in general inaccessible during beam operation, experiments need to be designed with sufficient remote control of all sensible parameters.

The beta function and dispersion for one superperiod are plotted in Figure 5.1 for the design values of the ring tunes $Q_{x}=2.42, Q_{y}=2.42$. The minima of both, horizontal and vertical beta functions and of the dispersion lie at the centers of the non-magnetic sections. Hence, they are also the ideal positions for targets, as the stored beams will have their narrowest envelopes in these positions.

Particle detectors for 'beam-like' products at the target sections are planned at various positions (Andelkovic et al., 2015). 'Beam-like' refers to particles produced from coasting ions which have altered

\footnotetext{
${ }^{1} \mathrm{~A} 3 \mathrm{D}$ model of the installation space can be provided in STEP file format.
} 


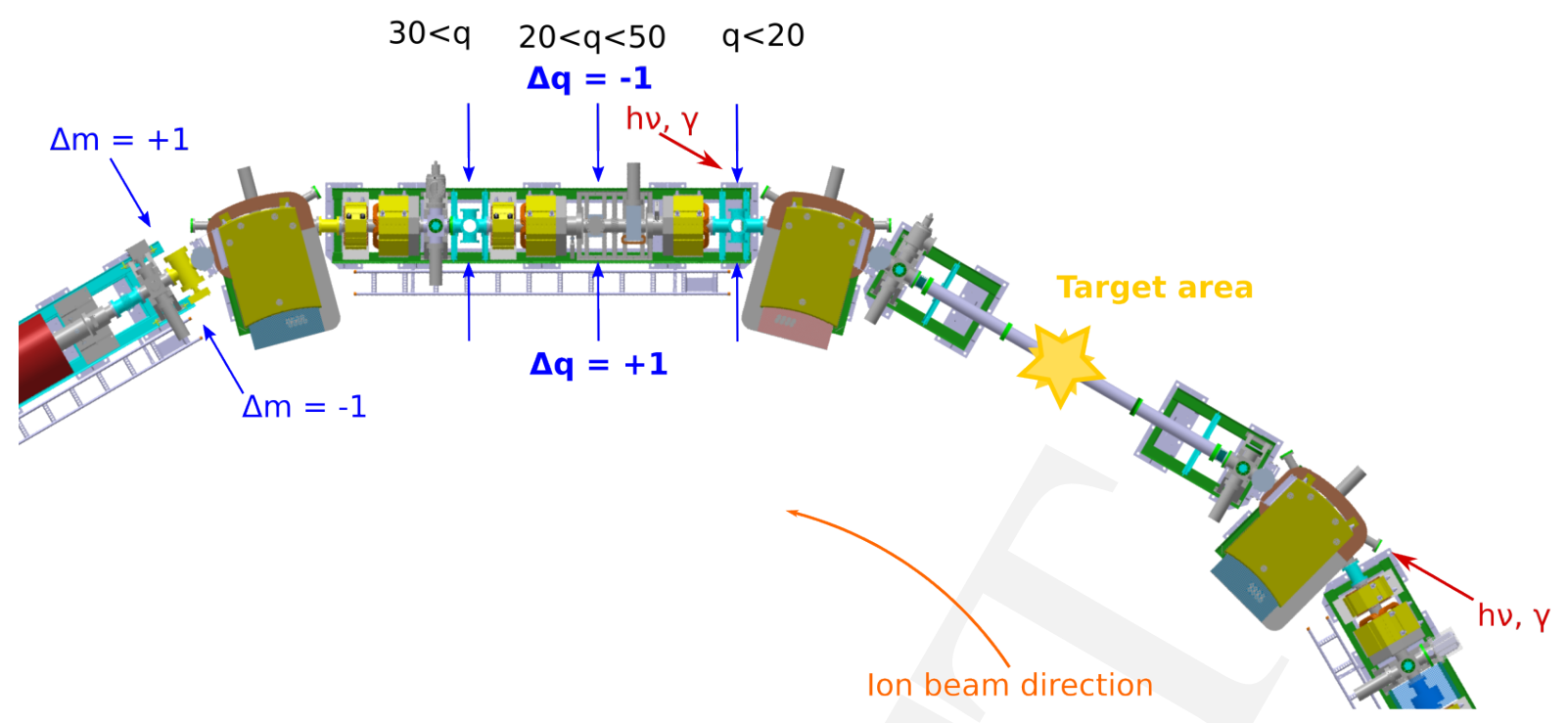

Figure 5.2. Positions of the particle detectors for a segment of the ring with its instrumentation, This layout covers heavy reaction products from the full range of primary ions, from the lightest $p^{+}$to the heaviest $\mathrm{U}^{92+}$, planned at CRYRING@ESR. Shown is a segment of the ring around the target section, YR09, the arrangement after the electron cooler will be similar.

their charge or mass through reactions in the target zone. As many of these processes impose only a negligible momentum transfer onto the involved projectile, these will continue their initial trajectory largely unaltered from the stored parent beam. A separation of these product particles only occurs through the next ring dipole magnets. Figure 5.2 shows the foreseen detector positions which cover the full range of $\Delta q= \pm 1$ reactions from the lightest ions in low charge states to the heaviest highly charged ions.

\subsection{Development of a VUV-VIS-Spectrometer for Target Characteri- sation}

The interaction of HCI beams with atomic or molecular beams (Rzadkiewicz et al., 2006; Gumberidze et al., 2010, Section 2.1.1) or free electrons (Section 2.1.3 Gumberidze et al., 2005; Banaś et al., 2015) has been successfully studied by means of $\mathrm{x}$-ray spectroscopy in order to investigate fundamental interactions such as, e.g., radiative electron capture and its time-reversed counterpart, i.e., the photoelectric effect, in highly charged systems. In addition, beam-foil spectroscopy in the x-ray regime has proven to be a powerful tool for the measurement of dipole transition rates and corresponding lifetimes of excited states (Toleikis et al., 2004, Section 2.1.4).

However, a variety of decay pathways of highly-excited states in atoms and molecules, namely direct fluorescence and autoionization into radiative states (Ehresmann et al., 2004b, 2006), end in excited fragments (Ehresmann et al., 2004b), which by themselves subsequently emit one or more fluorescence photons in the range from visible to vacuum-ultraviolet light, a spectral range that is currently not covered by fluorescence spectrometers at the GSI. Decay pathways producing excited states occur commonly as a result of an ion impact on particle targets. A study by means of fluorescence spectroscopy will give insights into formation processes, not only of specific ions or fragments but also of the electronic states of these reaction products due to the inherent state-selectivity of the technique. The possibility to identify the 
charge state of an ion via its fluorescence spectrum offers quantitative information on multiple-electron transfer during the interaction between the ions and the target: the recorded fluorescence intensity is a measure for the population probability of the fluorescence transition's initial state.

Core-hole formation in target gases via electron transfer onto heavy stored HCI beams is an attractive opportunity for studying the dynamics of the relaxation processes via Yrast cascades in such multiply excited states. HCI collisions for the core hole formation penetrate to the deepest atomic levels, such as for example in the $\mathrm{K}$ shell of xenon, and thus give access to energy regimes beyond what is presently technologically feasible via photoionization at free-electron laser (FEL) and synchrotron radiation beam lines. Moreover, processes of electron transfer, inner-shell excitation or ionization are not restricted by the dipole selection rules known from photonic mechanisms and, thus, have the potential to access entirely different atomic levels. An analysis of electronic states that have been populated in such a process will give data on the decay of these states and thus on electron dynamics in these systems. These experiments require a strongly collimated beam of ions with a low momentum spread as it can be achieved at the CRYRING facility. Due to energy-dependent interaction cross sections, the explanatory power of the recorded data is improved by a small momentum spread and the collimation allows for a sharp imaging of the interaction volume.

A further application is the study of the enhancements of electron-ion recombination at low energies (Gao et al., 1995; Uwira et al., 1997b; Gwinner et al., 2000; Shi et al., 2001, 2002) that has not been fully understood theoretically and can be performed by the spectroscopy of emitted photons arising from dielectronic recombination and radiative recombination. For bare and few-electron low- $Z$ ions, the varying magnetic guiding field along the ions' path through the electron cooler appears to lead to an additional electron capture process (Hörndl et al., 2005; Hörndl et al., 2006). First qualitative experimental investigations support this theoretical model (Lestinsky, 2007). However, for high- $Z$ ions with complex electronic shells, the field-induced rate enhancement does not appear to fully explain the discrepancies of calculated and measured recombination rates with factors of up to three orders of magnitude (Uwira et al., 1997a; Hoffknecht et al., 1998; Schippers et al., 2011; Spruck et al., 2014). Spectroscopy of the emitted recombination photon and a polarization analysis may shed new light on the dynamics of the mechanisms at play, here.

To illustrate the potential of fluorescence spectroscopy, an example is shown in Figure 5.3 for fluorescence from $\mathrm{Kr}$ II formed by photoexcitation followed by autoionization from neutral $\mathrm{Kr}$. There, energies for individual autoionizing Rydberg states of two-electron excitations have been determined after excitation by monochromatized synchrotron radiation.

Every emission cross-section function for a particular fluorescence wavelength corresponds to the final state of an autoionization channel which at the same time is the initial state of the fluorescence transition. With the channels being separated by their fluorescence wavelength, an individual determination of Rydberg states energies has been enabled which is completely impossible in absorption experiments due to the high density of all doubly excited Rydberg states contributing to the absorption signal (Ehresmann et al., 2004a).

\section{Experimental setup and status of the project}

In order to overcome the gap in the fluorescence detection range and to gain information on the aforementioned processes, the set-up of a fluorescence spectrometer for use at the ESR and CRYRING is planned 


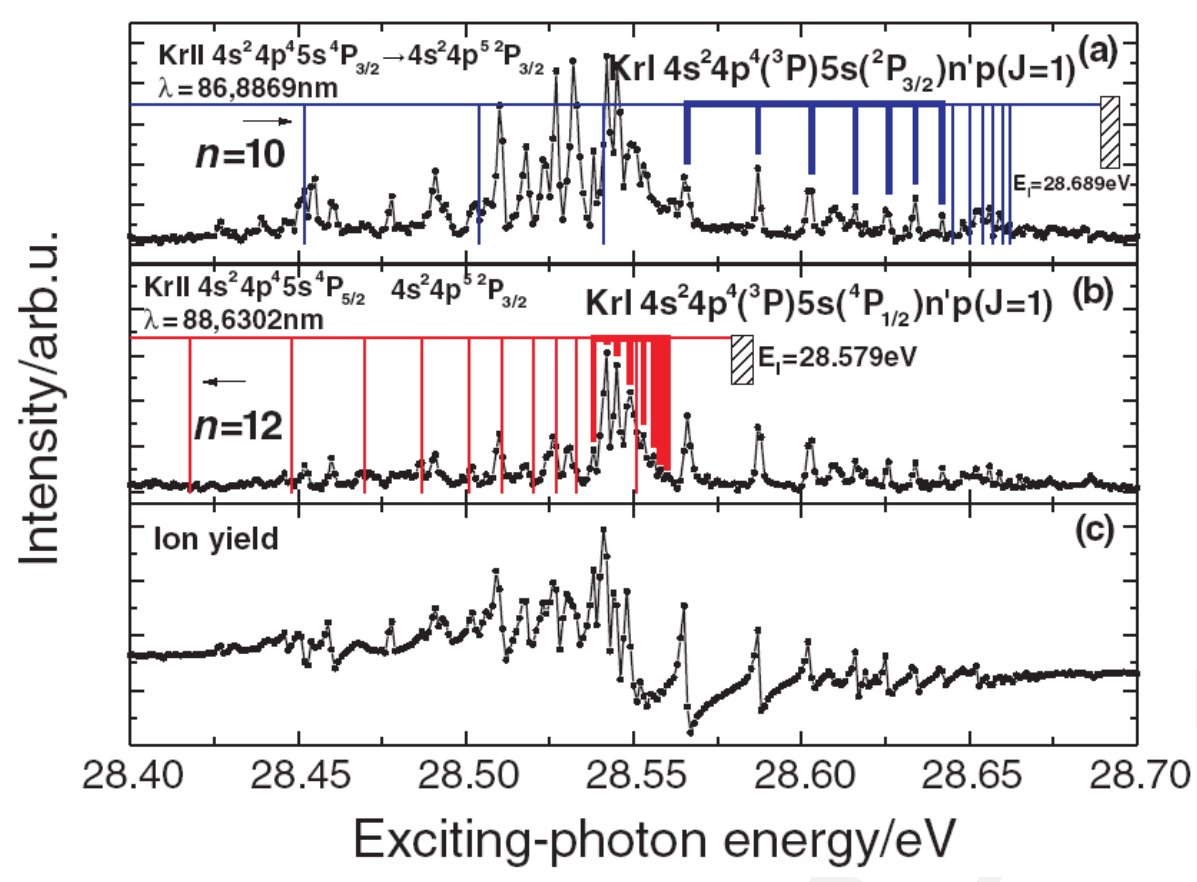

Figure 5.3. Dispersed fluorescence intensities from excited $\mathrm{Kr}$ II $4 s^{2} 4 p^{4} 5 s{ }^{4} P_{3 / 2}$ (a), and $4 s^{2} 4 p^{4} 5 s \quad{ }^{4} P_{3 / 2} \quad$ (b), and satellite states after photon excitation with energies from $28.40 \mathrm{eV}$ to $28.70 \mathrm{eV}$ as well as the total photoion yield (c). Rydberg series of autoionizing two-electron resonances are clearly visible and can be distinguished. (Figure taken from Ehresmann et al., 2004b)

which is capable of covering the demanded fluorescence wavelength range (see Figure 5.4). The setup consists of a McPherson $1 \mathrm{~m}$-normal-incidence spectrometer that can be equipped with interchangeable diffraction gratings for the dispersion of the fluorescence radiation ensuring optimized radiation detection in the vacuum ultraviolet (VUV) and visible light (VIS) spectral ranges. There is no transparent material for the EUV, and only limited transparency in the VUV. Thus, the corresponding spectroscopic arrangement can only use reflective optics, with as few components as possible.

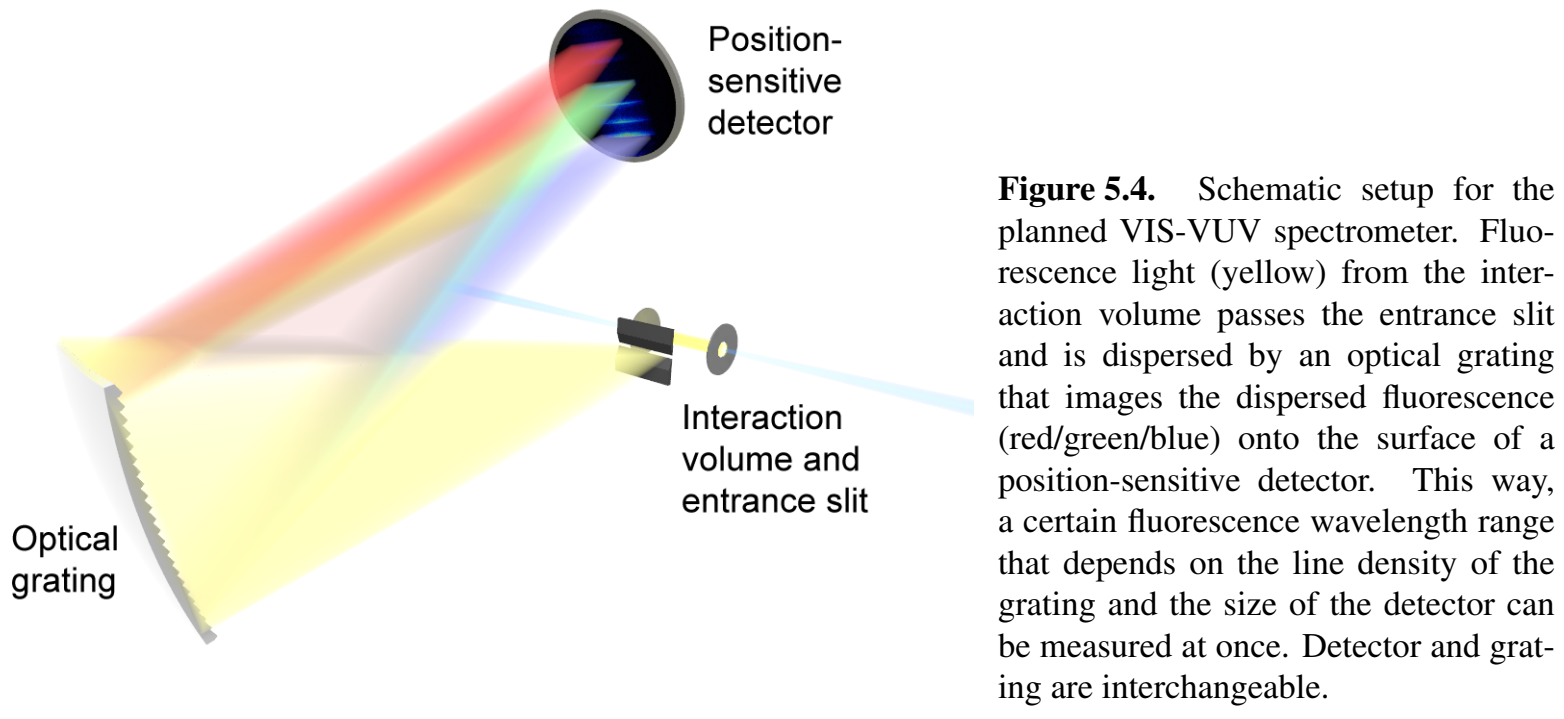

The detection of the photons is performed by 2-dimensional position- and time-resolving singlephoton detectors that allow the simultaneous measurement of several fluorescence lines within a certain fluorescence wavelength range. A time resolution of approximately $300 \mathrm{ps}$ for the photon detection offers the option for lifetime or coincidence measurements in reference to the bunch pattern which helps in reducing the noise caused by random events. In order to further increase the insight in ion and fragment formation after highly charged ion impact, an analysis of the polarization of the fluorescence radiation 
is planned. For the visible fluorescence range down to $300 \mathrm{~nm}$, it is possible to place a Wollaston prism in the path of the dispersed light and splitting the linear horizontal and vertical part of the fluorescence while still being able to detect both parts on the detector simultaneously. However, shorter wavelength ranges are excluded from this method due to the transmission properties of the required materials. Two detectors with wavelength ranges of $190 \mathrm{~nm}$ to $700 \mathrm{~nm}$ for the visible and $115 \mathrm{~nm}$ to $300 \mathrm{~nm}$ for the ultraviolet (UV) and VUV spectral range from Quantar Technology are currently tested and adapted to the system. A third detector for the EUV spectral range from $30 \mathrm{~nm}$ to $150 \mathrm{~nm}$ was developed at the University of Kassel and will be tested after completion.

\subsection{Micro-calorimeters for x-ray spectroscopy}

The development of calorimetric particle detectors operated at temperatures below 1 Kelvin started about 30 years ago. Ever since, one of the driving forces for this development work has been to combine the excellent resolution of crystal spectrometers (which have a very limited wavelength acceptance range) with the large acceptance range of $\mathrm{Ge}$ - or $\mathrm{Si}(\mathrm{Li})$-detectors (which have poor energy resolution).

Micro-calorimeters detect the temperature change of an absorber after a photon deposited its energy as heat. The presently leading micro-calorimeter concepts make use of 3 different types of temperature sensors: Superconducting transition-edge sensors (TESs), highly-doped semiconductor thermistors (neutron transmutation doped germanium (NTD) and implanted silicon) and paramagnetic alloys metallic magnetic calorimeter (MMC). The detection principle and the properties of these three techniques are reviewed in detail in Enss (2005). With all three concepts an energy resolution below $3 \mathrm{eV}$ for soft $\mathrm{x}$-rays has been demonstrated and, recently, TES and MMC based micro-calorimeters even achieved an energy resolution of $1.6 \mathrm{eV}$ (full width at half maximum (FWHM)) for x-rays up to $6 \mathrm{keV}$.

For the physics program at CRYRING two detector systems, based on different thermometer concepts will be available: SiM-X, making use of doped silicon thermistors, and maXs, using metallic paramagnetic temperature sensors. In both detector systems a 'dry' ${ }^{3} \mathrm{He} /{ }^{4} \mathrm{He}$-dilution refrigerator provides the necessary operational temperature below 0.05 Kelvin without the need of handling cryogenic liquids. In both systems the relatively small active area of single micro-calorimeters is compensated by operating an array of several tens to hundreds of them in parallel. Compared to ESR, CRYRING is much more compact. In particular the distance between the electron cooler and the nearest bending dipole is short enough to allow for an attractive solid angle and detection efficiency when maXs/SiM-X are positioned in $0^{\circ} / 180^{\circ}$ direction just behind/in-front these dipoles. This arrangement will minimize uncertainties caused by Doppler broadening and Doppler shifts to a minimum.

\subsubsection{Silicon micro-calorimeters - SiM-X}

Compensated-doped silicon thermistors combine an excellent temperature sensitivity with a large dynamic range. In addition, the use of silicon allows for the production of detector arrays by micromachining (Stahle et al., 1996). Silicon micro-calorimeters for hard x-rays have been developed and applied at GSI since about two decades (Egelhof et al., 1996; Egelhof and Kraft-Bermuth, 2005; KraftBermuth et al., 2014). The present detector array contains 32 detector pixels which consist of silicon thermistors, micro-machined from a wafer of silicon and featuring an implanted area as the thermometer (Stahle et al., 1996) Sn or Pb absorbers are glued onto the thermistors with an epoxy varnish (Bleile 


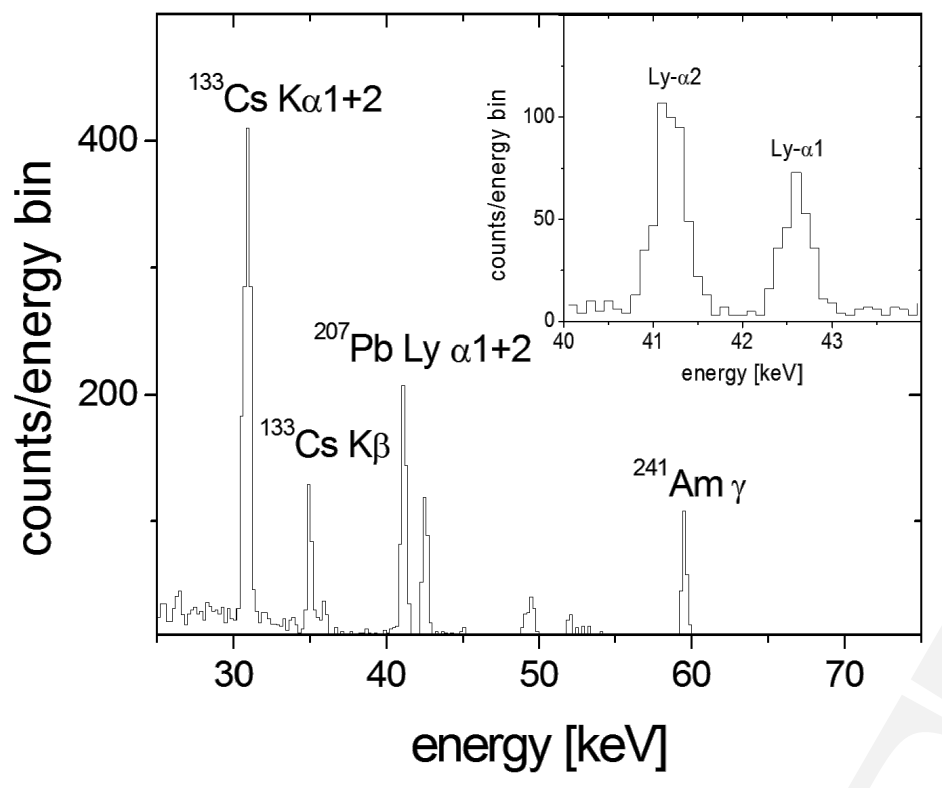

Figure 5.5. Precision X-ray spectroscopy with silicon micro-calorimeters: A spectrum of the 1s Lamb Shift measurement on lead is displayed (Andrianov et al., 2009).

et al., 2002). Each absorber has an active area of about $0.4 \mathrm{~mm}^{2}$ with a photo peak efficiency of $\geq 30 \%$ at $100 \mathrm{keV}$. In the laboratory, an excellent energy resolution of the order of $E \approx 60 \mathrm{eV}$ for $E_{\gamma}=59.5 \mathrm{keV}$ has been achieved.

To obtain a reasonable solid angle, the detectors have to be located as close as possible to the region from which the x-rays are emitted. Accordingly, a special ${ }^{3} \mathrm{He} /{ }^{4} \mathrm{He}$-dilution refrigerator was designed, constructed (Egelhof et al., 1996; Bleile et al., 2002) and applied in experiments (Andrianov et al., 2009; Kraft-Bermuth et al., 2015). This refrigerator is equipped with a side arm which fits to the geometry of the ESR gas-jet target. The micro-calorimeter array is mounted inside the cryostat onto a cold finger at the end of the side arm. In this configuration, the microcalorimeters are kept at an operation temperature of about $50 \mathrm{mK}$. The vacuum chamber of the cryostat is separated from the ultra-high vacuum of the storage ring by windows made of aluminum-coated mylar as well as beryllium or stainless steel. Two detector arrays were applied in two experiments to determine the 1s Lamb shift in hydrogen-like ${ }^{207} \mathrm{~Pb}^{81+}$ in 2006 (Andrianov et al., 2009) and in ${ }^{197} \mathrm{Au}^{78+}$ in 2012 (Kraft-Bermuth et al., 2015). Within experimental uncertainties, good agreement with theoretical predictions was obtained. Both experiments demonstrated the potential of micro-calorimeters to improve the experimental precision. In both experiments, the most significant contribution to the systematic error comes from the correction of the Doppler shift, i.e., from the uncertainty of the exact relative position of gas jet and detector. Doppler broadening from the finite size of gas jet and ion beam, as discussed in Section 2.1.1, contributed to the experimental line width with approximately $50 \%$.

Plans at CRYRING The excellent vacuum conditions in the CRYRING allow for energies well below $10 \mathrm{MeV} / \mathrm{u}$ even for highly-charged uranium. As compared to $100 \mathrm{MeV} / \mathrm{u}$ at the ESR, this will greatly reduce the Doppler shift of the experimental energies and all the systematic errors that come with its correction. It will also reduce the Doppler broadening of the $1 \mathrm{~s} \mathrm{Lamb}$ shift from 100-200 eV to less than $15 \mathrm{eV}$ without reducing the diameter of ion beam or gas-jet target. In such a scenario, the excellent energy resolution of the micro-calorimeters can be exploited to its full extent. Furthermore, as microcalorimeters combine their excellent energy resolution with a large dynamic range, the simultaneous observation of Lyman- $\alpha$ and Balmer lines, which lie at energies around 15-25 keV (Reuschl et al., 2006), is possible. 
The low ion energies at CRYRING provide an advantage in this regard also, because the observation of the Balmer series is much more difficult at high ion energies when they are Doppler shifted to a line energy of about $3 \mathrm{keV}$ or even lower.

As the silicon micro-calorimeter array has already been applied at the internal gas-jet target at the ESR, the setup is well tested. It can be moved to a new experimental site at CRYRING without major modifications. However, further developments are in progress to improve the performance of the detectors and to optimize the cryogenic setup for the application at CRYRING:

- A new ${ }^{3} \mathrm{He} /{ }^{4} \mathrm{He}$-dilution refrigerator with similar design as the refrigerator used in the Lamb shift experiments (Bleile et al., 2002), but running without the supply of cryogenic liquids (KraftBermuth et al., 2012), is currently under commissioning in Giessen. Omitting the use of liquid helium minimizes the interruptions of beam times due to maintenance, because a refilling of the cryostat is not necessary.

- The use of a cryostat without liquid cryogenics permits to mount the micro-calorimeters vertical to the beam line. This geometry is favorable when mounting micro-calorimeters at the electron cooler as is displayed in Figure 2.3.

- The current detector array has 32 pixels which amounts to an active detector area of approximately $10 \mathrm{~mm}^{2}$. To increase the active area, a new detector array with 96 pixels and larger absorbers is currently in preparation.

- The Lyman- $\alpha$ transition energies of highly-charged uranium or gold are of the order of $100 \mathrm{keV}$. Accordingly, the main focus of micro-calorimeter development has been optimization for x-ray energies of 30-100 keV. However, the investigation of hyperfine interactions (Section 2.1.3) or interaction of highly-charged ions with plasmas (Section 2.2.7) calls for micro-calorimeters which are optimized for energies below $5 \mathrm{keV}$. Accordingly, a new detector array will be equipped with very thin absorbers of $\mathrm{HgTe}$ in order to provide high energy resolution for these very low x-ray energies.

At FAIR, the investigation of nuclear and atomic properties of highly charged rare nuclides will be an important part of the experimental program. Of particular interest will be x-ray spectroscopy of rare nuclides. High intensity beams of such rare nuclides will be available from the Super FRS and can be stored in the CRYRING@ESR facility. Then an alternative method to determine absolute nuclear charge radii, namely by measuring the contribution of the nuclear radius to the 1s Lamb shift, can be exploited (Egelhof and Kraft-Bermuth, 2005). Once the QED corrections have been established by measuring the $1 \mathrm{~s}$ Lamb shift in one isotope with known radius, the measurement in a chain of isotopes allows to extract the nuclear radius of each isotope. However, the contribution of the finite size effect to the Lamb shift is of the order of $200 \mathrm{eV}$ for the heaviest nuclides (Stöhlker et al., 2008). To obtain high precision on the nuclear charge radius, high energy resolution is required. A first test case for such an investigation can be tin which has a large number of stable as well as long-lived isotopes. If one of the ion sources at CRYRING be capable to produce bare tin ions, these test experiments can even be performed without the initial need of the GSI accelerator infrastructure.

\subsubsection{Metallic magnetic calorimeters - maXs}

Magnetic calorimeters consist of a metallic absorber for the photons to be detected and a metallic paramagnetic temperature sensor that is placed in a weak magnetic field. Both have a strong thermal link to 

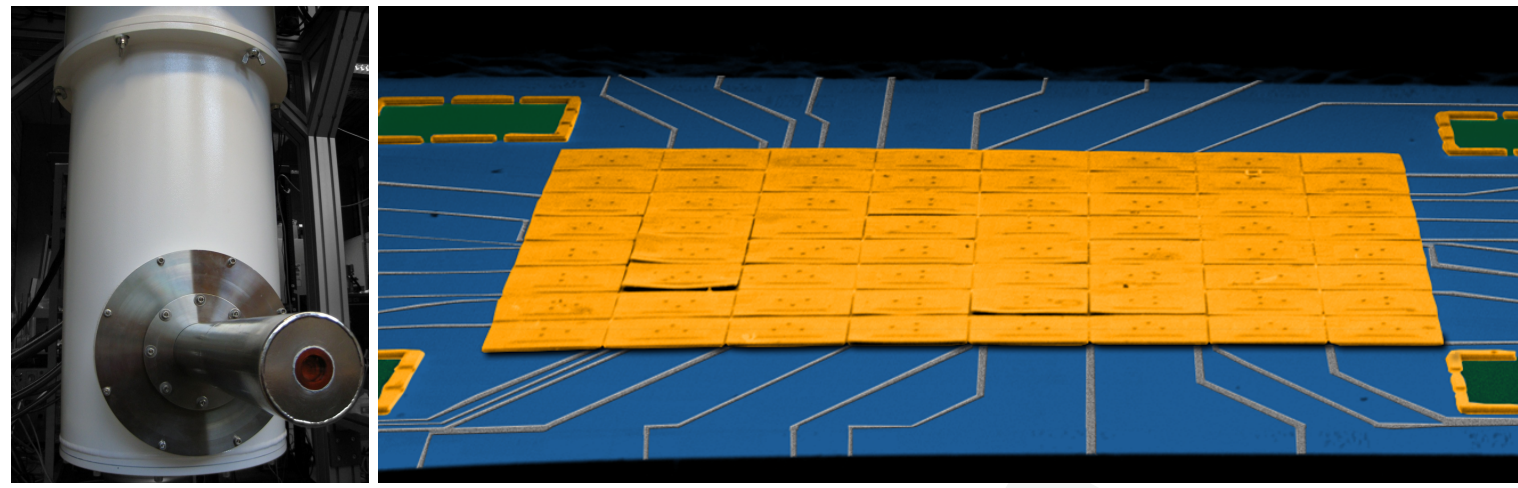

Figure 5.6. Left: Cryogenic platform of maXs. The detector array is operated behind IR-blocking x-ray windows at the end of a $40 \mathrm{~cm}$ long side arm with $80 \mathrm{~mm}$ outer diameter. Right: SEM picture of a maXs-30 detector, an $8 \times 8$ pixel array of micro-calorimeters optimized for $\mathrm{x}$-ray energies up to $30 \mathrm{keV}$.

each other and are weakly coupled to a thermal bath. By monitoring the magnetization of the sensor with a low noise, high bandwidth superconducting quantum interference device (SQUID) magnetometer one obtains an accurate measure of the temperature and therefore of the energy content of the calorimeter.

Micro-calorimeters based on metallic paramagnetic temperature sensors differ from TES- and NTDbased in particular in four points which are of relevance in the context of the experiments planned at CRYRING.

i The intrinsic rise time is 1 to 3 orders of magnitude faster compared to presently discussed TES or NTD detectors, offering coincidence capabilities.

ii There is no abrupt saturation at the upper end of the acceptance range, as exists in the case of TES sensors when the temperature exceeds the transition range.

iii Excellent linearity of the detector response.

iv The properties of the detectors are well explained by standard thermodynamics, which allows for numerical optimization of detector designs for photons of different energy.

The detector system maXs uses a common cryogenic platform (Figure 5.6, left) and a common readout system to operate a variety of $8 \times 8$ arrays of micro-calorimeters, each optimized for a different energy range, i.e. up to $20 \mathrm{keV}, 30 \mathrm{keV}$ (Figure 5.6, right) and $200 \mathrm{keV}$, with energy resolutions of $2 \mathrm{eV}$, $6 \mathrm{eV}, 30 \mathrm{eV}$ (FWHM), respectively, all designed and fabricated at KIP, Heidelberg University (Hengstler et al., 2013). We recently started the development of polar-maXs, a calorimeter-based Compton/Rayleigh polarimeter for $\mathrm{x}$-rays, where the $\mathrm{x}$-rays undergo polarization dependent scattering in dedicated low$\mathrm{Z} /$ medium- $\mathrm{Z}$ scatterers before they are detected by an array of 576 micro-calorimeters with high- $Z \mathrm{x}$-ray absorbers.

In 2014 we performed the first two measurements at the internal gas target of the Experimental Storage Ring at GSI with a metallic magnetic calorimeter of type maXs-200 . In one of these beam times we measured the $\mathrm{x}$-ray spectrum emitted during the radiative recombination of bare Xe ions interacting with a Xe gas jet target, as shown in the Figure 5.7. Here we achieved an energy resolution below $60 \mathrm{eV}$ from $0 \mathrm{keV}$ to $60 \mathrm{keV}$. We were able to detect $\mathrm{K}$-lines from differently charged $\mathrm{Xe}$ ions, including the Lyman series involving high- $n$ levels and could fully resolve the Ly- $\alpha$-doublet in hydrogen-like Xe. The $\mathrm{K}-\alpha$ doublet (as well as the K- $\alpha$ hyper-satellite transitions) produced by double-electron capture events are also clearly visible in the spectrum. A description of the experimental setup and a discussion of the results is provided in Hengstler et al. (2015). 


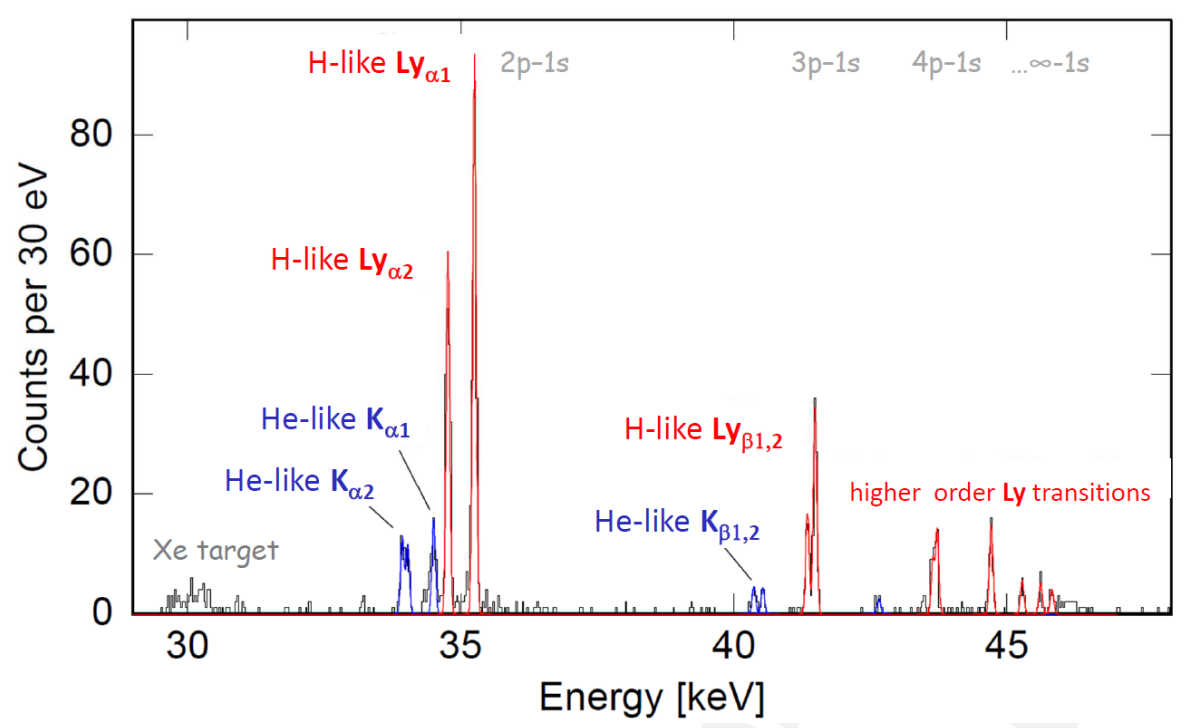

Figure 5.7. X-ray emission spectrum during the radiative recombination of bare Xe-ions (50 MeV/u) interacting with a gas jet of neutral Xe-atoms, measured with maXs-200 at ESR.

\subsection{A free-electron target in transverse collision geometry}

The established approach to perform electron-ion collision spectroscopy at storage rings employs the electron cooler or a cooler-like electron target with a collinear arrangement of electron and ion beam. For CRYRING a transverse-geometry ('crossed-beams') free-electron target is currently being developed by a collaboration of the universities of Frankfurt and Giessen together with GSI (Figure 5.8). Such a target where the electron beam crosses the stored ion beam under an angle of $90^{\circ}$ (see, e.g., Shi et al., 2003; Borovik Jr et al., 2014) is a novel approach for ion storage rings. In contrast to a cooler-like target the interaction region is spatially well localized and facilitates x-ray and electron spectroscopy with relatively large solid angles. Compared to a gas-jet target, providing "quasi-free electrons" in highenergy collisions, the kinematic resolution accessible with a transversal electron target is significantly better, since the electron-energy spread is as low as $0.1 \mathrm{to} 0.2 \mathrm{eV}$ (thermal cathode) as opposed to the $13.6 \mathrm{eV}$ binding energy of a $\mathrm{H}$-target and the resulting Compton-profile.

In a first step an electron density of several $10^{9} \mathrm{~cm}^{-3}$ at a target length of 6 to $10 \mathrm{~cm}$ and an electron energy of the target of up to $10 \mathrm{keV}$ will be realized. Later extensions to electron energies up to 20 $\mathrm{kV}$, to still higher electron densities and to alternative cathode types (field emission or photocathode) are envisaged. A new level of electron-target technology will be reached with the availability of suitable cold beams of polarized electrons.

The new concept of a transversal electron target is ideally suited for CRYRING and offers appealing multifaceted perspectives:

- The use of free electrons in a transversal electron target facilitates photon spectroscopy at low ion energies and thus offers substantially reduced uncertainties due to Doppler shift and Doppler broadening. In this context, the very good vacuum in CRYRING is an important advantage with respect to ion-beam lifetime and detector background.

- Compared to a gas-jet target non-radiative capture (NRC) is absent; at low ion energies ( $\sim 1 \mathrm{MeV} / \mathrm{u})$ usage of a gas-jet target will lead to short beam lifetimes, especially for the highest charge states such as $\mathrm{U}^{92+}$. 

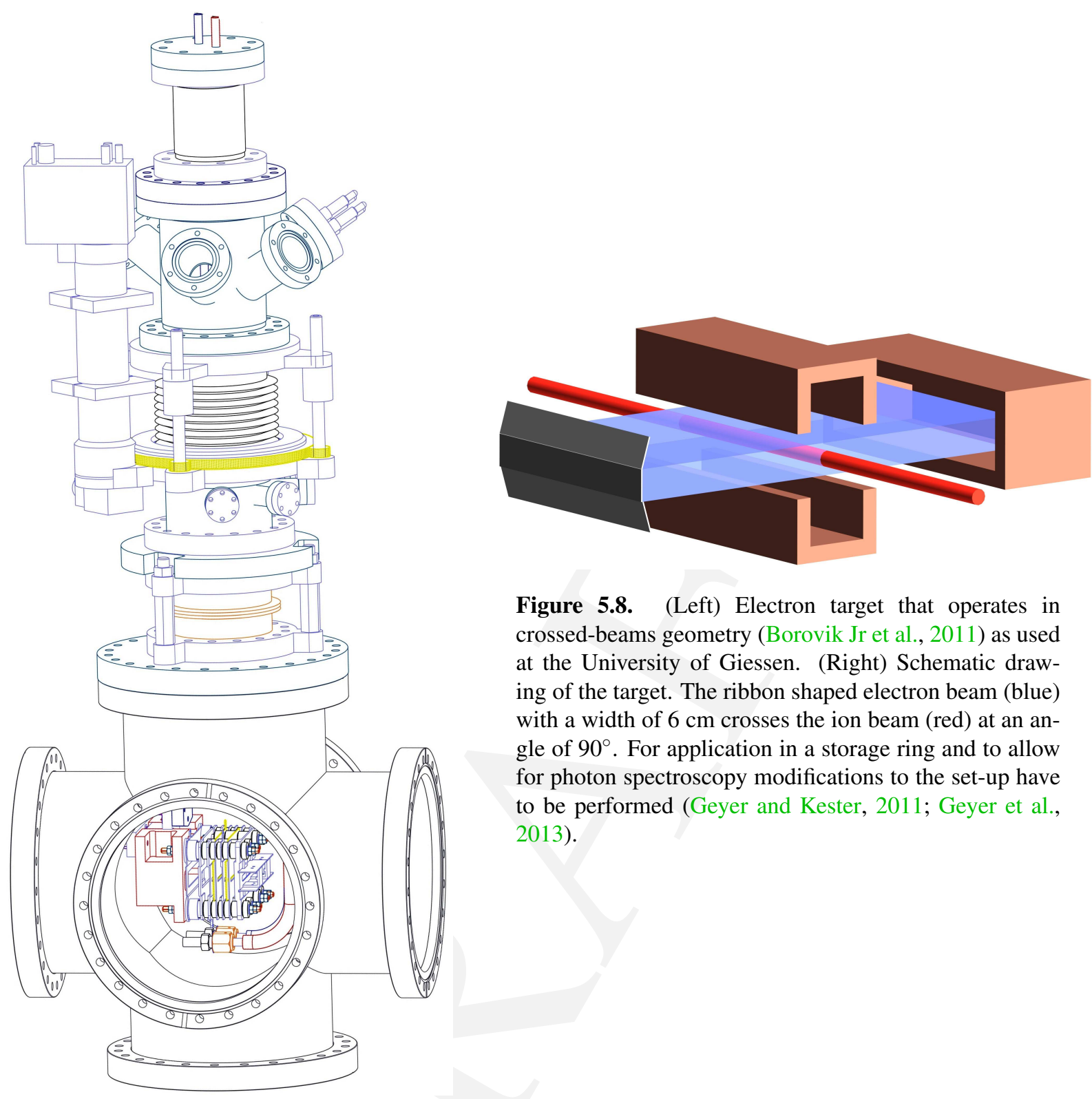

Figure 5.8. (Left) Electron target that operates in crossed-beams geometry (Borovik Jr et al., 2011) as used at the University of Giessen. (Right) Schematic drawing of the target. The ribbon shaped electron beam (blue) with a width of $6 \mathrm{~cm}$ crosses the ion beam (red) at an angle of $90^{\circ}$. For application in a storage ring and to allow for photon spectroscopy modifications to the set-up have to be performed (Geyer and Kester, 2011; Geyer et al., 2013).

- A further advantage of such a transverse free-electron target is the ease of changing the relative collision energy in very fine energy-steps and over a wide range $(\sim 300 \mathrm{eV}-18 \mathrm{keV}$ at the CRYRING). In addition, several combinations of ion and electron energy that lead to the same center-of-mass collision energy can be realized for studying systematic effects.

- In addition, the absence of a target nucleus allows unambiguous electron-impact excitation studies to be performed that are not masked by excitations due the nucleus.

- The combination of an intense mass- and charge-selected cooled ion beam with a transversal electron beam providing a spatially limited target of free electrons, together with high-resolution photon spectroscopy (Section 5.2) where the geometry facilitates large solid angles opens the door to a new level of electron-ion collision studies of excitation and recombination processes. Electronion coincidence techniques will greatly help to eliminate background and to detect correlations between the product-ion charge state and the emitted photon spectrum. 
- Experimental evidence for the yet unobserved NEEC process (Section 3.2) comes into reach by using an approach similar to the one described by Pálffy et al. (2008). The electron energy is sufficient to cover collision energies in the center-of-mass frame of $E_{c m} \approx 15 \mathrm{keV}$, depending on the actual ion energy. This energy is sufficient to even excite specific nuclear transitions and allows for the measurement of the NEEC followed by $\mathrm{x}$-ray emission (NEECX) processes in ${ }^{238} \mathrm{U}^{92+}$ and ${ }^{232} \mathrm{Th}^{90+}$.

- Measurements of the processes of resonant and non-resonant elastic and inelastic electron scattering become possible by combining the transversal free-electron target and high-resolution electron spectroscopy. A particularly attractive subject is the resonant scattering (RS) of free electrons from hydrogen-like ions as an alternative to DR where identical intermediate doubly excited states are populated which then decay by the emission of electrons (RS) or photons (DR). A $10 \mathrm{keV}$ transversal target at CRYRING will facilitate such studies with ions with atomic numbers of below about $Z=40$.

- Studies of resonant and non-resonant pathways in electron-impact ionization of highly charged ions will be performed by observing the ionized ions as a function of the electron-ion collision energy. For such investigations the transverse target, in comparison with a merged-beams geometry, offers the advantage that higher electron-ion collision energies can be accessed. Electron-ion center-of-mass energies of $18 \mathrm{keV}$ are sufficient to study electron-impact ionization of Li-like ions up to about $\mathrm{Xe}^{51+}$ Chen (1992) or Na-like ions up to uranium $\mathrm{U}^{81+}$ Reed et al. (1991) in the interesting ranges of excitation-autoionization and resonant-excitation with subsequent emission of two electrons. The highest charge state accessible so far to such studies has been $\mathrm{Se}^{23+}$ measured at the TSR (Linkemann et al., 1995; Müller, 1995).

\subsection{An UHV compatible detector setup for the investigation of nu- clear reactions at the internal gas-jet target of CRYRING@ESR}

For the investigation of direct nuclear reactions with stored beams, interacting with internal targets of CRYRING@ESR (see Section 4.1) we propose to use an experimental setup which was recently implemented at the internal gas target of ESR, and successfully used for first reaction experiments with radioactive stored beams (von Schmid et al., 2014; Egelhof et al., 2015). The setup (see Figure 5.9), designed as prototype version of the recoil detector for the EXL project consists of an UHV compatible detector chamber which allows to install, besides an internal gas jet target, UHV compatible detectors for target-like reaction products in a relatively large angular range, as well as the necessary infrastructure for performing direct reaction experiments in a storage ring.

At present, two detector units (see Figure 5.9), one consisting of a telescope of a DSSD with an active area of $60 \times 60 \mathrm{~mm}^{2}$, and two $6.5 \mathrm{~mm}$ thick $\mathrm{Si}(\mathrm{Li})$ detectors, the other of only a DSSD, covering the angular range $72^{\circ}<\Theta_{\text {lab }}<88^{\circ}$, and $27^{\circ}<\Theta_{\text {lab }}<38^{\circ}$, respectively, were installed and used for first experiments.

In order to fulfill the strict vacuum conditions in a storage ring a new concept (Streicher et al., 2011; Mutterer et al., 2015) for using Si detector telescopes including their readout and infrastructure in this environment was designed, tested, and recently successfully applied for the first time under running conditions: DSSDs were used as active windows, separating the UHV from an auxiliary vacuum in a 


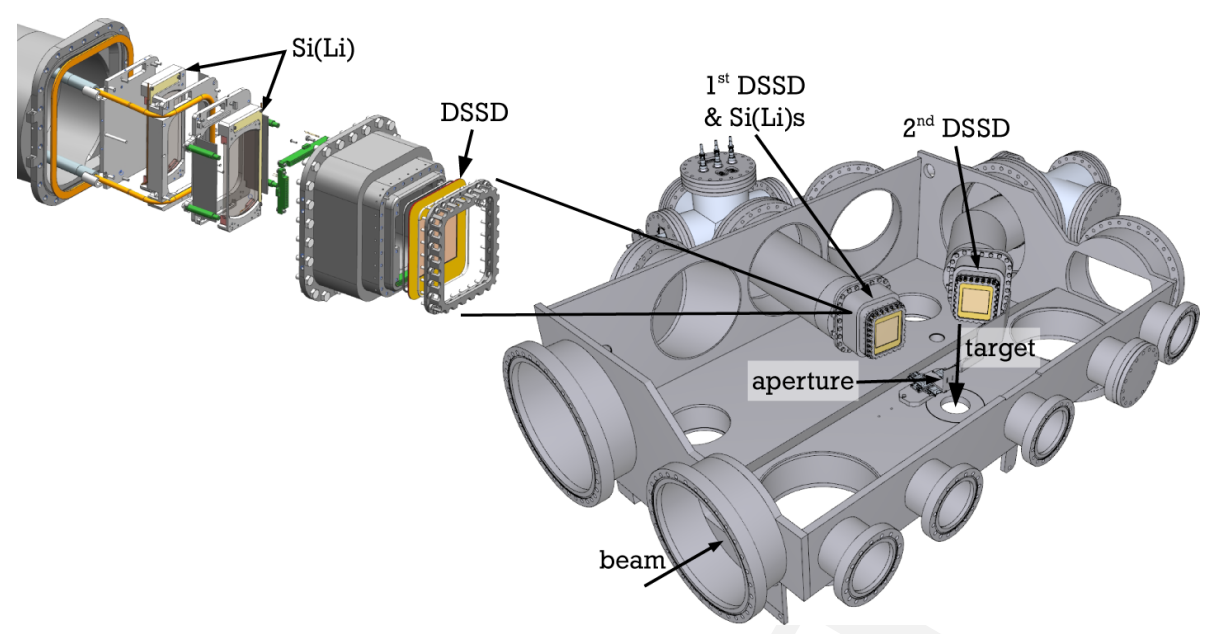

Figure 5.9. schematic view of the EXL setup recently used for reaction experiments at ESR. The total length of the setup in beam direction amounts to about $1.50 \mathrm{~m}$.

vacuum sealed pocket, in which additional $\mathrm{Si}(\mathrm{Li})$ detectors, as well as all cabling for the readout of DSSDs and $\mathrm{Si}(\mathrm{Li})$ s were housed. In addition the $\mathrm{Si}(\mathrm{Li})$ s were cooled during bake-out of the whole setup, as well as during operation. In order to reach the necessary angular resolution required by the experiment, presently limited by the extension of the gas jet target, a remotely-controlled moveable aperture, mounted on two UHV-compatible piezo-motors, was placed in front of the target. Besides improving the angular resolution, this system was also very helpful for determining the actual target position. For an online and off-line energy calibration and functionality test of the DSSDs an Americium $\alpha$-source (not shown in Figure 5.9) could be placed in front of the detection systems.

The present setup was recently mounted at the ESR, commissioned and used for first reaction experiments with stored radioactive beams. As an example, preliminary data for elastic and inelastic proton scattering from ${ }^{56} \mathrm{Ni}$ (von Schmid et al., 2014; Egelhof et al., 2015) are displayed in Figure 5.10 Besides the physics interest, this experiment served also as a proof of principles for the experimental concept of the EXL recoil detector. For the next series of experiments planned at ESR and at CRYRING an upgraded detector setup including 10-20 individual detectors, and thus covering a considerable larger angular range, is presently under design.

\subsection{The Cryogenic Current Comparator for CRYRING@ESR}

This section gives an overview of the $\mathrm{CCC}$ as a non-destructive beam monitor. The CCC consists of a superconducting meander-shaped shielding, a sc pickup coil with a ferromagnetic core, a superconducting matching transformer and a low-temperature direct current SQUID (LTS-DC-SQUID) (see Figure 5.11). The superconducting pickup coil transforms the azimuthal magnetic field of the beam into a current which is measured by a SQUID based current sensor. All other magnetic field components will be highly attenuated by the superconducting shielding. Due to the superconductivity of all parts of the CCC and the use of a SQUID the CCC is able to measure continuous as well as bunched beams. The upper frequency limit is set by the bandwidth of the pickup coil as well as the SQUID electronics. The installation of the CCC requires a ceramic gap in the beam tube like the conventional beam transformers and a liquid helium supply.

The previous CCC was tested in the Horizontal Bi-Cavity Test facility (HoBiCaT) at the Helmholtz- 


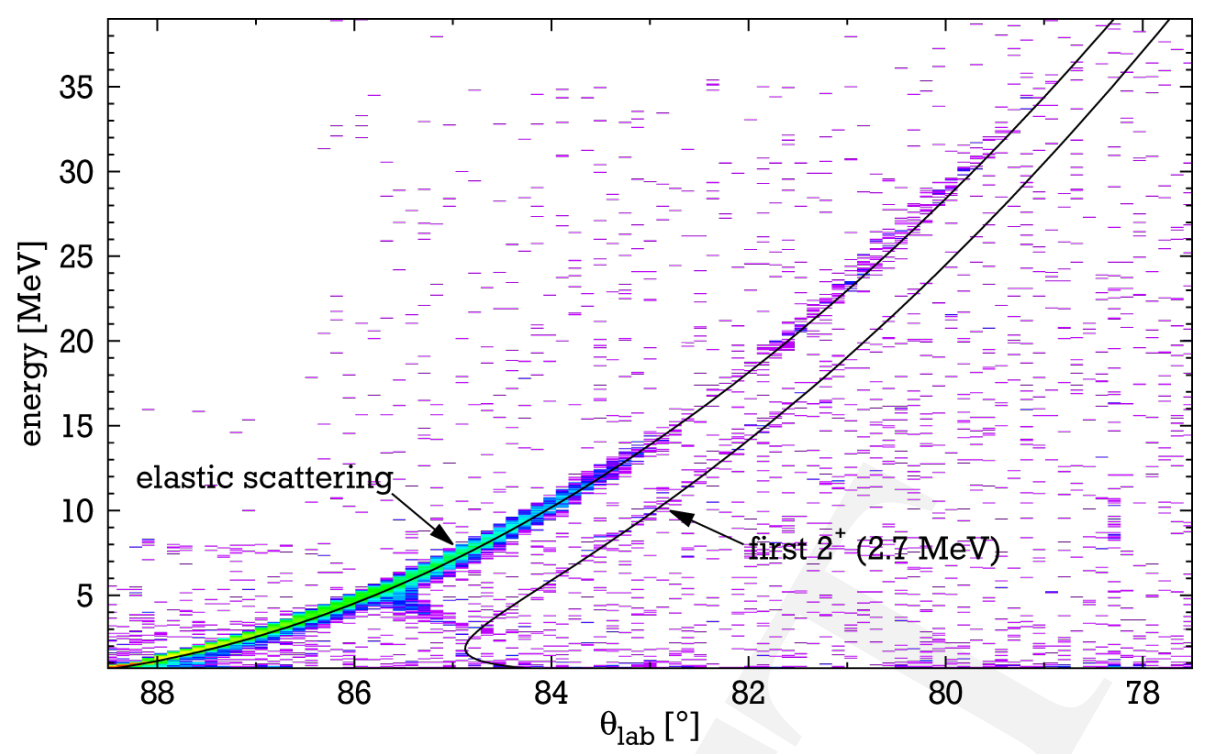

Figure 5.10. The energy of the recoil protons versus the laboratory scattering angle as measured by the detector telescope 1 for $(p, p)$ and $\left(p, p^{\prime}\right)$ reactions on ${ }^{56} \mathrm{Ni}$ in inverse kinematics at $E_{\text {lab }}=400 \mathrm{MeV} / \mathrm{u}$ (von Schmid et al., 2014).

Zentrum Berlin (HZB) as a monitor for dark currents in superconducting cavities in the presence of high gradient electric fields (Geithner et al., 2011). In a quiet laboratory environment the current noise density at $4.2 \mathrm{~K}$ was measured to be $130 \mathrm{pA} \mathrm{Hz}^{-1 / 2}$ at $5 \mathrm{~Hz}$ and $11 \mathrm{pA} \mathrm{Hz}^{-1 / 2}$ at $1 \mathrm{kHz}$. During the test in HoBiCaT the current noise density was increased due to external disturbances like mechanical vibrations. The current noise density at $\mathrm{HoBiCaT}$ at $2.1 \mathrm{~K}$ was measured to be $0.2 \mathrm{nA} \mathrm{Hz}^{-1 / 2}$ at $5 \mathrm{~Hz}$ and increases to $50 \mathrm{nA} \mathrm{Hz}^{-1 / 2}$ in the frequency range from $5-500 \mathrm{~Hz}$ (see Figure 5.12). The real time measurement of a 5 $\mathrm{s}$ bunch of electrons with an intensity of $5 \mathrm{nA}$ was successful (see Figure 5.13). This was achieved by the use of a $10 \mathrm{~Hz}$ low pass filter but without any averaging. The bandwidth of that system was limited to 50 $\mathrm{kHz}$ due to the used core material in the pickup coil and the SQUID electronics working in a modulated flux-locked loop mode.

Figure 5.12. Current noise density of the $\mathrm{CCC}$ in HoBiCaT at HZB.

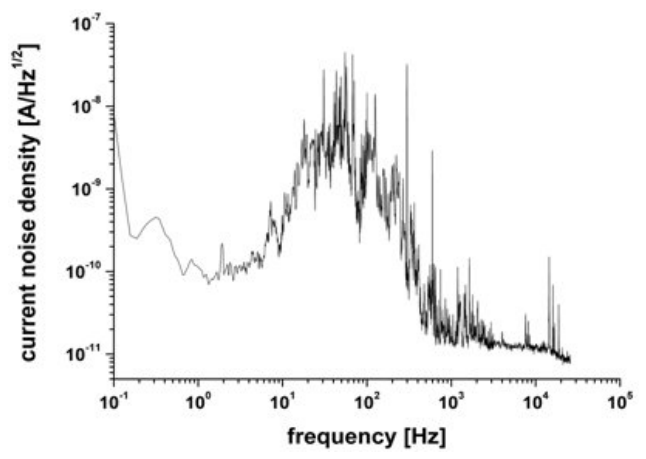

Using SQUID-systems with an extremely low noise level, the sensitivity ultimately depends on the pickup coil with the embedded ferromagnetic core. From intensive investigation on different core materials at low temperatures we found a new material which reduces the current noise density at $4.2 \mathrm{~K}$ to $66 \mathrm{pA} \mathrm{Hz}^{-1 / 2}$ at $5 \mathrm{~Hz}$ and $4 \mathrm{pA} \mathrm{Hz}^{-1 / 2}$ at $1 \mathrm{kHz}$ in a quiet laboratory environment. The total current noise of the pickup coil is reduced by a factor of 3 to approximately $1 \mathrm{nA}$ at $10 \mathrm{kHz}$ bandwidth. The new material also shows a higher bandwidth. Together with a new direct coupled SQUID electronics an overall bandwidth of the $\mathrm{CCC}$ of about $200 \mathrm{kHz}$ is aspired. Some constructional changes will be done 


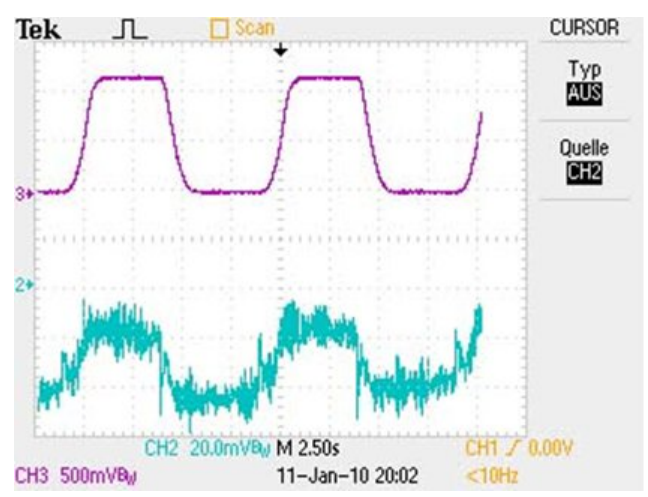

Figure 5.13. Real-time measurement of a $5 \mathrm{~s}$ bunch of dark electrons filtered by a $10 \mathrm{~Hz}$ low pass filter without any averaging (blue). The output signal of the SQUID-electronics of $21 \mathrm{mV}$ corresponds to a dark current of $5 \mathrm{nA}$. The purple curve shows the reference signal of the acceleration field.

to reduce the interference of external disturbances. Dependent on the strength of interference of external disturbances the absolute measurement of $\mathrm{DC}$ as well $\mathrm{AC}$ beam currents down to $1 \mathrm{nA}$ in an accelerator environment should be possible in the real time mode.

\subsection{Laser-based velocimetry and high-voltage measurements}

Laser spectroscopy is a tool for very precise velocity determination of ions. It has been mentioned before that the velocity determination of the ion beam is limiting the accuracy of many experiments. Precision voltage dividers, as they are available at the University of Münster (Thümmler et al., 2009) and have previously been used to measure beam energies in collinear laser spectroscopy experiments (Krieger et al., 2011) will provide means of a voltage determination at the $10^{-5}-10^{-6}$ level at the electron cooler. Laser spectroscopic investigation of transition frequencies of the ions inside the ring can be used to compare the measured voltages with the real ion velocity and to study the influence of experimental parameters like electron-beam ion-beam beam overlap in great detail. Since the transition wavelengths in

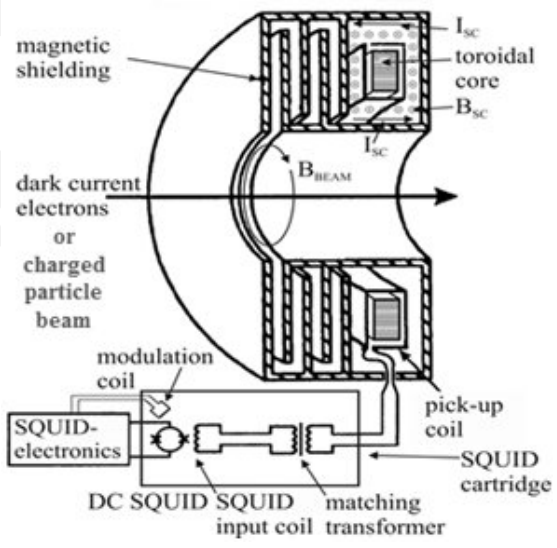

Figure 5.11. Design and working principle of the cryogenic current comparator (CCC) working as a beam monitor $\mathrm{Mg}^{+}$ions is well known (Batteiger et al., 2009), measurements like those described in Section 3.3 inherently generate data for such studies, provided that the laser frequency is measured with sufficient accuracy. This can be realized at different levels of accuracies, e.g., by a high-precision wave meter (Lochmann et al., 2014), laser stabilization to known atomic or molecular transition frequencies (Reinhardt et al., 2006, 2007) or to a frequency comb (Nörtershäuser et al., 2009; Krieger et al., 2012). All techniques have already been used at the ESR or other on-line facilities.

\subsection{FLSR - the electrostatic Frankfurt low energy storage ring}

FLSR is a fully electrostatic storage ring for atomic and molecular ions (Stiebing et al., 2010). The ring has been designed for experiments investigating the dynamics of energetic atomic and molecular reactions. It also serves as a test system for the development of various kinds of novel ion optical 


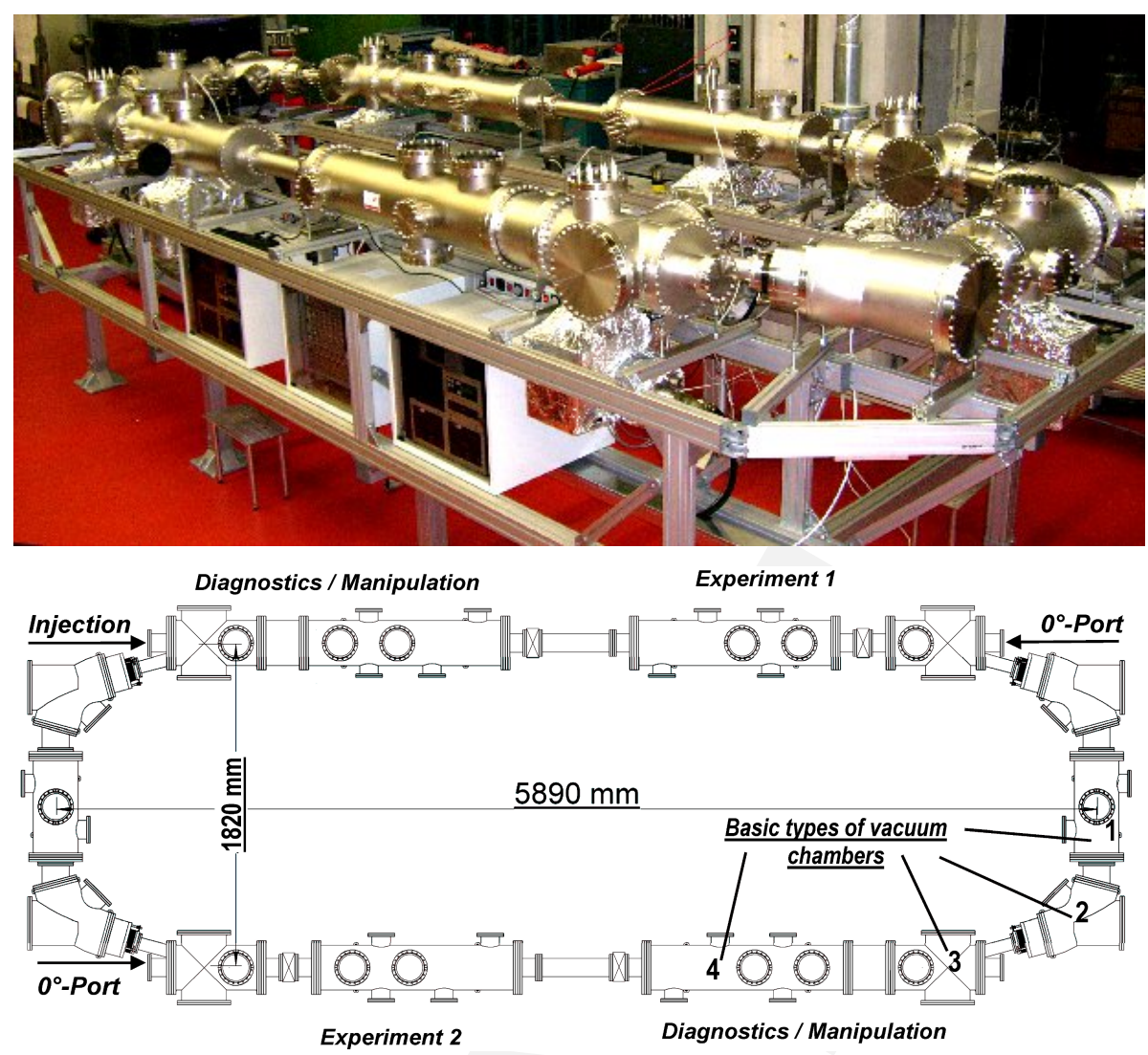

Figure 5.14. Photograph (top) and schematic drawing (bottom) of the FLSR electrostatic storage ring.

devices and diagnostic tools for very low-energy ions. The design energy of FLSR is $50 \mathrm{keV}$ total kinetic energy. Its versatile design (from 4 basic types of vacuum chambers with an inner diameter of $250 \mathrm{~mm}$ ) allows for easy extension of the maximum energy as well as the size of the ring, which presently is restricted to a 'racetrack' geometry of $1.8 \mathrm{~m}$ by $5.9 \mathrm{~m}$ offering two straight sections for experiments and beam manipulation (figure below). A later extension to a symmetric ring with four straight sections for experiments/beam manipulation and diagnostic can easily be accomplished from this design.

As main experimental diagnostic devices two position sensitive multi-channel plate (MCP) detector systems are installed (at the $0^{\circ}$ ports) to observe the neutralized particles. The optical lattice of FLSR has been optimized to provide two points of enhanced ion density in each of the straight sections to allow for sufficient luminosity for differential measurements. FLSR is presently operating at the ion beam facilities of the Institut für Kernphysik Frankfurt (IKF). Besides a transfer beam line from the IKF

Table 5.1. General parameters of FLSR

\begin{tabular}{lrl}
\hline Maximum energy & 50 & $\mathrm{keV}$ \\
Circumference & 14.7 & $\mathrm{~m}$ \\
Revolution time & 4.5 & $\mu \mathrm{s}$ \\
(protons at 50 keV) & & \\
Smallest beam & $3 \times 4$ & $\mathrm{~mm}^{2}$ \\
Tune values Qx & 3.574 & \\
\multicolumn{1}{c}{ Qy } & 2.125 & \\
\hline
\end{tabular}


14-GHz electron cyclotron resonance (ECR) ion source a separate 50-kV HV terminal is presently under construction to allow for the installation of various ion sources. 


\section{Chapter 6}

\section{Summary and Outlook}

\$Id: summary.tex, v 1.14 2016/04/25 11:19:31 lestinsk Exp \$

In this text, we have previewed a research programme for the immediate future of CRYRING@ESR. This new facility offers a unique 'sweet spot' of available ions and isotopes for research with and on slow beams of highly charged ions, up to the ultimate bare $\mathrm{U}^{92+}$. Production of these ions in the GSI accelerator infrastructure leading towards CRYRING@ESR is a routine operation already today and with the new machine obtains access to long-lived storage of intense and brilliant beams at low energy. Thus, we can start with experiments in uncharted territory already from the first day of operation. Examples of such experiments are the precision measurements on atomic structure and dynamics in extremely strong fields, on nuclear structure near the Coulomb barrier and nuclear reactions, on deriving nuclear charge radii, hyperfine interaction, or studying couplings between electronic shell and nucleus. In addition, with the local $300 \mathrm{keV} / \mathrm{u} \mathrm{RFQ}$ injector beamline an option for self-sustaining beam operation exists, even though at a reduced set of available ions.

Furthermore, research beyond atomic and nuclear physics has not even been touched upon in this manuscript. Such would be e.g. materials research with slow, highly charged ions interacting with bulks and surfaces, or the interaction of complex organic molecules with HCI radiation, relevant e.g. for understanding the breakup reactions and chemistry of molecular clouds in interstellar space or in biophysics. Another large field, for which CRYRING@ESR will play an essential role, is the physics with slow and cooled antiproton beams as pursued by the FLAIR collaboration (Widmann, 2015) at FAIR. For the latter, a proposal exists to extend the future FAIR facility, where 'A' stands for "antiprotons", by constructing a dedicated beam line connecting FAIR to the ESR and thus also to CRYRING (Stöhlker et al., 2015; Katayama et al., 2015). In this way high quality antiproton beams will be available in CRYRING for experiments directly in the ring as well as with extracted beams. Thus there is an immense potential even yet to be contrived.

Commissioning of CRYRING with the local ion injector is planned for 2016, and based on the present timeline of FAIR construction, we may begin with injection HCI beams from ESR into CRYRING in 2018, thus deploying the full facility of 'CRYRING@ESR' to the scientific community. The first set of experimental instrumentation of CRYRING@ESR (Andelkovic et al., 2015) has recently received approval and funding for a period of 2015-2018. With these setups, research at CRYRING@ESR may commence in due time for the GSI accelerator facility being brought back into operation in 2018. 
Acknowledgements: We gratefully acknowledge the efforts and contributions of all involved experts and groups who proficiently support the realization of the CRYRING@ESR project from the earliest phases of design to construction and commissioning: Next to the technical divisions at GSI, these are our partners at Stockholm University, at Jagiellonian University Kraków, at KVI Groningen, and at Helmholtz-Institute Jena.

This work was supported in part by the Hesse-State Initiative for Development of Scientific and Economic Excellence (LOEWE) in the LOEWE-Research Cluster Electron dynamics of CHiral systems (ELCH), the Helmholtz-CAS Joint Research Group (HCJRG-108), and the European Research Council (ERC) under the European Union's Horizon 2020 research and innovation programme (grant agreement No 682841 'ASTRUm'). 


\section{Acronyms}

CCC cryogenic current comparator

CCC covergent close coupling

CCD charge-coupled device

DR dielectronic recombination

DSSD double-sided silicon strip detector

DWBA distorted wave Born approximation

EBIT electron-beam ion-trap

ECC electron capture to the continuum

ECR electron cyclotron resonance

ECS exterior complex scaling

EOS equation of state

ESR Experimental Storage Ring

EUV extreme ultraviolet

EXAFS extended x-ray absorption fine-structure

EXL EXotic nuclei studied in Light ion induced reactions at the NESR storage ring http://www.rug.nl/kvi-cart/research/hnp/research/exl/

FAIR Facility for Antiproton and Ion Research in Europe, Darmstadt, Germany http://www.fair-center.eu

FEL free-electron laser

FISIC Fast-Ion Slow-Ion Collisions

http://www.insp.upmc.fr/Collisions-Ion-Lent-Ion-Rapide.html

FLAIR Facility for Low-energy Antiproton and Ion Research http://www.flairatfair.eu/ 
FLSR Frankfurt Low-energy Storage Ring

FRS Fragment Separator

FWHM full width at half maximum

GSI GSI Helmholtzzentrum für Schwerionenforschung GmbH, Darmstadt, Germany http://www.gsi.de/

HCI highly charged ion

HFI hyperfine induced

HHG high harmonic generation

HoBiCaT Horizontal Bi-Cavity Test facility

HZB Helmholtz-Zentrum Berlin

IATI inverse above-threshold ionization

IC internal conversion

IKF Institut für Kernphysik Frankfurt

LTS-DC-SQUID low-temperature direct current SQUID

MCP multi-channel plate

MMC metallic magnetic calorimeter

MO molecular orbital

MOT magnetooptical trap

MOTReMi MOT reaction microscope

MPIK Max-Planck-Institut für Kernphysik, Heidelberg, Germany http://www.mpi-hd.mpg.de/

MS main sequence

NEEC nuclear excitation by electron capture

NEECX NEEC followed by $\mathrm{x}$-ray emission

NEET nuclear excitation in electron transition

NRC non-radiative capture

NTD neutron transmutation doped germanium 
PHELIX Petawatt high-energy laser for heavy-ion experiments

QED quantum electrodynamics

REC radiative electron capture

RECC radiative electron capture to the continuum

RG red giant

$\mathbf{R} \mathbf{R}$ radiative recombination

RS resonant scattering

SEE slow electron emission

SIS18 Schwerionensynchrotron (heavy ion synchrotron) with a maximum rigidity $B \rho=18 \mathrm{Tm}$

SPARC Stored Particles Atomic Physics Research Collaboration http://www.gsi.de/sparc/

SQUID superconducting quantum interference device

SR special relativity

TDCS triple differential cross sections

TES transition-edge sensor

TNR thermonuclear runaway

TSR Test Storage Ring

UHV ultra-high vacuum

UV ultraviolet

VIS visible light

VUV vacuum ultraviolet

WD white dwarf

WDM warm dense matter

XUV extreme ultraviolet 


\section{List of Tables}

1.1 CRYRING@ESR operation parameters $\ldots \ldots \ldots \ldots \ldots \ldots \ldots \ldots$

$4.1{ }^{34} \mathrm{Ar}$ states and resonance strength and yield estimates. . . . . . . . . . . . . 57

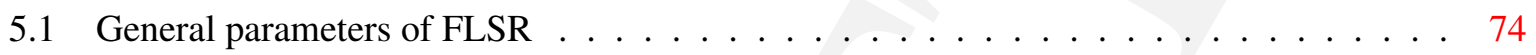




\section{List of Figures}

1.1 Energy ranges and storage rings for highly charged ions at GSI/FAIR . . . . . . . . . 3

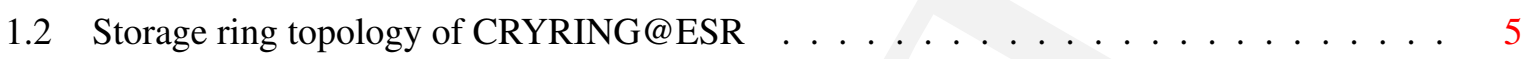

1.3 Top view of the CRYRING model in the new CRYRING@ESR configuration. . . . . . . 6

2.1 Electric field for $1 s$ electrons and comparison of QED contributions $\ldots \ldots \ldots \ldots$

$2.2 \mathrm{X}$-ray spectrum of RR into bare uranium . . . . . . . . . . . . . . . . 9

2.3 Scheme for a possible experimental setup at the electron-cooler of the CRYRING . . . . 10

2.4 Correlation diagram for the symmetric $\mathrm{Pb}-\mathrm{Pb}$ collision. . . . . . . . . . . . . . . 13

2.5 Energy resolution in merged beams experiments for CRYRING and ESR electron coolers 15

2.6 Simulated DR spectra of Li-like ${ }^{142} \mathrm{Nd}^{57+}$ at different electron temperatures . . . . . . . 15

2.7 Linear polarization of hyperfine decay line and $\mathrm{K} \alpha_{1}$ following REC into $1 s 2 p_{3 / 2}{ }^{205} \mathrm{Tl}^{79+} \quad 20$

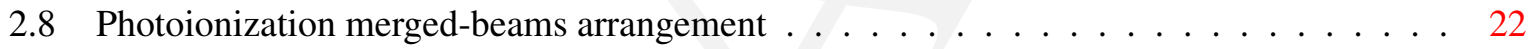

2.9 Measured and calculated photoionization cross sections for $\mathrm{C}^{+} \ldots \ldots \ldots \ldots \ldots$

2.10 Typical HHG spectra for $\mathrm{Xe}$ and $\mathrm{Kr}$ and profiles . . . . . . . . . . . . . . . . . 23

2.11 Schematic coupling section for the XUV beam to the ring. . . . . . . . . . . . . . 24

2.12 e-e correlation in the rest-frame angular distributions of $1 s 2 s^{2} 2 p_{3 / 2}$ autoionization for $Z=54,72$, and 92 , and for $T_{p}=5,10$, and $100 \mathrm{MeV} / \mathrm{u} \ldots \ldots \ldots 25$

2.13 e-e correlation in the lab-frame angular distributions of $\mathrm{U}^{88+} 1 s 2 s^{2} 2 p_{3 / 2}$ autoionization . 26

2.14 Measured and calculated absolute TDCS for $(e, 2 e)$ on He at $E_{0}=70.6 \mathrm{eV} . \quad \ldots \ldots$.

2.15 TDCS for EII of He-like $\mathrm{Fe}^{24+}$ at $16457 \mathrm{eV}$ impact energy in the projectile frame . . . . 29

2.16 Laboratory energy of inelastically scattered electron after ionizing $\mathrm{H}-$ like $\mathrm{Fe}^{25+}$ as function of laboratory observation angle. . . . . . . . . . . . . . . . . . 30

2.17 RECC cusp for $\mathrm{U}^{88+}+\mathrm{N}_{2}$ collisions at $90 \mathrm{MeV} / \mathrm{u} \ldots \ldots \ldots \ldots \ldots$. . . . . . . . 30

2.18 DDCS for electron emission in $1-\mathrm{MeV} \mathrm{p}+\mathrm{Ne}$ and in $0.35 \mathrm{MeV} / \mathrm{u} \mathrm{I}^{23+}+\mathrm{He}$ collisions. . . 32

2.19 The MOTReMi apparatus . . . . . . . . . . . . . . . . . . . . . . . . 33

2.20 Single electron capture in $63 \mathrm{keV} \mathrm{Ne}^{7+}-\mathrm{He}$ collisions. . . . . . . . . . . . . . . 34

$2.21 \mathrm{Ar}$ droplet heating in a intense laser field . . . . . . . . . . . . . . . . . . . 38

2.22 Radiation spectrum of the recombination process . . . . . . . . . . . . . 40

2.23 Scheme for the experimental arrangement in an IATI experiment. . . . . . . . . . . 40

3.1 Nuclear volume shift in DR of Li-like ${ }^{142} \mathrm{Nd}^{57+}$ and ${ }^{150} \mathrm{Nd}^{57+} \ldots \ldots \ldots \ldots \ldots \ldots$. . . . . 42

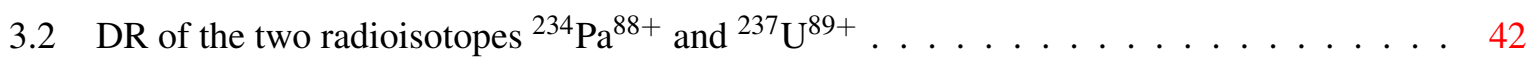

3.3 Known level scheme of ${ }^{129} \mathrm{Sb}$ atoms. . . . . . . . . . . . . . . . . . . . . . . 44

3.4 Schottky frequency spectra of well resolved isobaric doublet $\ldots \ldots \ldots \ldots$. . . . . 45 
3.5 Indication of optical pumping was observed in experiments on a $\Lambda$-scheme of $\mathrm{Li}^{+}$ions with $\beta=0.34$ at the ESR . . . . . . . . . . . . . . . . . 46

4.1 Reaction network during the $p$-process nucleosynthesis between $\mathrm{Nb}$ and $\mathrm{In}$ for $\mathrm{T}_{9}=2.4$. $\quad 50$

4.2 Double Sided Silicon Strip Detector as used to detect $(\mathrm{p}, \gamma)$ inside the ESR . . . . . . . 52

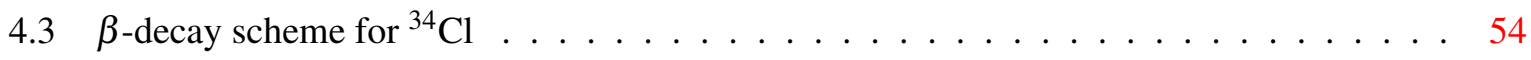

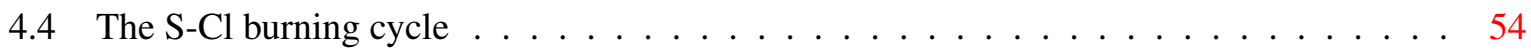

4.5 Level schemes of ${ }^{34} \mathrm{Ar}$ and ${ }^{35} \mathrm{Ar} \ldots \ldots \ldots \ldots \ldots \ldots$

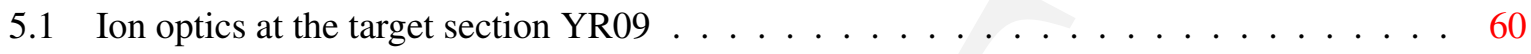

5.2 Foreseen particle detector positions at the target section . . . . . . . . . . . 61

5.3 Fluorescence from doubly excited $\mathrm{Kr}$ II as an example for fluorescence spectrometry . . 63

5.4 Scheme for the VIS-VUV-Spectrometer Setup . . . . . . . . . . . . . . . 63

5.5 Precision X-ray spectroscopy with silicon micro-calorimeters . . . . . . . . . . . . 65

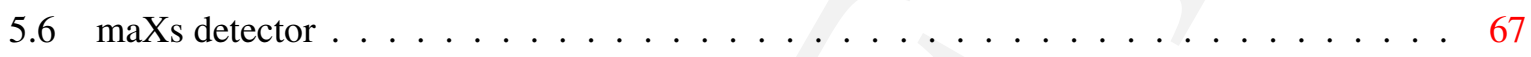

5.7 maXs x-ray spectrum measured in $\mathrm{Xe}^{54+}$ on Xe collisions $\ldots \ldots \ldots$. . . . . . 68

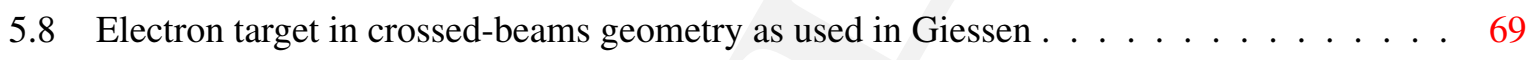

5.9 Schematic view of EXL at ESR . . . . . . . . . . . . . . . . 71

$5.10(p, p)$ and $\left(p, p^{\prime}\right)$ recoil proton energies vs. scattering angles for ${ }^{56} \mathrm{Ni}$ at $400 \mathrm{MeV} / \mathrm{u} \quad \ldots 72$

5.12 Current noise density of the CCC in HoBiCaT at HZB . . . . . . . . . . . . 72

5.13 CCC realtime measurement. . . . . . . . . . . . . . . . . 73

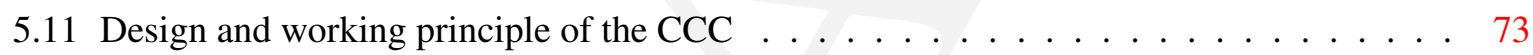

5.14 Photograph and schematic drawing of the FLSR electrostatic storage ring . . . . . . . 74 


\section{Bibliography}

Åberg T (1991): in D Berényi and G Hock (editors), High-Energy Ion-Atom Collisions, volume 376 of Lecture Notes in Physics, 313-324, Springer Berlin Heidelberg, ISBN 978-3-540-53738-0

Abrahamsson K, Andler G, Bagge L et al. (1993): Nucl. Instrum. Methods B 79, 269

Abufager PN, Martínez AE, Rivarola RD, and Fainstein PD (2004): J. Phys. B 37, 817

Ali S, Orban I, Mahmood S et al. (2012): Astrophys. J. 753, 132

Amari S, Gao X, Nittler LR et al. (2001): Astrophys. J. 551, 1065

Andelkovic Z, Brandau C, Dumchev M et al. (2015): Experimental Instrumentation of CRYRING@ESR,

Technical design report, FAIR, Darmstadt, http://www.fair-center.eu/fileadmin/fair/publications_ exp/TDR_CRYRING_Experimental_Instrumentation.pdf

Andersson M, Zou Y, Hutton R, and Brage T (2009): Phys. Rev. A 79, 032501

Andreev OY, Labzowsky LN, Plunien G, and Solovyev DA (2008): Phys. Rev. 455, 135

Andrianov V, Beckert K, Beller P et al. (2008): J. Low Temp. Phys. 151, 1049

Andrianov V, Beckert K, Bleile A et al. (2009): AIP Conf. Proc. 1185, 99

Antognini A, Nez F, Schuhmann K et al. (2013): Science 339, 417

Arnold E, Bonn J, Gegenwart R et al. (1987): Phys. Lett. B 197, 311

Arnould M and Goriely S (2003): Phys. Reports 384, 1

Artemyev AN, Shabaev VM, Tupitsyn II et al. (2013): Phys. Rev. A 88, 032518

Asp S, Schuch R, DeWitt D et al. (1996): Nucl. Instrum. Methods B 117, 31

Bagnoud V, Aurand B, Blazevic A et al. (2010): Appl. Phys. B 100, 137

Banaś DD, Pajek M, Surzhykov A et al. (2015): Phys. Rev. A 92, 032710

Batteiger V, Knünz S, Herrmann M et al. (2009): Phys. Rev. A 80, 022503

Baumann P, Blum M, Friedrich A et al. (1988): Nucl. Instrum. Methods A 268, 531

Beauvoir BD, Schwob C, Acef O et al. (2000): Eur. Phys. J. D 12, 61

Beck BR, Becker JA, Beiersdorfer P et al. (2007): Phys. Rev. Lett. 98, 142501

Beiersdorfer P, Chen H, Thorn DB, and Träbert E (2005): Phys. Rev. Lett. 95, 233003

Beiersdorfer P, Crespo López-Urrutia JR, and Träbert E (2016): Astrophys. J. 817, 67

Bernhardt D, Brandau C, Kozhuharov C et al. (2012): J. Phys.: Conf. Ser. 388, 012007

Bernhardt D, Brandau C, Harman Z et al. (2015a): Phys. Rev. A 91, 012710

- (2015b): J. Phys. B 48, 144008

Berrah N, Fang L, Murphy B et al. (2011): Proceedings of the National Academy of Sciences 108, 16912

Beyer H, Gassner T, Trassinelli M et al. (2015): J. Phys. B 48, 144010

Beyer HF (1997): Nucl. Instrum. Methods A 400, 137

Beyer HF, Menzel G, Liesen D et al. (1995): Z. Phys. D 35, 169

Beyer HF, Stöhlker T, Banas D et al. (2004): Spectrochimica Acta Part B: Atomic Spectroscopy 59, 1535 
Bleile A, Egelhof P, Kluge HJ et al. (2000): Nucl. Instrum. Methods A 444, 488

Bleile A, Egelhof P, Kraft S et al. (2002): AIP Conf. Proc. 605, 409

Bondarevskaya A, Prozorov A, Labzowsky L et al. (2011): Phys. Rev. 507, 1

Borneis S, Bosch F, Engel T et al. (1994): Phys. Rev. Lett. 72, 207

Borovik Jr A, Brandau C, Jacobi J, Schippers S, and Müller A (2011): J. Phys. B 44, 205205

Borovik Jr A, Shi W, Jacobi J, Schippers S, and Müller A (2014): J. Phys.: Conf. Ser. 488, 142007

Bosch F and Egelhof P (editors) (1997): Proceedings of the Third International Conference on Nuclear Physics at Storage Rings, Bernkastel-Kues, 1996, volume 626, Elsevier, http://www.sciencedirect. com/science/journal/03759474/626/1-2

Bosch F, Faestermann T, Friese J et al. (1996): Phys. Rev. Lett. 77, 5190

Botermann B, Bing D, Geppert C et al. (2014): Phys. Rev. Lett. 113, 120405

Brandau C and Kozhuharov C (2012): in V Shevelko and H Tawara (editors), Atomic Processes in Basic and Applied Physics, volume 68 of Springer series on Atomic, Optical, and Plasma Physics, Springer

Brandau C, Kozhuharov C, Müller A et al. (2003): Phys. Rev. Lett. 91, 073202

Brandau C, Kozhuharov C, Harman Z et al. (2008): Phys. Rev. Lett. 100, 073201

Brandau C, Kozhuharov C, Müller A et al. (2009): J. Phys.: Conf. Ser. 194, 012023

- (2010): Hyperfine Interact. 196, 115

Brandau C, Kozhuharov C, Müller A et al. (2013): Phys. Scr. T156, 014050

Brandau C, Kozhuharov C, Lestinsky M et al. (2015): Phys. Scr. T166, 014022

Bray I (2002): Phys. Rev. Lett. 89, 273201

Bray I, Fursa DV, Kadyrov AS, and Stelbovics AT (2010): Phys. Rev. A 81, 062704

Bray I, Fursa DV, Kadyrov AS et al. (2012): Phys. Reports 520, 135

Chatterjee S, Beyer HF, Liesen D et al. (2006): Nucl. Instrum. Methods B 245, 67

Chen MH (1992): Recombination of atomic ions, 61ff, Plenum Press, New York

Chen MH and Reed KJ (1993): Phys. Rev. A 48, 1129

Cheng KT, Chen MH, and Johnson WR (2008): Phys. Rev. A 77, 052504

Coc A, Porquet MG, and Nowacki F (2000): Phys. Rev. C 61, 015801

Colgan J and Pindzola MS (2012): Phys. Rev. Lett. 108, 053001

Colgan J, Pindzola MS, Mitnik DM, Griffin DC, and Bray I (2001): Phys. Rev. Lett. 87, 213201

Costa Fraga RA, Kalinin A, Kühnel M et al. (2012): Rev. Sci. Instrum. 83, 025102

Covington AM, Aguilar A, Covington IR et al. (2002): Phys. Rev. A 66, 062710

Danared H (1993): Nucl. Instrum. Methods A 335, 397

Danared H, Andler G, Bagge L et al. (1994): Phys. Rev. Lett. 72, 3775

Danared H, Andler G, Björkhage M et al. (2011): LSR - Low-energy Storage Ring, Technical design report, Manne-Siegbahn Laboratory, Physics Department, Stockholm University, http: //www.fair-center.eu/fileadmin/fair/publications_exp/DesignReportLSR_1.3.pdf, version 1.3, May 6th 2011

Datz S, Hippler R, Andersen LH et al. (1990): Phys. Rev. A 41, 3559

Dürr M, Dorn A, Ullrich J et al. (2007): Phys. Rev. Lett. 98, 193201

Egelhof P (1997): in Proc. of the Int. Workshop on Research with Fission Fragments, Benediktbeuren 1996, 178, World Scientific, ISBN 981-02-3140-7

Egelhof P and Kraft-Bermuth S (2005): Cryogenic Particle Detection, volume 99 of Topics in Applied 
Physics, textbook Heavy ion physics, 469, Springer-Verlag Berlin Heidelberg

Egelhof P, Beyer HF, McCammon D et al. (1996): Nucl. Instrum. Methods A 370, 263

Egelhof P, Kisselev O, Münzenberg G, Neumaier SR, and Weick H (2003): Phys. Scr. T 104, 151

Egelhof P, Bagchi S, Bönig S et al. (2015): JPS Conf. Proc. 6, 020049

Egodapitiya KN, Sharma S, Hasan A et al. (2011): Phys. Rev. Lett. 106, 153202

Ehresmann A, Klumpp S, Werner L et al. (2004a): J. Phys. B 37, L251

Ehresmann A, Liebel H, Schmoranzer H et al. (2004b): J. Phys. B 37, 389

Ehresmann A, Werner L, Klumpp S et al. (2006): J. Phys. B 39, 283

Ehrhardt H, Hesselbacher KH, Jung K et al. (1971): Z. Phys. 244, 254

Ehrhardt H, Jung K, Knoth G, and Schlemmer P (1986): Z. Phys. D 1, 3

Eichler J and Stöhlker T (2007): Phys. Reports 439, 1

Emmanouilidou A, Wang P, and Rost JM (2008): Phys. Rev. Lett. 100, 063002

Endt PM (1990): Nuc. Phys. A 521, 1

Endt PM and Firestone RB (1998): Nuc. Phys. A 633, 1

Enss C (editor) (2005): Cryogenic Particle Detection, volume 99 of Topics in Applied Physics, SpringerVerlag, Berlin

EXL (2006): Technical design report, EXL collaboration, http://www.rug.nl/kvi/research/hnp/research/ ex1/Tech_Proposal_EXL_update_080106.pdf

FAIR (2006): Experiment Proposals on Nuclear Structure and Astro Physics (NUSTAR), FAIR Baseline Technical Report 4, GSI, Darmstadt, http://www.fair-center.de/fileadmin/fair/publications_FAIR/ FAIR_BTR_4.pdf

Feist J, Nagele S, Pazourek R et al. (2008): Phys. Rev. A 77, 043420

Ferro F, Surzhykov A, and Stöhlker T (2011): Phys. Rev. A 83, 052518

Fiol J and Olson RE (2004): J. Phys. B 37, 3947

Firestone RB (1996): Table of Isotopes, Eighth Edition, Wiley-Intersciences

Fischer D, Feuerstein B, DuBois RD et al. (2002): J. Phys. B 35, 1369

Fischer D, Moshammer R, Dorn A et al. (2003a): Phys. Rev. Lett. 90, 243201

Fischer D, Moshammer R, Schulz M, Voitkiv A, and Ullrich J (2003b): J. Phys. B 36, 3555

Fischer D, Globig D, Goullon J et al. (2012): Phys. Rev. Lett. 109, 113202

Franzke B (1987): Nucl. Instrum. Methods B 24/25, 18

Freeman BM, Wrede C, Delbridge BG et al. (2011): Phys. Rev. C 83, 048801

Fritzsche S, Indelicato P, and Stöhlker T (2005): J. Phys. B 38, S707

Fritzsche S, Surzhykov A, and Stöhlker T (2009): Phys. Rev. Lett. 103, 113001

Fritzsche S, Surzhykov A, Gumberidze A, and Stöhlker T (2012): New J. Phys. 14, 083018

Fritzsche S, Surzhykov A, and Volotka A (2015): New J. Phys. 17, 103009

Gao H, DeWitt DR, Schuch R et al. (1995): Phys. Rev. Lett. 75, 4381

Geissel H, Armbruster P, Behr K et al. (1992): Nucl. Instrum. Methods B 70, 286

Geithner R, Neubert R, Vodel W et al. (2011): Rev. Sci. Instrum. 82, 013302

Genevey J, Pinston JA, Faust HR et al. (2003): Phys. Rev. C 67, 054312

Geyer S and Kester O (2011): in XXVII International Conference on Photonic, Electronic and Atomic

Collisions, Belfast, UK, 27.7.-2.8.2011, poster

Geyer S, Meusel O, and Kester O (2013): Phys. Scr. T156, 014093

Gharaibeh MF, Bizau JM, Cubaynes D et al. (2011): J. Phys. B 44, 175208 
Glorius J, Sonnabend K, Görres J et al. (2014): Phys. Rev. C 89, 065808

Goldanskii V and Namiot V (1976): Phys. Lett. B 62, 393

Goldhaber M, Grodzins L, and Sunyar AW (1958): Phys. Rev. 109, 1015

Gorda O, Braeuning-Demian A, Danared H et al. (2015): Phys. Scr. 2015, 014043

Grawe H, Löhle U, and König R (1974): Z. Phys. 268, 419

Greiner W, Müller B, and Rafelski J (1985): Quantum Electrodynamics of Strong Fields, Springer Berlin Heidelberg, ISBN 3-540-13404-2

Grieser M, Litvinov YA, Raabe R, and et al (2012): Eur. Phys. J. Special Topics 207, 1

Guan X, Bartschat K, and Schneider BI (2008): Phys. Rev. A 77, 043421

Gudmundsson M, Fischer D, Misra D et al. (2011): J. Phys. B 44, 175201

Gumberidze A, Stöhlker T, Banaś D et al. (2004): Phys. Rev. Lett. 92, 203004

- (2005): Phys. Rev. Lett. 94, 223001

Gumberidze A, Fritzsche S, Bosch F et al. (2010): Phys. Rev. A 82, 052712

Gwinner G, Hoffknecht A, Bartsch T et al. (2000): Phys. Rev. Lett. 84, 4822

Hädrich S, Klenke A, Rothhardt J et al. (2014): Nature Photonics 8, 779

Hagmann S, Stöhlker T, Kozhuharov C et al. (2010): in FD McDaniel and BL Doyle (editors), Application of Accelerators in Research and Industry: Twenty-First International Conference, 115-118, AIP Conference Proceedings, Fort Worth, Texas, USA

Hagmann $\mathrm{S}$ et al. (): To be published

Hannen V, Anielski D, Geppert C et al. (2013): J. Inst. 8, P09018

Hengstler D, Schötz C, Krantz M et al. (2013): maXs - Cryogenic Micro-Calorimeter Arrays for High Resolution X-ray Spectroscopy Experiments at FAIR, Technical design report, Kirchhoff-Institut für Physik, Universität Heidelberg, Im Neuenheimer Feld 227, D-69120 Heidelberg, http://www. fair-center.de/fileadmin/fair/publications_exp/TDR_maXs_public_2016_02_11.pdf

Hengstler D, Keller M, Schötz C et al. (2015): Phys. Scr. 2015, 014054

Henning W (1997): Nuc. Phys. A 626, 225

Herfurth F, Andelkovic Z, Barth W et al. (2015): Phys. Scr. 2015, 014065

Hillenbrand PM, Hagmann S, Atanasov D et al. (2014a): Phys. Rev. A 90, 022707

Hillenbrand PM, Hagmann S, Voitkiv AB et al. (2014b): Phys. Rev. A 90, 042713

Hillenbrand PM, Hagmann S, Jakubassa-Amundsen DH et al. (2015): Phys. Rev. A 91, 022705

Hillier J and Baker RF (1944): J. Appl. Phys. 15, 663

Hoffknecht A, Uwira O, Schennach S et al. (1998): J. Phys. B 31, 2415

Hörndl M, Yoshida S, Wolf A, Gwinner G, and Burgdörfer J (2005): Phys. Rev. Lett. 95, 243201

Hörndl M, Yoshida S, Wolf A et al. (2006): Phys. Rev. A 74, 052712

Howard WM, Meyer BS, and Woosley SE (1991): Astrophys. J. 373, L5

Hu SX and Collins LA (2004): Phys. Rev. A 70, 013407

Hubele R, LaForge A, Schulz M et al. (2013): Phys. Rev. Lett. 110, 133201

Hubele R, Schuricke M, Goullon J et al. (2015): Rev. Sci. Instrum. 86, 033105

Hubricht G and Träbert E (1987): Z. Phys. D 7, 243

Iliadis C (1997): Nuc. Phys. A 618, 166

Iliadis C, D’Auria JM, Starrfield S, Thompson WJ, and Wiescher M (2001): Astrophys. J. Suppl. Ser. 134, 151

Ilieva S, Kiselev O, Emling H et al. (2007): Eur. Phys. J. Spec. Top. 150, 357 
ILIMA (2006): Technical proposal, ILIMA collaboration, http://www.fair-center.eu/fileadmin/fair/ experiments/NUSTAR/Pdf/ILIMA_tp_20050120-printed.pdf

Jakubassa-Amundsen DH (2003): J. Phys. B 36, 1971

- (2007): Eur. Phys. J. D 41, 267

Jeet J, Schneider C, Sullivan ST et al. (2015): Phys. Rev. Lett. 114, 253001

Jentschura UD (2015): Phys. Rev. A 92, 012123

Jia CC, Lahmam-Bennani A, Duguet A et al. (2002): J. Phys. B 35, 1103

Johnson W (2011): Can. J. Phys. 89, 429

José J and Hernanz M (1998): Astrophys. J. 494, 680

José J, Coc A, and Hernandez M (2001): Astrophys. J. 560, 897

José J, Hernanz M, Amari S, Lodders K, and Zinner E (2004): Astrophys. J. 612, 414

José J, Hernanz M, and Iliadis C (2006): Nuc. Phys. A 777, 550

Jung M, Bosch F, Beckert K et al. (1992): Phys. Rev. Lett. 69, 2164

Karshenboim SG (2015): Phys. Rev. A 91, 012515

Katayama T, Kamerdzhiev V, Lehrach A et al. (2015): Phys. Scr. T166, 014073

Kay B, Freeman S, Schiffer J et al. (2008): Phys. Lett. B 658, 216

Keller S and Dreizler RM (1998): Phys. Rev. A 57, 3652

Keller S, Dreizler RM, Ancarani LU et al. (1999): Phys. Rev. A 59, 1284

Kienle P, Bosch F, Bühler P et al. (2013): Phys. Lett. B 726, 638

Kieslich S, Schippers S, Shi W et al. (2004): Phys. Rev. A 70, 042714

Kishimoto S, Yoda Y, Seto M et al. (2000): Phys. Rev. Lett. 85, 1831

Kishimoto S, Yoda Y, Kobayashi Y et al. (2006): Phys. Rev. C 74, 031301

Kjeldsen H, Folkmann F, Knudsen H et al. (1999a): J. Phys. B 32, 4457

- (1999b): Astrophys. J. 524, L143

Kjeldsen H, Hansen JE, Folkmann F et al. (2001): Astrophys. J. Suppl. Ser. 135, 285

Klaft I, Borneis S, Engel T et al. (1994): Phys. Rev. Lett. 73, 2425

Kluge HJ, Beier T, Blaum K et al. (2008): in S Salomonson and E Lindroth (editors), Current Trends in Atomic Physics, volume 53 of Advances in Quantum Chemistry, 83-98, Academic Press

Koenig M, Benuzzi-Mounaix A, Ravasio A et al. (2005): Plasma Physics and Controlled Fusion 47, B441

Kollmus H, Moshammer R, Olson RE et al. (2002): Phys. Rev. Lett. 88, 103202

Kozhedub Y, Andreev OV, Shabaev VM et al. (2008): Phys. Rev. A 77, 032501

Kraft-Bermuth S, Andrianov V, Bleile A et al. (2012): J. Low Temp. Phys. 167, 765

Kraft-Bermuth S, Egelhof P, Echler A et al. (2014): The SiM-X Project: Silicon Microcalorimeters for high-precision X-ray spectroscopy, Technical design report, FAIR, Darmstadt, http://www. fair-center.de/fileadmin/fair/publications_exp/TDR_siliconmicrocalorimeters_public.pdf

Kraft-Bermuth S, Andrianov V, Bleile A et al. (2015): Phys. Scr. T166, 014028

Krieger A, Geppert C, Catherall R et al. (2011): Nucl. Instrum. Methods A 632, 23

Krieger A, Blaum K, Bissell ML et al. (2012): Phys. Rev. Lett. 108, 142501

Kusakabe M, Iwamoto N, and Nomoto K (2011): Astrophys. J. 726, 25

Labzowsky LN, Nefiodov AV, Plunien G et al. (2001): Phys. Rev. A 63, 054105

Lada CJ (2006): Astrophys. J. 640, L63

LaForge A, Hubele R, Goullon J et al. (2013): J. Phys. B 46, 031001 
Lahmam-Bennani A, Staicu Casagrande EM, Naja A, Dal Cappello C, and Bolognesi P (2010): J. Phys. $B$ 43, 105201

Lahmam-Bennani A et al. (2002): in R Dreizler et al. (editors), New Directions in Atomic Physics, Springer,

Lambert DL (1992): Astron. Astrophys. Rev. 3, 201

Lamour E, Prigent C, Reuschl R et al. (2010): Fast ion-slow ion collisions - FISIC project, Letter of intent, SPIRAL2, http://pro.ganil-spiral2.eu/spiral2/instrumentation/s3/working-documents/ loi-day-1-experiments/fast-ion-slow-ion-collisions-2013fisic-project/view

Leising MD and Clayton DD (1987): Astrophys. J. 323, 159

Lestinsky M (2007): High-Resolution Electron Collision Spectroscopy with Multicharged Ions in Merged Beams, Dissertation, Universität Heidelberg, http://www.ub.uni-heidelberg.de/archiv/7334/

Lestinsky M, Lindroth E, Orlov DA et al. (2008): Phys. Rev. Lett. 100, 033001

Lestinsky M, Angert N, Bär R et al. (2012): CRYRING@ESR: A study group report, Project study, GSI, Darmstadt, https://www.gsi.de/fileadmin/SPARC/documents/Cryring/ReportCryring_40ESR.PDF

Lestinsky M, Bräuning-Demian A, Danared H et al. (2015): Phys. Scr. T166, 014075

Levy C, Baartman R, Behr J et al. (2004): Nuc. Phys. A 746, 206

Li J and Dong C (2010): Plasma Science and Technology 12, 364

Lindroth E, Danared H, Glans P et al. (2001): Phys. Rev. Lett. 86, 5027

Linkemann J, Kenntner J, Müller A et al. (1995): Nucl. Instrum. Methods B 98, 154

Litvinov Y, Bosch F, Winckler N et al. (2008): Phys. Lett. B 664, 162

Lochmann M, Jöhren R, Geppert C et al. (2014): Phys. Rev. A 90, 030501

Mannervik S (2003): Phys. Scr. T 105, 67

Marrs RE, Elliott SR, and Stöhlker T (1995): Phys. Rev. A 52, 3577

Märtin R, Weber G, Barday R et al. (2012): Phys. Rev. Lett. 108, 264801

Matveev A, Parthey CG, Predehl K et al. (2013): Phys. Rev. Lett. 110, 230801

McCarthy IE (1995): Aust. J. Phys. 48, 1 http://www.publish.csiro.au/paper/PH950001.htm

McCarthy IE and Weigold E (1991): Reports on Progress in Physics 54, 789

McGovern M, Assafrão D, Mohallem JR, Whelan CT, and Walters HRJ (2010): Phys. Rev. A 81, 042704

McGuire JH (1982): Phys. Rev. Lett. 49, 1153

- (1997): Electron Correlation Dynamics in Atomic Collisions, Cambridge University Press

Mei B, Aumann T, Bishop S et al. (2015): Phys. Rev. C 92, 035803

Misra D, Schmidt HT, Gudmundsson M et al. (2009): Phys. Rev. Lett. 102, 153201

Moeini H, Ilieva S, Aksouh F et al. (2011): Nucl. Instrum. Methods A 634, 77

Mohr PJ, Taylor BN, and Newell DB (2008): Rev. Mod. Phys. 80, 633

Möller G, Träbert E, Lodwig V et al. (1989): Z. Phys. D 11, 333

Moshammer R, Fainstein PD, Schulz M et al. (1999): Phys. Rev. Lett. 83, 4721

Müller A (1995): Nucl. Fusion Suppl. 6, 59 http://www-pub.iaea.org/MTCD/Publications/PDF/ Pub23vol6_web.pdf

Müller A (2008): Adv. At. Mol. Opt. Phys. 55, 293

Müller RA, Yerokhin VA, and Surzhykov A (2014): Phys. Rev. A 90, 032707

Mutterer M, Egelhof P, Eremin V et al. (2015): Phys. Scr. T166, 014053

Niering M, Holzwarth R, Reichert J et al. (2000): Phys. Rev. Lett. 84, 5496

NNDC (2012): http://www.nndc.bnl.gov 
Nofal M, Hagmann S, Stöhlker T et al. (2007): Phys. Rev. Lett. 99, 163201

Nörtershäuser W (2011): Hyperfine Interact. 199, 131

Nörtershäuser W and Sanchez R (2015): Phys. Scr. T166, 014020

Nörtershäuser W, Tiedemann D, Žáková M et al. (2009): Phys. Rev. Lett. 102, 062503

Novotny C, Huber G, Karpuk S et al. (2009): Phys. Rev. A 80, 022107

Nörtershäuser W, Lochmann M, Jöhren R et al. (2013): Phys. Scr. T156, 014016

Orban I, Loch SD, Böhm S, and Schuch R (2010): Astrophys. J. 721, 1603

Orlov DA, Sprenger F, Lestinsky M et al. (2005): J. Phys.: Conf. Ser. 4, 290

Palffy A (2010): Contemp. Phys. 51, 471

Pálffy A, Scheid W, and Harman Z (2006): Phys. Rev. A 73, 012715

Pálffy A, Evers J, and Keitel CH (2007): Phys. Rev. Lett. 99, 172502

Pálffy A, Harman Z, Kozhuharov C et al. (2008): Phys. Lett. B 661, 330

Parikh A, Faestermann T, Hertenberger R et al. (2009): Phys. Rev. C 80, 015802

Parthey CG, Matveev A, Alnis J et al. (2011): Phys. Rev. Lett. 107, 203001

Peik E and Okhapkin M (2015): C. R. Physique 16, 516

Pies C, Schäfer S, Heuser S et al. (2012): J. Low Temp. Phys. 167, 269

Pindzola MS, Badnell NR, and Griffin DC (1992): Phys. Rev. A 45, R7659

Pohl R, Antognini A, Nez F et al. (2010): Nature 466, 213

Pohl R, Gilman R, Miller GA, and Pachucki K (2013): Annu. Rev. Nucl. Part. Sci. 63, 175

Prozorov A, Labzowsky L, Liesen D, and Bosch F (2003): Phys. Lett. B 574, 180

Rauscher T and Thielemann FK (2000): At. Data Nucl. Data Tables 75, 1

- (2004): At. Data Nucl. Data Tables 88, 1

Reed KJ, Chen MH, and Moores DL (1990): Phys. Rev. A 42, 5315

— (1991): Phys. Rev. A 44, 4336

Reifarth R, Lederer C, and Käppeler F (2014): J. Phys. G 41, 053101

Reinhardt S, Saathoff G, Karpuk S et al. (2006): Optics Communications 261, 282

Reinhardt S, Bernhardt B, Geppert C et al. (2007): Optics Communications 274, 354

Ren X, Senftleben A, Pflüger T et al. (2010): Phys. Rev. A 82, 032712

Ren X, Bray I, Fursa D et al. (2011a): Phys. Rev. A 83, 052711

Ren X, Senftleben A, Pflüger T et al. (2011b): Phys. Rev. A 83, 052714

Ren X, Pflüger T, Ullrich J et al. (2012): Phys. Rev. A 85, 032702

Rescigno TN, Baertschy M, Isaacs WA, and McCurdy CW (1999): Science 286, 2474

Reuschl R, Gumberidze A, Stöhlker T et al. (2006): Radiation Physics and Chemistry 75, 1740

Reuschl R, Gumberidze A, Kozhuharov C et al. (2008): Phys. Rev. A 77, 032701

Rinn K, Müller A, Eichenauer H, and Salzborn E (1982): Rev. Sci. Instrum. 53, 829

Rothhardt J, Hädrich S, Demmler S et al. (2014): Phys. Rev. Lett. 112, 233002

Rothhardt J et al. (2015): in SPARC Topical Workshop, Fodele, Crete, Greece, September 22-28

Rzadkiewicz J, Stöhlker T, Banas D et al. (2006): Phys. Rev. A 74, 012511

Saghiri AA, Linkemann J, Schmitt M et al. (1999): Phys. Rev. A 60, R3350

Sanchez R, Ullmann J, Vollbrecht J et al. (2015): Phys. Scr. T166, 014021

Saumon D and Guillot T (2004): Astrophys. J. 609, 1170

Savin DW, Bartsch T, Chen MH et al. (1997): Astrophys. J. 489, L115

Schatz H, Aprahamian A, Görres J et al. (1998): Phys. Reports 294, 167 
Scheidenberger C, Beckert K, Beller P et al. (2006): Hyperfine Interact. 173, 61

Schiffer JP, Freeman SJ, Caggiano JA et al. (2004): Phys. Rev. Lett. 92, 162501

Schippers S (2015): Nucl. Instrum. Methods B 350, 61

Schippers S, Bartsch T, Brandau C et al. (1998): J. Phys. B 31, $4873-4886$

Schippers S, Müller A, Gwinner G et al. (2001): Astrophys. J. 555, 1027

Schippers S, Gwinner G, Brandau C et al. (2005): Nucl. Instrum. Methods B 235, 265

Schippers S, Schmidt EW, Bernhardt D et al. (2007): Phys. Rev. Lett. 98, 033001

Schippers S, Bernhardt D, Müller A et al. (2011): Phys. Rev. A 83, 012711

Schippers S, Bernhardt D, Müller A et al. (2012): Phys. Rev. A 85, 012513

Schippers S, Ricz S, Buhr T et al. (2014): J. Phys. B 47, 115602

Schippers S, Kilcoyne ALD, Phaneuf RA, and Müller A (2015): Contemp. Phys. 57, 215

von Schmid M, Bagchi S, Bönig S et al. (2014): EPJ Web of Conferences 66, 03093

von Schmid M, Bagchi S, Bönig S et al. (2015): Phys. Scr. T166, 014005

Schmidt H, Cederquist H, Schuch R et al. (1997): Hyperfine Interact. 108, 339

Schmidt HT, Forck P, Grieser M et al. (1994): Phys. Rev. Lett. 72, 1616

Schmidt HT, Fardi A, Schuch R et al. (2002): Phys. Rev. Lett. 89, 163201

Schmidt HT, Fischer D, Berenyi Z et al. (2008): Phys. Rev. Lett. 101, 083201

Schramm U, Berger J, Grieser M et al. (1991): Phys. Rev. Lett. 67, 22

Schubert E, Schuck A, Jung K, and Geltman S (1979): J. Phys. B 12, 967

Schuch R, Lindroth E, Madzunkov S et al. (2005): Phys. Rev. Lett. 95, 183003

Schulz M, Moshammer R, Gerchikov LG, Sheinerman SA, and Ullrich J (2001): J. Phys. B 34, L795

Schulz M, Moshammer R, Fischer D et al. (2003): Nature 422, 48

Schulz M, Dürr M, Najjari B, Moshammer R, and Ullrich J (2007): Phys. Rev. A 76, 032712

Seelig P, Borneis S, Dax A et al. (1998): Phys. Rev. Lett. 81, 4824

Sepp WD, Fricke B, and Morović T (1981): Phys. Lett. 81A, 258

Shi W, Böhm S, Böhme C et al. (2001): Eur. Phys. J. D 15, 145

Shi W, Bartsch T, Böhme C et al. (2002): Phys. Rev. A 66, 022718

Shi W, Jacobi J, Knopp H, Schippers S, and Müller A (2003): Nucl. Instrum. Methods B 205, 201

Soff G, Beier T, Greiner M, Persson H, and Plunien G (1998): Adv. Quant. Chem. 30, 125

SPARC (2005): Technical Proposal, https://www.gsi.de/fileadmin/SPARC/documents/ sparc-technical-proposal_print.pdf

Spies W, Uwira O, Müller A et al. (1995): Nucl. Instrum. Methods B 98, 158

Spruck K, Badnell NR, Krantz C et al. (2014): Phys. Rev. A 90, 032715

Stahle C, Kelley RL, McCammon D, Moseley SH, and Szymkowiak AE (1996): Nucl. Instrum. Methods A 370, 173

Starrfield S, Truran JW, Sparks WM, and Kitter GS (1972): Astrophys. J. 176, 169

Steck M, Brandau C, Dimopoulou C, Kozhuharov C, and Nolden F (2010): in Proceedings of the 1st Int. Part. Acc. Conf. (IPAC10), Kyoto, Japan, May 23-28, 840ff, http://accelconf.web.cern.ch/ AccelConf/IPAC10/papers/mopd066.pdf

Stiebing KE, Alexandrov V, Dörner R et al. (2010): Nucl. Instrum. Methods A 614, 10

Støchkel K, Eidem O, Cederquist H et al. (2005): Phys. Rev. A 72, 050703

Stöhlker T, Mokler PH, Beckert K et al. (1993): Phys. Rev. Lett. 71, 2184

Stöhlker T, Kozhuharov C, Mokler PH et al. (1995): Phys. Rev. A 51, 2098 
Stöhlker T, Bosch F, Gallus A et al. (1997): Phys. Rev. Lett. 79, 3270

Stöhlker T, Ludziejewski T, Reich H et al. (1998): Phys. Rev. A 58, 2043

Stöhlker T, Ludziejewski T, Bosch F et al. (1999): Phys. Rev. Lett. 82, 3232

Stöhlker T, Mokler PH, Bosch F et al. (2000): Phys. Rev. Lett. 85, 3109

Stöhlker T, Ma X, Ludziejewski T et al. (2001): Phys. Rev. Lett. 86, 983

Stöhlker T, Gumberidze A, Trassinelli M et al. (2008): Lecture Notes in Physics 745, 157

Stöhlker T, Litvinov YA, Bräuning-Demian A et al. (2014): Hyperfine Interact. 227, 45

Stöhlker T, Bagnoud V, Blaum K et al. (2015): Nucl. Instrum. Methods B 365, 680

Streicher B, Egelhof P, Ilieva S et al. (2011): Nucl. Instrum. Methods A 654, 604

Surzhykov A, , Litvinov YA, Fritzsche S, and Stöhlker T (2013): Phys. Rev. A 87, 052507

Tashenov S, Stöhlker T, Banaś D et al. (2006): Phys. Rev. Lett. 97, 223202

Tashenov S, Banaś D, Beyer H et al. (2014): Phys. Rev. Lett. 113, 113001

Thümmler T, Marx R, and Weinheimer C (2009): New J. Phys. 11, 103007

Tkalya EV, Schneider C, Jeet J, and Hudson ER (2015): Phys. Rev. C 92, 054324

Toleikis S, Manil B, Berdermann E et al. (2004): Phys. Rev. A 69, 022507

Träbert E (2000): Phys. Scr. 61, 257

- (2002): Can. J. Phys. 80, 1481

Träbert E, Doerfert J, Granzow J et al. (1994): Phys. Lett. A 188, 355

- (1995): Phys. Lett. A 202, 91

Träbert E, Gwinner G, Knystautas EJ, Tordoir X, and Wolf A (1999): J. Phys. B 32, L491

Trassinelli M, Kumar A, Beyer HF et al. (2009): Europhys. Lett. 87, 63001

Travaglio C, Röpke FK, Gallino R, and Hillebrandt W (2011): Astrophys. J. 739, 93

Trotsenko S, Kumar A, Volotka AV et al. (2010): Phys. Rev. Lett. 104, 033001

Träbert E (2014): Phys. Scr. 89, 114003

Tupitsyn II, Kozhedub YS, Shabaev VM et al. (2010): Phys. Rev. A 82, 042701

— (2012): Phys. Rev. A 85, 032712

Ullmann J, Andelkovic Z, Dax A et al. (2015): J. Phys. B 48, 144022

Ullrich J, Hagmann S, Moshammer R et al. (1996): Electron spectroscopy and (e, 2e) experiments at the new internal esr-electron target, Letter of Intent 17, GSI-ESR

Ullrich J, Moshammer R, Dorn A et al. (2003): Rep. Prog. Phys. 66, 1463

Uwira O, Müller A, Linkemann J et al. (1997a): Hyperfine Interact. 108, 149

Uwira O et al. (1997b): Hyperfine Interact. 108, 167

Vigren E, Hamberg M, Zhaunerchyk V et al. (2009): Astrophys. J. 695, 317

Vigren E, Hamberg M, Zhaunerchyk V et al. (2010): Astrophys. J. 709, 1429

Walker P, Litvinov YA, and Geissel H (2013): Int. J. Mass Spectrom. 349-350, 247

Wanajo S, Hashimoto M, and Nomoto K (1999): Astrophys. J. 523, 409

Wang X, Schneider K, LaForge A et al. (2012): J. Phys. B 45, 211001

Weber G, Bräuning H, Surzhykov A et al. (2010): Phys. Rev. Lett. 105, 243002

Widmann E (2015): Phys. Scr. T166, 014074

Wu ZW, Surzhykov A, and Fritzsche S (2014): Phys. Rev. A 89, 022513

- (2015): Phys. Rev. A 91, 056502

Xia J, Zhan W, Wei B et al. (2002): Nucl. Instrum. Methods A 488, 11

Yamaoka H, Oura M, Kawatsura K et al. (2001): Phys. Rev. A 65, 012709 
Yerokhin VA and Shabaev VM (2015): J. Phys. Chem. Ref. Data 44, 033103

Yerokhin VA, Surzhykov A, and Fritzsche S (2014): Phys. Rev. A 90, 022509

Yue K, Bagchi S, Diebold S et al. (2011): in 8th International Conference on Nuclear Physics at Storage Rings (Stori11), Laboratori Nazionali di Frascati dell'INFN, Italy, October 9-14 2011, 014, http: //pos.sissa.it/archive/conferences/150/014/STORI11_014.pdf

Zamora JC, Bagchi S, Bönig S et al. (2015): Phys. Scr. T166, 014006

Zhong Q, Aumann T, Bishop S et al. (2010): J. Phys.: Conf. Ser. 202, 012011

Zolotorev M and Budker D (1997): Phys. Rev. Lett. 78, 4717

Zou Y, Hutton R, Feili D et al. (2005): Nucl. Instrum. Methods B 235, 192

Zubova NA, Kozhedub YS, Shabaev VM et al. (2014): Phys. Rev. A 90, 062512 